\title{
Propagation Measurement Based Estimation and Comparison of DSSS Microcellular Mobile Link Performance at 2.25 and $5.8 \mathrm{GHz}$
}

\author{
By \\ Min Han \\ A thesis submitted to \\ the Faculty of Graduate Studies and Research \\ in partial fulfilment of requirement for the degree of

\section{Master of Applied Science}
Ottawa-Carleton Institute for Electrical and Computer Engineering Department of Systems and Computer Engineering
Carleton University
Ottawa, Ontario, Canada

\author{
November 2006 \\ (C) Copyright \\ 2006, Min Han
}




$\begin{array}{ll}\begin{array}{l}\text { Library and } \\ \text { Archives Canada }\end{array} & \begin{array}{l}\text { Bibliothèque et } \\ \text { Archives Canada }\end{array} \\ \begin{array}{l}\text { Published Heritage } \\ \text { Branch }\end{array} & \begin{array}{l}\text { Direction du } \\ \text { Patrimoine de l'édition }\end{array} \\ \begin{array}{l}\text { 395 Wellington Street } \\ \text { Ottawa ON K1A ON4 }\end{array} & \begin{array}{l}\text { 395, rue Wellington } \\ \text { Ottawa ON K1A ON4 } \\ \text { Canada }\end{array}\end{array}$

Your file Votre référence ISBN: 978-0-494-23339-9 Our file Notre référence ISBN: 978-0-494-23339-9

NOTICE:

The author has granted a nonexclusive license allowing Library and Archives Canada to reproduce, publish, archive, preserve, conserve, communicate to the public by telecommunication or on the Internet, loan, distribute and sell theses worldwide, for commercial or noncommercial purposes, in microform, paper, electronic and/or any other formats.

The author retains copyright ownership and moral rights in this thesis. Neither the thesis nor substantial extracts from it may be printed or otherwise reproduced without the author's permission.
AVIS:

L'auteur a accordé une licence non exclusive permettant à la Bibliothèque et Archives Canada de reproduire, publier, archiver, sauvegarder, conserver, transmettre au public par télécommunication ou par l'Internet, prêter, distribuer et vendre des thèses partout dans le monde, à des fins commerciales ou autres, sur support microforme, papier, électronique et/ou autres formats.

L'auteur conserve la propriété du droit d'auteur et des droits moraux qui protège cette thèse. $\mathrm{Ni}$ la thèse ni des extraits substantiels de celle-ci ne doivent être imprimés ou autrement reproduits sans son autorisation.
In compliance with the Canadian

Privacy Act some supporting forms may have been removed from this thesis.

While these forms may be included in the document page count, their removal does not represent any loss of content from the thesis.
Conformément à la loi canadienne sur la protection de la vie privée, quelques formulaires secondaires ont été enlevés de cette thèse.

Bien que ces formulaires aient inclus dans la pagination, il n'y aura aucun contenu manquant.

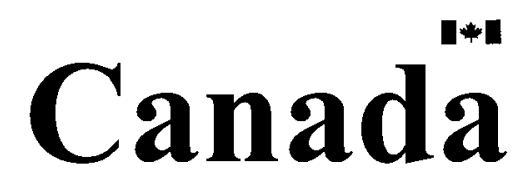




\section{Abstract}

As a result of increasing demand for greater mobile system capacities, expansion into frequency bands other than the current IMT2000 band near $2 \mathrm{GHz}$ is being considered. Estimation of expected mobile link performance at other frequencies is thus important.

During this thesis project, quasi-simultaneous propagation measurements at 2 and 6 $\mathrm{GHz}$ frequencies were conducted in downtown Ottawa. Measurement-based analysis and comparison of time-varying channel characteristics are reported. These show that there is often a larger signal fluctuation after Rake combining at $6 \mathrm{GHz}$. Results also show that in NLOS conditions, the RMS delay spreads at $2 \mathrm{GHz}$ are slightly greater.

Measurement-based prediction of error performance resulting from the use of Rake receivers on BPSK-DSSS mobile links was conducted. For transmissions with low data rates in IMT2000 bandwidths, self-interference that results from multipath propagation is insignificant, enabling the use of well-known prediction equations. Results show that error performance would be slightly better at $2 \mathrm{GHz}$. However, channel protection in NLOS conditions would still be required in both frequency bands to achieve BER performance of $10^{-3}$ throughout 2-city block microcells. For the consideration of transmission with high data rates, the effects of self-interference can not be ignored. To accurately account for this, a recently reported semi-analytical BER prediction technique was used. Resulting comparisons of achievable performance in the two bands were similar to those for the low data rate case.

iii 


\section{Acknowledgements}

I would like to thank my Professor R. H. M. Hafez for his advising in my academic study, and for his great and continuous guidance and encouragement during the course of my thesis.

I would also like to express my deepest gratitude to my Supervisor Doctor R. J. C. Bultitude at $\mathrm{CRC}$, for his continuous and patient guidance and support, as well as his timely advice contributed greatly to the completion of this thesis.

I am very glad to thank Doctor N. Adnani at CRC for his time, his ideas and valuable guidance during the thesis work.

Special thanks to Jeff Pugh and Hong Zhu at CRC for their work and support of this project.

I express my sincere gratitude to my parents, my husband and my son for their understanding and support.

iv 


\section{Table of Contents}

$\begin{array}{lr}\text { Abstract } & \text { iii } \\ \text { Acknowledgements } & \text { iv } \\ \text { Table of Contents } & \mathrm{v} \\ \text { List of Figures } & \text { vii } \\ \text { List of Tables } & \text { xii } \\ \text { List of Symbols } & \text { xii } \\ \text { List of Acronyms } & \text { xvii }\end{array}$

1. Introduction 1

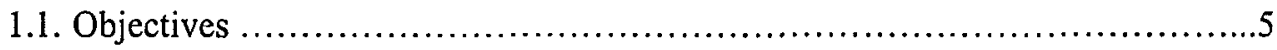

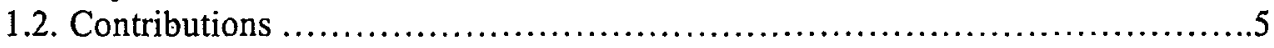

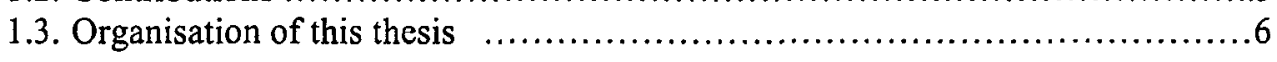

2. Background $\quad 7$

2.1. Channel characteristics .....................................................

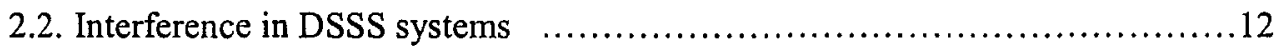

2.3. Rake receivers in a DSSS system ........................................ 13

2.4. Literature Review .................................................... 19

3. Experimental Set-up and Data Analysis 26

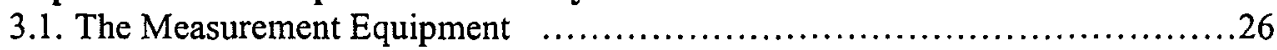

3.1.1. Description of the channel measurement system ......................27

3.1.2. System calibration tests...............................................

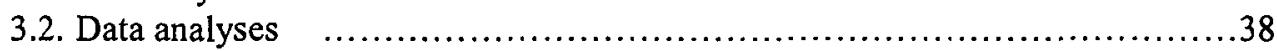

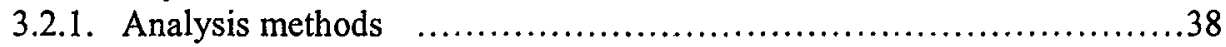

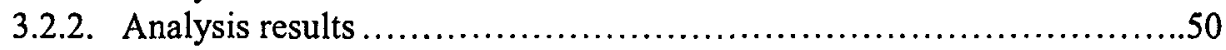

3.2.2.1. Results from analyses of the old set of data .........................50

3.2.2.2. Results from analyses of the new set of data (Tx on Kent Street, between Gloucester St. and Laurier Ave.) ..................................58

3.2.2.3. Results from analyses of the new set of data (Tx on Laurier Avenue, between Kent St. and Bank St).....................................64

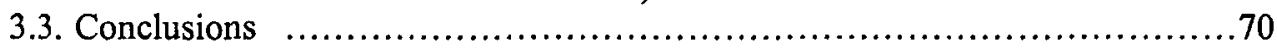


4. Semi - Analytical Prediction of BER Performance on DSSS Mobile Radio Links

4.1. BER Prediction method .....................................................71

4.2. Verification of methodology ........................................... 73

4.3. BER predictions for high data rate transmission $\ldots \ldots \ldots \ldots \ldots \ldots \ldots \ldots \ldots \ldots \ldots$

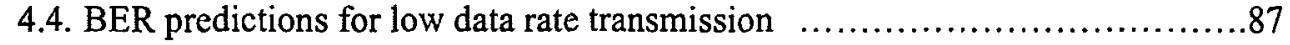

4.4.1. BER predictions from data recorded with $\mathrm{Tx}$ at site $\mathrm{Tx} 2 \ldots \ldots \ldots \ldots \ldots \ldots . . .17$

4.4.2. BER predictions from data recorded with $\mathrm{Tx}$ at site $\mathrm{Tx} 1$........................93

4.5. Conclusions............................................................ 99

5. Summary, conclusions and recommendations for further work 102

$\begin{array}{ll}\text { References } & 108\end{array}$

Appendix: Semi - Analytical Technique (SAT) review 111 


\section{List of Figures}

2.1 A diagram of a downlink radio channel in a mobile system .......................8

2.2 (a) transmitted signal with flat fading, (b) transmitted signal with frequency

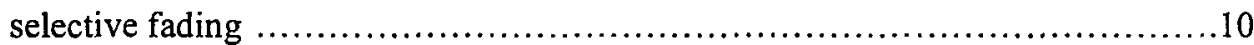

2.3 Block diagram of the Rake section in a typical DSSS receiver ....................14

2.4 Comparison between a binominal distribution and the distribution of crosscorrelation of two integer-shifted 5 -chip long sequences. ...................... 18

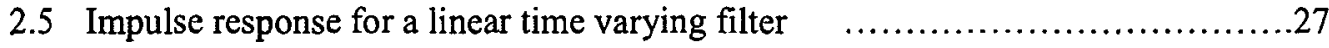

3.1.1 ACFs and TFs of $\mathrm{BB}$ reference sequences at 2 and $6 \mathrm{GHz} \ldots \ldots \ldots \ldots \ldots \ldots . .29$

3.1.2 Measurement system Transmitter block diagram $\quad$...........................32

3.1.3 Measurement system Receiver block diagram …..........................33

3.1.4 BB calibration tests configuration .......................................

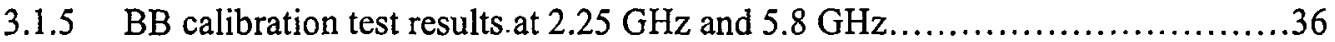

3.2.1 APDP and MPGs estimated from the old set of data on Bank Street, between Gloucester St. and Laurier Ave.............................................39

3.2.2 APDP and MPGs estimated from the old set of data on Kent Street, between

Slater St. and Albert Ave...................................................40

3.2.3 Envelope time series of the three strongest MPGs at $2 \mathrm{GHz}$ on Kent Street, between Slater St. and Albert St.

vii 
3.2.4 Envelope time series of the three strongest MPGs at $6 \mathrm{GHz}$ on Kent Street, between Slater St. and Albert St.

3.2.5 ECDFs of envelop fading for the three strongest MPGs at $2 \mathrm{GHz}$ on Kent Street, between Slater St. and Albert St.

3.2.6 ECDFs of envelop fading for the three strongest MPGs at $6 \mathrm{GHz}$ on Kent Street, between Slater St. and Albert St.

3.2.7 Comparison of Time series and ECDFs of envelop fading after Rake combining of the three strongest MPGs at $6 \mathrm{GHz}$ and those for the strongest MPG at $2 \mathrm{GHz}$ on Kent Street, between Slater St. and Albert St. . .46

3.2.8 RMS delay spread from the old set of data at $2 \mathrm{GHz}$ on Bank Street, between Gloucester St. and Laurier Ave.

3.2.9 RMS delay spread estimated from the new set of data recorded on Kent Street, between Laurier Ave. and Slater St. with Tx on Laurier Ave.

3.2.10 Map showing measurement locations for the old set of data, recorded with Tx on Laurier Avenue, between Kent St. and Bank St.

3.2.11 ECDFs of fading ranges for the three strongest MPGs from 27 street sections in downtown Ottawa .53

3.2.12 ECDFs and scatter plot of fading ranges after Rake combining for the MPGs with relative power greater than $-7 \mathrm{~dB}$ for the old set of data at 2 and $6 \mathrm{GHz}$ with $\mathrm{Tx}$ at site $\mathrm{Tx} 1$. . .55

3.2.13 ECDFs of RMS delay spread for the old set of data at 2 and $6 \mathrm{GHz}$ in downtown Ottawa. .57

viii 
3.2.14 Map showing measurement locations for the new set of data, recorded with the Tx on Kent Street, between Gloucester St. and Laurier Ave

3.2.15 ECDFs of fading ranges for the three strongest MPGs from 48 street sections in downtown Ottawa. .60

3.2.16 ECDFs and scatter plot of fading ranges after Rake combining for the MPGs with relative power greater than $-7 \mathrm{~dB}$ for 48 street sections with $\mathrm{Tx}$ at site $\mathrm{Tx} 2 \quad \ldots 62$

3.2.17 ECDFs of RMS delay spread for 48 street sections with $\mathrm{Tx}$ at site $\mathrm{Tx} 2$ .63

3.2.18 Map showing measurement locations for the new set of data, recorded with $\mathrm{Tx}$ on Laurier Avenue, between Kent St. and Bank St.

3.2.19 ECDFs of fading ranges for the three strongest MPGs from 147 street sections in downtown Ottawa .66

3.2.20 ECDFs and scatter plot of fading ranges after Rake combining for MPGs with relative power greater than $-7 \mathrm{~dB}$ for 147 street sections with $\mathrm{Tx}$ at site $\mathrm{Tx} 1 \ldots .68$

3.2.21 ECDFs of RMS delay spread for 147 street sections with $T x$ at site Tx 1 at 2 and 6 $\mathrm{GHz}$. 69

4.2.1 Comparison of analytical BER predications with those made using the SAT for one-tap and two-tap Rayleigh fading channels

4.2.2 Comparison between BER results calculated using the SAT and BER estimated using a Gaussian model for the PDF of SI for a Rake receiver with $\mathrm{L}=1$ and two equal powers of MPGs .77

4.3.1 BER vs. Average $E_{b} / N_{o}$ results based on time series measurements on Kent Street, between Gloucester St. and Laurier Ave. with $N_{d}=7$ 
4.3.2 BER vs. Average $E_{b} / N_{o}$ results based on time series measurements on Kent Street, between Slater St. and Albert St. with $N_{d}=7$ .82

4.3.3 ECDFs of the average $E_{b} / N_{o}$ required to achieve $\mathrm{BER}=10^{-3}$ at 2 and $6 \mathrm{GHz} 83$

4.3.4 ECDFs of BER at 2 and $6 \mathrm{GHz}$ if an average $E_{b} / N_{o}$ of $15 \mathrm{~dB}$ is available on all 27 street sections. .85

4.3.5 ECDFs of BER at 2 and $6 \mathrm{GHz}$ if an average $E_{b} / N_{0}$ of $20 \mathrm{~dB}$ is available on all 27 street sections. .86

4.4.1 BER vs. Average $E_{b} / N_{o}$ based on time series measurements on Lyon Street, between Laurier Ave. and Gloucester St. with $N_{d}=128$

4.4.2 ECDFs for the average value of $E_{b} / N_{o}$ required achieving a BER of $10^{-3}$ at 2 and $6 \mathrm{GHz}$ with Tx on Kent Street

4.4.3 ECDFs of BER at 2 and $6 \mathrm{GHz}$ if an average $E_{b} / N_{o}$ of $15 \mathrm{~dB}$ is available at both frequencies with $\mathrm{Tx}$ on Kent Street.

4.4.4 ECDFs of BER at 2 and $6 \mathrm{GHz}$ if an average $E_{b} / N_{o}$ of $20 \mathrm{~dB}$ is available at both frequencies with Tx on Kent Street.

4.4.5 BER vs. Average $E_{b} / N_{o}$ results based on time series measurements on Bay

Street, between Gloucester St. and Laurier Ave. with $N_{d}=128$

4.4.6 ECDFs for the average value of $E_{b} / N_{o}$ required achieving a BER of $10^{-3}$ at 2 and $6 \mathrm{GHz}$ with $\mathrm{Tx}$ on Laurier Avenue .95

4.4.7 ECDFs of BER if an average $E_{b} / N_{o}$ of $15 \mathrm{~dB}$ is available at both frequencies with Tx on Laurier Avenue. 
4.4.8 ECDFs of BER if an average $E_{b} / N_{o}$ of $20 \mathrm{~dB}$ is available at both frequencies with Tx on Laurier Avenue...............................................98 


\section{List of Tables}

3.1 Medians of the relative powers for the first three strongest MPGs from 27 street

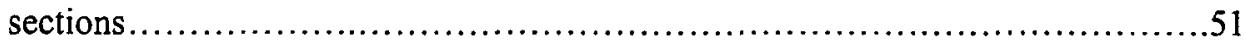

3.2 Medians of envelop fading ranges $(10 \%: 90 \%)$ for 27 street sections ..............54

3.3 Medians of relative average powers for the first three strongest MPGs from 48

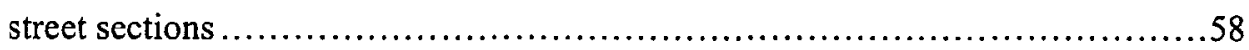

3.4 Medians of envelop fading ranges $(10 \%: 90 \%)$ for 48 street sections .............61

3.5 Medians of relative average powers for the three strongest MPGs from 147 street

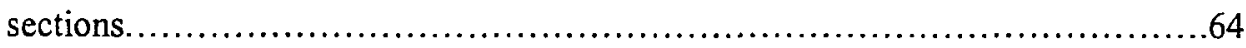

3.6 Medians of envelope fading range (10\%: $90 \%)$ for 147 street sections. ..........67

4.1 Percentage of street sections where the achievement of a BER of $10^{-3}$ would be possible with the different $\mathrm{Tx}$ locations in downtown Ottawa. 


\section{List of Symbols}

$\begin{array}{ll}a(t) & \text { Data spreading signal } \\ a_{q} & \text { Data spreading sequence } \\ \beta & \text { Self-interference component in the expression for bit error rate } \\ b(t) & \text { Data signal } \\ b_{q} & \text { Data sequence } \\ B_{c} & \text { Coherence bandwidth } \\ B_{D} & \text { Doppler bandwidth } \\ B_{s} & \text { Signal bandwidth } \\ c & \text { The speed of light } \\ C_{a}(\cdot) & \text { Discrete aperiodic autocorrelation function of the spreading sequence } \\ D_{1} & \text { Decision variable for a correlation receiver } \\ D_{\text {Rake }} & \text { Decision variable of a rake receiver } \\ D_{\text {Rake, norm }} & \text { Normalised Decision variable for a Rake receiver } \\ E(X) & \text { Expected value of random variable } X \\ E_{b} & \text { Energy per bit }\end{array}$

xiii 


\begin{tabular}{|c|c|}
\hline$f(\cdot)$ & $\begin{array}{l}\text { Sum of the continuous-time partial autocorrelation functions of the chip } \\
\text { waveform over a chip period }\end{array}$ \\
\hline$f_{D_{\max }}$ & Maximal Doppler frequency \\
\hline$f_{\text {sample }}$ & Frequency sample rate \\
\hline$\gamma_{k}$ & $\begin{array}{l}\text { Relative delay in chips of the } k^{\text {th }} \text { multipath group relative to the first, } \\
\text { rounded down to the nearest integer value }\end{array}$ \\
\hline$g(\cdot)$ & $\begin{array}{l}\text { Difference between the continuous-time partial autocorrelation functions } \\
\text { of the chip waveform over a chip period }\end{array}$ \\
\hline$h_{i}$ & Complex amplitude of multipath group having time -delay $\tau_{i}$ \\
\hline$h_{k, i}$ & Product of Envelope of $k^{\text {th }}$ multipath group and $i^{\text {th }}$ multipath group \\
\hline$h(t, \tau)$ & Channel impulse response \\
\hline$K$ & Number of received muptipath groups \\
\hline$L$ & Number of rake fingers \\
\hline$n(t)$ & Additive white Gaussian noise \\
\hline$N_{d}$ & Data spreading factor \\
\hline$N_{s}$ & Number of subintervals used in the semi-analytical technique \\
\hline$P\left(\tau_{k}\right)$ & Average Power of the multipath group at time-delay $\tau_{k}$ \\
\hline$P_{T_{\mathrm{c}}}$ & Rectangular pulse waveform with $T_{c}$ the chip period \\
\hline$P_{T_{d}}$ & Rectangular pulse waveform with $T_{d}$ the data symbol period \\
\hline$P_{e}$ & Probability of bit error \\
\hline$P_{s}^{L}$ & Lower bound on the probability of bit error \\
\hline
\end{tabular}

xiv 
$P_{E}^{U} \quad$ Upper bound on the probability of bit error

$Q(\cdot) \quad$ Gaussian-Q function

$r(t) \quad$ Equivalent low pass received signal

$R_{T_{c}}(\cdot), \widehat{R}_{T_{c}}(\cdot)$ Continuous-time partial autocorrelation functions of the chip waveforms

$R_{x x}(\tau) \quad$ Auto correlation of random variable $\mathrm{x}$

$R_{x y}(\tau) \quad$ Cross-correlation of random variable $\mathrm{x}$ and $\mathrm{y}$

$S(f) \quad$ Transmitted signal's spectrum

$\sigma_{I}^{2} \quad$ Variance of the self-interference

$\sigma_{\text {Rake }}^{2} \quad$ Variance of thermal noise in the rake receiver

$\sigma_{\tau} \quad$ RMS delay spread

$\tau_{i} \quad$ Excess time-delay of $i^{\text {th }}$ multipath group

$\tau_{k, i} \quad$ Difference between time-delays $\tau_{k}$ and $\tau_{i}$

$\bar{\tau} \quad$ Mean access delay

$\theta_{k, i} \quad$ Phase difference between multipath groups having time-delays $\tau_{k}$ and $\tau_{i}$

$t_{S R E} \quad$ Time interval between the consecutive sampled SRE

$T_{c} \quad$ Chip period

$T_{d} \quad$ Data symbol period

$\lambda \quad$ Wavelength

$u_{i}(c) \quad$ Upper bound on PDF of self-interference, conditioned on $C_{a}(1)=$ constant

$u_{i} \quad$ Unconditional upper bound on PDF of self-interference

$\mathrm{XV}$ 
$v_{i}(c) \quad$ Lower bound on PDF of self-interference, conditioned on $C_{a}(1)=$ constant

$v_{i} \quad$ Unconditional lower bound on PDF of self-interference

$v \quad$ Vehicle speed

$\omega_{c} \quad$ Angular carrier frequency

$W_{k, i} \quad$ Cross-correlation between interfering binary data sequences with time-

delays $\tau_{k}$ and $\tau_{i}$, respectively

xvi 


\section{List of Acronyms}

\begin{tabular}{ll} 
ACF & Autocorrelation function \\
AWGN & Additive white Gaussian noise \\
APDP & Average power delay profile \\
BER & Bit error rate \\
BPSK & Binary phase shift keying \\
CCF & Cross correlation function \\
CDF & Cumulative distribution function \\
ECDF & Estimated CDF \\
DSSS & Direct sequence spread spectrum \\
FCF & Frequency correlation function \\
FDD & Frequency Division Duplex \\
FR & Fading range \\
i.i.d & Independent and identical distribution \\
IRE & Impulse response estimate \\
LOS & Line-of-sight \\
MAI & Multiple access interference \\
MPCs & Multipath signal compoments \\
MPG & Multipath group \\
\hline
\end{tabular}

xvii 


$\begin{array}{ll}\text { MPSR } & \text { Multipath power sensitivity ratio } \\ \text { MRC } & \text { Maximal ratio combining } \\ \text { MS } & \text { Mobile Station } \\ \text { NLOS } & \text { Non-line-of-sight } \\ \text { pdf } & \text { Probability density function } \\ \text { PN } & \text { Pseudo-noise } \\ \text { RMS } & \text { Root mean square } \\ \text { SAT } & \text { Semi-analytical technique } \\ \text { SI } & \text { Self-interference } \\ \text { SISO } & \text { Single-input-single-output } \\ \text { SRE } & \text { Experimental system response estimate } \\ \text { TF } & \text { Transfer function } \\ \text { WSSUS } & \text { Wide-sense stationary uncorrelated scattering }\end{array}$

xviii 


\section{Chapter 1}

\section{Introduction}

Existing wireless cellular communication systems can be classified into three main types: First generation analogue systems, second generation (2G) digital voice systems, and third generation (3G) digital systems. Planning is now in progress for what is commonly referred to as "Beyond $3 \mathrm{G}(\mathrm{B} 3 \mathrm{G})$ Systems" or $4 \mathrm{G}$.

First generation analogue systems include the Advanced Mobile Phone Services system (AMPS) operating at $800 \mathrm{MHz}$ in North America, the Nordic Mobile Telephone (NMT) operating at $450 \mathrm{MHz}$ and $900 \mathrm{MHz}$ in Europe, and the Total Access Communication System (TACS) in Asia.

Second generation cellular systems are designed to use digital transmission for mostly voice traffic. They include the Pan-European Global System for Mobile communications (GSM) operating at $900 \mathrm{MHz}$, the Digital Communication Systems (DCS) operating at $1800 \mathrm{MHz}$, the IS-95 Code Division Multiple Access (CDMA One) system, operating at 800 and $1800 \mathrm{MHz}$ in North America, and the Personal Digital Cellular (PDC) system in Japan.

The 2G systems are mainly for voice communication. Data services offered by GSM systems are at low rates, being only $9.6 \mathrm{kbps}$, and $14.4 \mathrm{kbps}$ with advanced coding. In order to enable higher-rate data services, so-called $2.5 \mathrm{G}$ mobile systems are realized with packet switching instead of circuit switching for data transmission. The $2.5 \mathrm{G}$ mobile 
systems include the General Packet Radio Service (GPRS) and CDMA2000 1x RTT systems. GPRS is the world's most ubiquitous wireless data service. It is available now with almost every GSM network. Data rates offered by the GPRS can be as high as 171 kbps.

Third generation and beyond third generation cellular systems are designed to provide global mobility, and offer a wide range of services, including better quality voice, and high-speed data access to the Internet and multimedia services.

The International Telecommunication Union (ITU) initiated the process of standardization for $3 \mathrm{G}$ systems, and their standard is referred to as International Mobile Telecommunications 2000 (IMT-2000). In Europe, the European Telecommunications Standards Institute (ETSI) was responsible for another standard, the Universal Mobile Telecommunication System (UMTS) standard. Its radio access network is referred to as "UTRAN".

A wideband code division multiple access (WCDMA) scheme was selected for UTRAN air interface. CDMA utilizes spread spectrum (SS) modulation. The main characteristic of a spread spectrum system is that the transmitted signal has a bandwidth much larger than that of the baseband message signal. This allows bandwidth compression at the system receiver, with concomitant gains (processing gains) in signalto-noise ratio (SNR).

Depending on the method used to obtain a wideband signal, CDMA can be classified as being either of the direct sequence (DS) or frequency hopping (FH) type. When DS Spread Spectrum (DSSS) is employed, the transmit spectrum is spread through multiplying a narrowband message signal with a pseudo-noise (PN) sequence, resulting 
in a wideband signal. In FHSS, a PN sequence defines the instantaneous transmission frequency. The transmit frequency therefore changes pseudo-randomly to avoid interference or to reduce collision likelihood.

UMTS WCDMA is a DSSS system with Frequency Division Duplex (FDD) operating in paired frequency bands for uplink and downlink communication.

As a result of increasing demand for greater mobile system capacities, and higher data rate services, IMT2000 systems and yet-to-be defined B3G (sometimes referred to as IMTAdvanced) mobile systems need to be expanded into frequency bands other than the current bands near $2 \mathrm{GHz}$. Such expansion would be a pre-cursor to the next step in the evolution of mobile communications systems, and the definition of $4^{\text {th }}$ generation digital systems. As a result, there is a need for the estimation of expected mobile link performance at frequencies other than in the $2 \mathrm{GHz}$ band. Researchers at Industry Canada's Communications Research Centre (CRC) have been conducting radio propagation research relevant to such expansion for a number of years. They have conducted both Continuous Wave $(\mathrm{CW})$ and wideband radio channel sounding measurements at $1.9 \mathrm{GHz}$ and $5.8 \mathrm{GHz}$, for comparison purposes in downtown Ottawa prior to the initiation of work for this thesis project. Their results are to be published in early $2007[2]$.

Whereas the work reported in [2] involved measurements in the two frequency bands on different days, the quasi-simultaneous measurement of propagation data in the $2 \mathrm{GHz}$ and $6 \mathrm{GHz}$ bands is the subject of work reported in this thesis. The time-varying characteristics of urban vehicular-mobile channels in both bands are analysed and reported. 
In addition, both well-known methods and new techniques developed at CRC [3] are used for the prediction of DSSS performance on the measured channels. For assessing the requirements of un-embellished, lower data rate IMT2000 systems, if operated in higher frequency bands, bit-error-rate (BER) performance predictions are made for transmissions with low symbol rate and high spreading factors. For such cases, selfinterference (SI) that results from multipath propagation is insignificant, and need not be considered. Performance estimation procedures are well known [1], and results could be of interest, when we simply want to expand existing systems into new bands. For the transmission of higher data rates in IMT2000 bandwidths, however, the number of chips per data symbol (i.e., the spreading factor) must be decreased. As a result, the crosscorrelation of shorter segments of the system spreading sequences with the local reference during receiver processing produces sidelobes with high power levels. The combination of shorter symbol durations for higher data rates and higher-powered cross correlation sidelobes leads to SI at levels that can no-longer be neglected in BER predictions. To account for SI, the semi-analytical technique (SAT) developed in [3] is used in the work of this thesis to estimate and compare higher-data rate DSSS mobile link BER performance expected on mobile links with IMT2000 bandwidths in the 2 and 6 $\mathrm{GHz}$ frequency bands. When the SAT is employed, SI can accurately be accounted for. Additionally, channel models, which are sometimes simplistic and inaccurate, can be replaced by the available quasi-simultaneous 2 and $6 \mathrm{GHz}$ band time series of measured propagation data. Results from this work apply to the study of an operating mode that could be used in IMTAdvanced systems, wherein time-division multiple access is 
employed to allow high data rate transmission within short time intervals (e.g. for downloading video information) to single users.

\subsection{Objectives}

As explained in the foregoing, the objectives of the work reported in this thesis include:

- The conduct of quasi-simultaneous propagation measurements in the $2 \mathrm{GHz}$ and 6 $\mathrm{GHz}$ frequency bands in an urban area (downtown Ottawa);

- Analysis of the measured data for the estimation and comparison of radio propagation characteristics in the 2 and $6 \mathrm{GHz}$ bands;

- The prediction of BER performance on DSSS mobile links operating with both low $\left(N_{d}=7\right)$ and high $\left(N_{d}=128\right)$ spreading factors, for applications as discussed in the foregoing section; and

- The interpretation and explanation of predicted BER performance characteristics, using knowledge gained from the analysis of the measured propagation data.

\subsection{Contributions}

- Calibration of a newly-developed quasi-simultaneous 2 and $6 \mathrm{GHz}$ band pseudo noise radio channel sounding system;

- Statistical analysis of measured radio propagation data to derive estimates of parameters of the transmission channel (i.e. the Transmitter (Tx)-radio channelReceiver ( $\mathrm{Rx}$ ) combination), such as impulse response functions, envelope fading statistics, and RMS delay spreads; 
- Prediction and comparison of BER performance for Rake reception on DSSS mobile links operating in the 2 and $6 \mathrm{GHz}$ bands with both low and high spreading factors (i.e. high date rates and low data rates) in IMT2000 bandwidths.

- An assessment of the requirement for enhanced channel protection for $3.84 \mathrm{MHz}$ IMT2000 systems operating in higher frequency bands, and for such requirements arising from the use of low spreading factors to allow higher data rate transmissions in both frequency bands; and

- Conclusions regarding the relationship between specified radio channel characteristics and performance on mobile DSSS radio links.

\subsection{Organisation of this thesis}

Background information regarding radio channel characterisation, classification, and the operation of Rake receivers on DSSS mobile radio links is introduced in Chapter 2. The quasi-simultaneous measurement system, calibration tests, and the results from statistical analysis of measured data are presented in Chapter 3. The SAT technique for BER prediction in cases where SI is significant and its application are reviewed in Chapter 4, and the predictions of BER performance on the measured mobile radio channels for both low data rate (Original IMT2000) and higher data rate (IMTAdvanced) are reported. A summary, conclusions, and recommendations for further research are presented in Chapter 5. 


\section{Chapter 2}

\section{Background}

Basic information about radio channel characteristics, multipath interference and Rake receivers is presented in this chapter. A literature review pertinent to channel measurements and Rake receiver performance is also included.

\subsection{Channel characteristics}

Knowledge of radio channel characteristics is essential in the design and planning of digital cellular systems. In wireless telecommunications, multipath is the propagation phenomenon that results in the reception of a radio signal over two or more propagation paths. When a radio signal travels between a transmitter $(\mathrm{Tx})$ and a receiver $(\mathrm{Rx})$, it suffers reflection, diffraction and scattering by objects in the environment. Fig.2.1 shows a diagram of a downlink radio channel in a mobile system. Reflection occurs when a signal encounters a surface that is large relative to the wavelength of the signal; diffraction occurs at the edge of an impenetrable body that is large compared to the wavelength of the radio wave; and scattering occurs when the signal impinges upon an object whose size is on the order of the wavelength of the signal or smaller. Multipath propagation results in the received signal being composed of time-delayed replicas of the transmitted signal. 


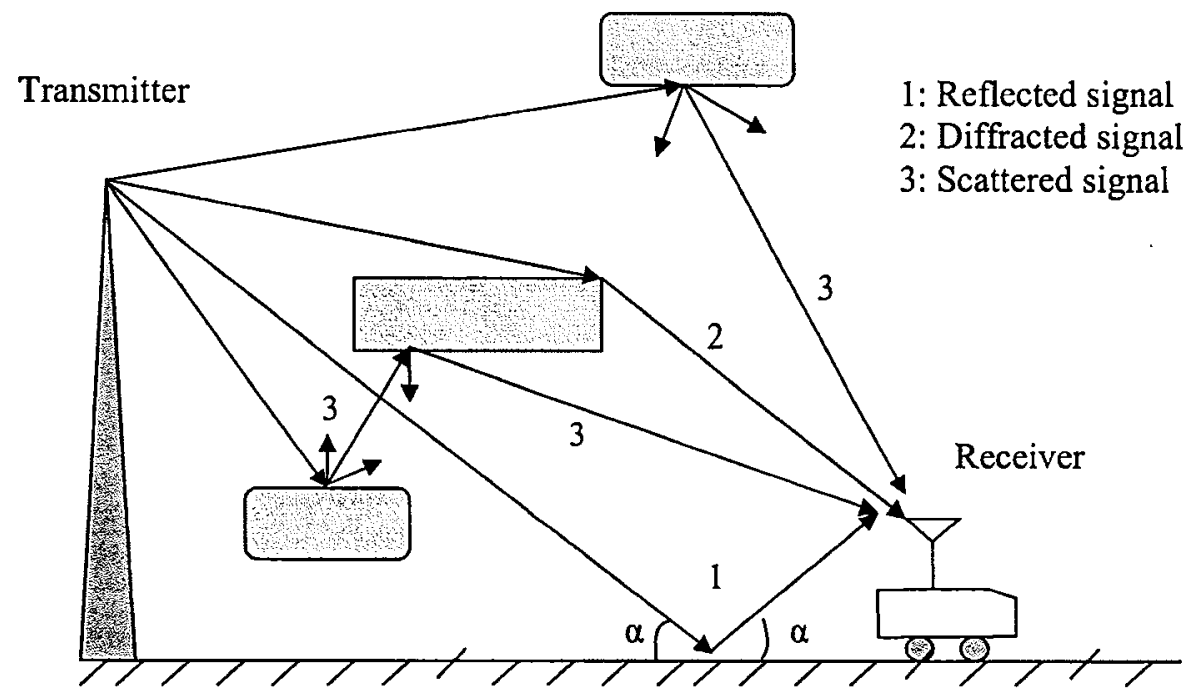

Fig.2.1: A diagram of a downlink radio channel in a mobile system

A radio link can be characterized by the following parameters [4] [5]:

- Path loss: resulting from spreading of electromagnetic (EM) energy and from the EM energy loss due to penetration of objects;

- Large-scale fading (or shadow fading): the spatially varying attenuation of received signal power over regions in space on the order of 10 wavelengths or larger;

- Small-scale fading: the spatially varying attenuation of received signal power over regions in space that is smaller than about 10 wavelengths.

Generally, a radio channel can be modelled as a linear time-varying (LTV) filter that can be characterised by its time-varying impulse response $h(t, \tau)$, where $t$ denotes time, and $\tau$ denotes propagation delay. As a result of the movement of a mobile station (MS) and other interacting objects, the characteristics of mobile radio channels must be represented by statistical channel models, such as the model commonly referred to as the Gaussian wide sense stationary uncorrelated scattering (GWSSUS) model. This model 
assumes that the channel process is wide-sense stationary (WSS), meaning that the time autocorrelation of the channel's impulse response is independent of time, and depends only on delay. It also assumes uncorrelated scattering (US), meaning that the correlation of random variations across the channel bandwidth is invariant over frequency, being dependent only on frequency spacing. The average power delay profile (APDP) of a time-varying channel, which is the expected value of the received power at a certain delay, is one parameter that is frequently used to characterize mobile radio channels. Several other multipath channel parameters can be estimated from the APDP. That information can be used to predict error performance characteristics of digital transmission on the associated mobile radio channel.

Coherence bandwidth and Root Mean Square (RMS) delay spread are parameters that describe the time dispersive nature of the channel in a local area. Time dispersion is caused by multipath propagation delays. Its properties can be described by the mean excess delay $\bar{\tau}$ and the RMS delay spread $\sigma_{\tau}$. The mean excess delay is the first moment of the APDP and can be written as $\bar{\tau}=\frac{\sum_{k} P\left(\tau_{k}\right) \tau_{k}}{\sum_{k} P\left(\tau_{k}\right)}$, where $P\left(\tau_{k}\right)$ is the average power at delay $\tau_{k}$. The RMS delay spread is the square root of the second central moment of the APDP and can be written as $\sigma_{\tau}=\sqrt{\overline{\tau^{2}}-(\bar{\tau})^{2}}$, where $\overline{\tau^{2}}=\frac{\sum_{k} P\left(\tau_{k}\right) \tau_{k}^{2}}{\sum_{k} P\left(\tau_{k}\right)}$

The coherence bandwidth $B_{c}$ represents the maximum frequency separation for which the frequency domain channel responses at two frequencies remain strongly correlated during fading in time. The coherence bandwidth has a reciprocal relation with 
the RMS delay spread if the channel exhibits truely GWSSUS fading characteristics [5], in which the Probability Density Function (PDF) of the channel complex envelop has zero mean, so that narrowband envelope fading follows a Rayleigh distribution.

When the transmitted signal bandwidth is very much smaller than the channel coherence bandwidth, i.e. the symbol period of the transmitted signal is much bigger than the delay spread, the signal suffers flat fading when it is transmitted over the channel; otherwise, if the transmitted signal bandwidth is comparable to or larger than the channel coherence bandwidth, i.e. the symbol period of the transmitted signal is comparable to or smaller than the delay spread, the signal suffers frequency selective fading when it is transmitted over the channel. Fig.2.2 shows the above two situations, where $F C F$ denotes the Frequency Correlation Function, $B_{c}$ is the channel coherence bandwidth; $S(f)$ is the transmitted signal's spectrum, $B_{s}$ is the transmitted signal bandwidth

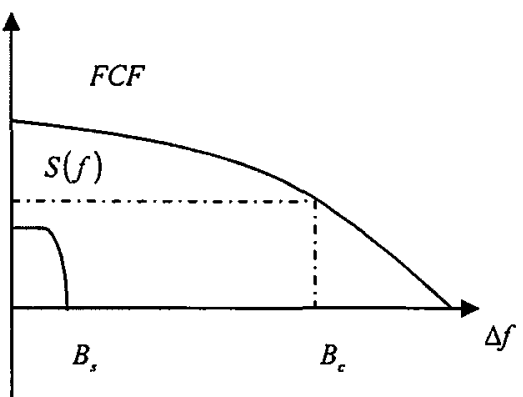

(a)

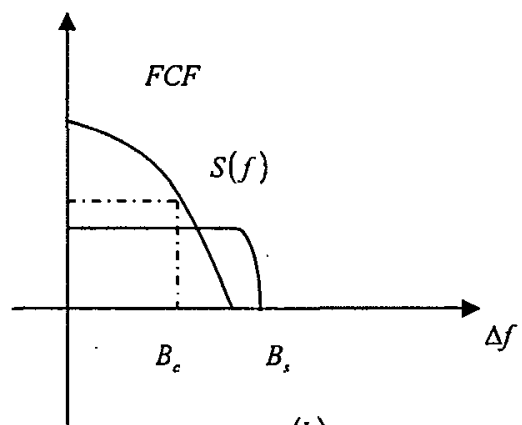

(b)

Fig.2.2: (a) transmitted signal with flat fading and (b) transmitted signal with frequency selective fading.

Due to the movement of a MS and interacting objects, the received frequency at the mobile is different from the transmitted frequency because of the Doppler phenomenon. Since the received multipath components arrive along many different paths, the relative 
velocity of the MS with respect to various interacting objects causes the different components to have different Doppler shifts. This received signal is therefore spread in frequency and exhibits what is referred to as a Doppler Spread, having a $3 \mathrm{~dB}$ bandwidth denoted $B_{D}$. Doppler spread and coherence time are parameters that describe the time varying nature of the channel. Coherence time represents the maximum time separation for which the channel impulse responses at two time instants remain strongly correlated. The Doppler spread and coherence time are inversely proportional to each other on a GWSSUS channel. When the symbol period of the transmitted signal is equal or bigger than the channel coherence time, i.e. the transmitted signal bandwidth is smaller than the channel Doppler spread, the signal suffers fast fading, or time selective fading when it is transmitted over the channel; otherwise, if the symbol period of the transmitted signal is much smaller than the channel coherence time, i.e. the transmitted signal bandwidth is much bigger than the channel Doppler spread, the signal suffers slow fading when it goes through the channel. The implication of slow fading is that the phase of the received signal can be considered approximately constant over the symbol duration.

Irreducible error rate floors are exhibited on both time-selective, and frequencyselective fading channels, as well as on channels that exhibit both types of selective fading, referred to as doubly-selective fading channels. Such error rate floors maintain BERs at a constant level, no matter how large the average signal to noise ratio at the $\mathrm{Rx}$ becomes. If such floors are at an unacceptable level, channel protection, such as equalization, coding, or interleaving is required to achieve better BER performance. 


\subsection{Interference in DSSS systems}

Error performance in DSSS systems with multiple users is interference-limited due to multiple access interference (MAI) generated by different users transmitting within the same bandwidth simultaneously.

Wideband signals from different users are separated by means of spreading sequences, also known as spreading codes, which are unique to each user. Each user is assigned an individual spreading code at the time of call initiation. This spreading code is used both for spreading the narrowband message signal at the time of transmission and dispreading the received signal.

Spreading codes are usually orthogonal, but there are some scenarios under which the orthogonality cannot be controlled. For example, on a downlink, the BTS transmits signals to all users synchronously and this preserves orthogonality. The orthogonality, however, is not preserved between different components arriving from different multipath sources. Also on the uplink, when different users transmit asynchronously, the orthogonality of the spreading codes is not preserved.

Hence, the asynchronous transmission or the time-varying nature of the mobile radio channel may partially destroy orthogonality. The non-orthogonal nature of the codes results in MAI, which degrades the performance of the system.

Frequency selective fading mobile radio channels also give rise to SI due to time spreading of the desired user's signal in DSSS systems. SI occurs when data symbol durations are less than the maximum multipath delay on the channel.

Third generation wireless systems transmitting at high bit rates, such as $2 \mathrm{Mbps}$, experience significant channel-induced SI. In DSSS systems, with an increase in data rate 
for a fixed chip rate, there is a decrease of the spreading factor, i.e. a decrease of the number of chips per symbol interval. This in turn results in increased SI because the cross-correlation of shorter partial sequences results in higher sidelobe levels.

Although increasing transmitted power to the desired user can mitigate MAI, reductions in SI cannot be achieved by this method, since the power of both the direct and multipath signals is simultaneously increased. Work reported in this thesis focuses on assessing and comparing technology requirements for the achievement of acceptable error performance on single mobile radio links in the currently used $2 \mathrm{GHz}$ frequency band and a higher frequency band $(6 \mathrm{GHz})$, considered as the highest band feasible for possible allocation to mobile services in the future. MAI is not considered since interest is only in the physical channel characteristics, rather than effects that result from how the channel is used.

\subsection{Rake receivers in a DSSS system}

Rake receivers take advantage of multipath dispersion to yield diversity gains. As described above, on a multipath channel the received signal is composed of time-delayed replicas of the transmitted signal, which are attenuated in amplitude and shifted in phase. All multipath signal components (MPCs) within the time resolution' of Rx will be seen as a single multipath group (MPG). That is, the energy within an MPG is the combination of the energy of all of the undistinguishable MPCs. In order to combine the different MPGs, a Rake receiver needs the knowledge of channel parameters such as, number of MPGs,

\footnotetext{
${ }^{1}$ Reciprocal of the RF bandwidth of transmitted signal
} 
their delays and their (complex-valued) amplitudes. Fig.2.3 shows a block diagram of a Rake receiver.

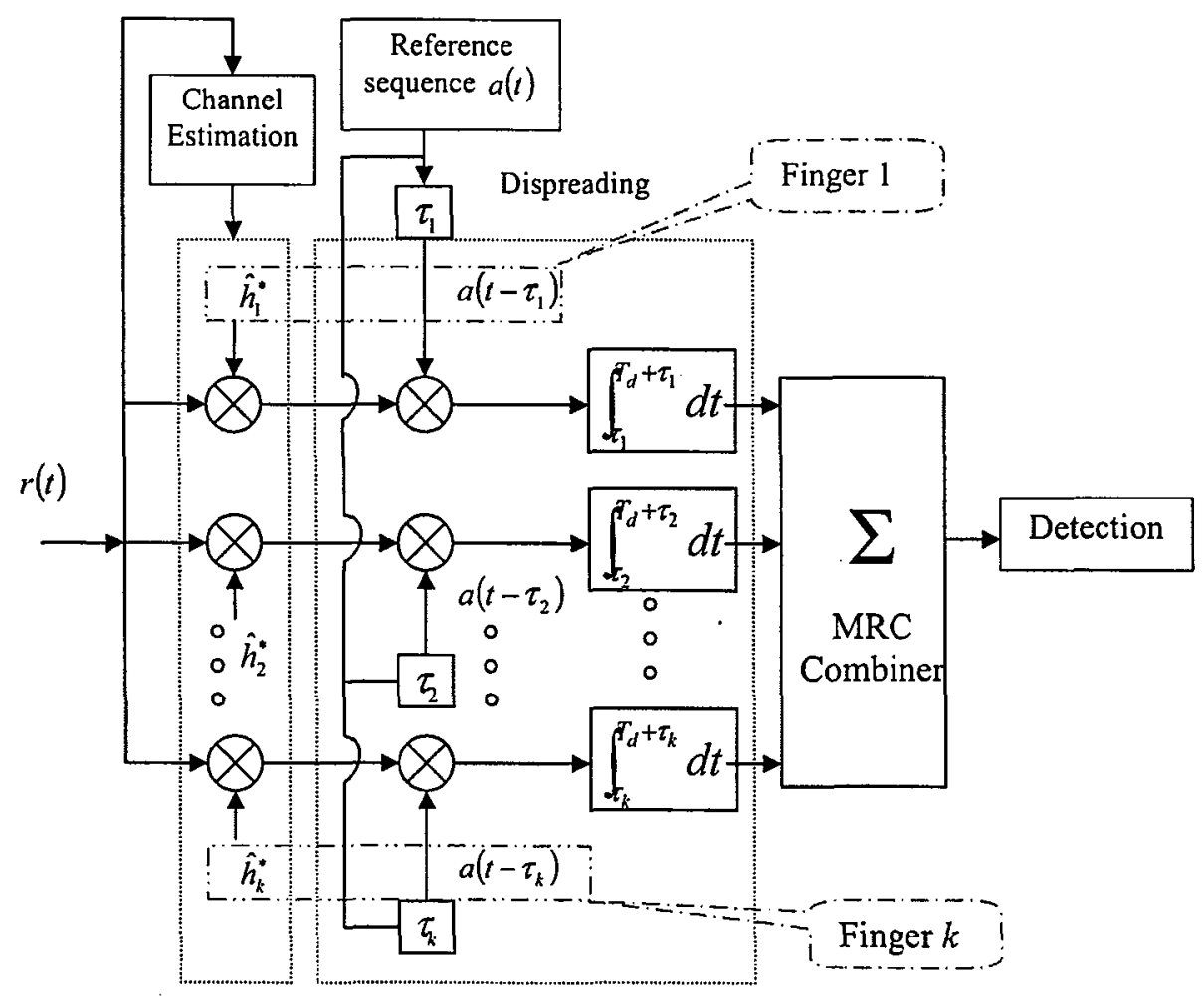

Fig.2.3: Block diagram of the Rake section in a typical DSSS receiver.

Channel estimation is an important consideration in BER analyses for a Rake receiver. However, there are numerous techniques described in previous papers that can be used to accurately track and select the time-delays of received MPGs intended for Rake combining [7] [8] [9]. In this thesis, it is therefore assumed that the Rake receiver is able to accurately determine the time-delays of received MPGs. 
The Rake receiver in Fig.2.3 shows $k$ Fingers; one Rake Finger is assigned to each of the MPGs. With maximum ratio combining (MRC) technology, all selected MPGs are combined to form a composite signal that is expected to have substantially better a signalto-noise ratio.

Considering a single-input-single-output system [3], the transmitted signal for a single user with BPSK modulation can be written as $s(t) \cos \omega_{c} t$, where $s(t)=\sqrt{\frac{2 E_{b}}{T_{d}}} b(t) a(t)$, $b(t)$ is the transmitted data signal and can be written as $b(t)=\sum_{q=-\infty}^{\infty} b_{q} p_{T_{d}}\left(t-q T_{d}\right)$, and $a(t)$ is the spreading signal and can be written as $a(t)=\sum_{q=-\infty}^{\infty} a_{q} p_{t_{s}}\left(t-q T_{c}\right),\left\{b_{q}\right\},\left\{a_{q}\right\}$ are sequences of elements from the set $\{-1,1\}, p_{T_{d}} p_{T_{c}}$ are rectangular pulses with $T_{d}$ the data symbol period and $T_{c}$ the chip period, respectively. The spreading factor is $N_{d}=r_{d} / T_{c}$ the number of chips in one symbol interval, $\omega_{c}$ is the carrier frequency and $E_{b}$ is the energy per bit. Note that in this representation it is assumed that $\int_{d}^{T_{d}} a^{2}(t) d t=T_{d}$.

Since the signal received over a wireless channel is composed of time-delayed replicas of the transmitted signal, the equivalent low pass received signal with $K$ MPGs can be written as:

$$
r(t)=\sum_{k=1}^{K}\left|h_{k}\right| e^{j \varphi_{k}} s\left(t-\tau_{k}\right)+n(t)
$$

where $\tau_{k}=\tau_{k}(t)$ and $\left|h_{k}\right|=\left|h_{k}(t)\right|$ are the time-delay and amplitude of the $\mathrm{k}^{\text {th }}$ MPG. The argument " $t$ " of $h_{k}(t)$ and $\tau_{k}(t)$ has been dropped under the assumption of slow 
fading. $\varphi_{k}$ is the phase shift that results from interactions with objects along the kth propagation path. The term $n(t)$ represents low pass additive white Gaussian noise (AWGN) with zero mean and power spectral density of $N_{o}$.

With the assumption of error-free channel estimation, the $k^{\text {th }}$ estimated MPG, $\hat{h}_{k}$, equals the $k^{\text {th }}$ MPG, $h_{k}$. Therefore, for the first transmitted data symbol, the decision variable at the output from a Rake combiner having L Fingers can be written as:

$$
D_{\text {Rake }}=\sum_{i=1}^{L} \int_{\tau_{i}}^{\tau_{d}+\tau_{i}} \operatorname{real}\left\{r(t) h_{i}^{*} a\left(t-\tau_{i}\right)\right\} d t
$$

After substitution of (2.1), one can write

$$
\begin{aligned}
& D_{\text {Rake }}=\sqrt{2 E_{b} T_{d}} b \sum_{i=1}^{L}\left|h_{i}\right|^{2}+\sqrt{2 E_{b} T_{d}} \sum_{i=1}^{L} \sum_{k=1, k \neq i}^{K} h_{k, i} \frac{1}{T_{d}} \cos \theta_{k, i} W_{k, i}+\eta_{\text {Rake }} \\
& \text { and when normalized by } T_{c} \sqrt{\frac{2 E_{b}}{T_{d}}} \text {, equation (2.2) becomes } \\
& D_{\text {Rakc ,uorm }}=N_{d} b \sum_{i=1}^{L}\left|h_{i}\right|^{2}+\sum_{i=1}^{L} \sum_{k=1, k \neq i}^{K} h_{k, i} \frac{\cos \theta_{k, i}}{T_{c}} W_{k, i}+\frac{1}{T_{c}} \sqrt{\frac{T_{d}}{2 E_{b}}} \eta_{\text {Rake }}
\end{aligned}
$$

where $h_{k, i}=\left|h_{k} \| h_{i}\right|$.

The first term is the combined signal energy from all L Rake Fingers; the second term represents the self-interference caused by multipath propagation, where the phase term $\theta_{k, i}=\varphi_{k}-\varphi_{i}$ is assumed to be uniformly distributed on $[0,2 \pi]$, and $W_{k, i}=\int_{d_{i}}^{\pi_{d}+\tau_{i}} b\left(t-\tau_{k}\right) a\left(t-\tau_{k}\right) a\left(t-\tau_{i}\right) d t$ is the cross-correlation between interfering binary sequence $k$ and the $i^{\text {th }}$ reference sequence; the third term is the result of the low pass AWGN noise and 
$\eta_{\text {Rake }}=\sum_{i=1}^{L} \int_{\tau_{i}}^{T_{d}+\tau_{i}}$ real $\left(n(t)\left|h_{i}\right| e^{-j \varphi_{i}} a\left(t-\tau_{i}\right)\right) d t$

Since $n(t)=n_{r}(t)+j n_{i}(t), E\left(n_{r}(t) n_{r}(\tau)\right)=N_{0} \delta(t-\tau), E\left(n_{i}(t) n_{i}(\tau)\right)=N_{0} \delta(t-\tau)$

and $E\left\{n_{r}(t) n_{i}(t)\right\}=0$, one can derive that the variance of $\eta_{\text {Rake }}$ is:

$$
\sigma_{\text {Rake }}^{2}=\sum_{i=1}^{L}\left|h_{i}\right|^{2} N_{0} T_{d}
$$

and the normalized noise variance is

$\sigma_{\text {Rake }, \text { norm }}^{2}=\left.\frac{N_{0} N_{d}^{2}}{2 E_{b}} \sum_{i=1}^{L} h_{i}\right|^{2}$

Under the assumption that the relative time-delay between received MPGs is an integer multiple of the chip period $T_{c}$, the sequence cross-correlation can be modelled as having a binominal PDF. Fig.2.4 shows the comparison between binominal PDF and the PDF of results from the cross-correlation of two sequences of length 5 shifted by an integral number of chips with respect to each other. 


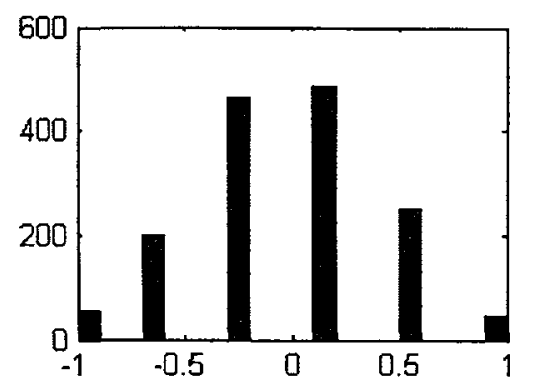

(a)

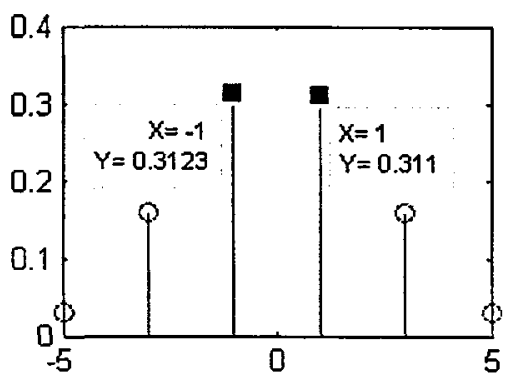

(c)

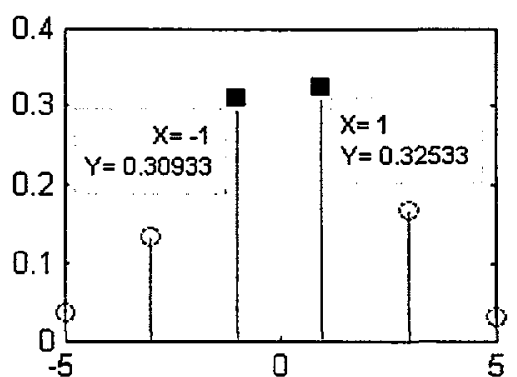

(b)

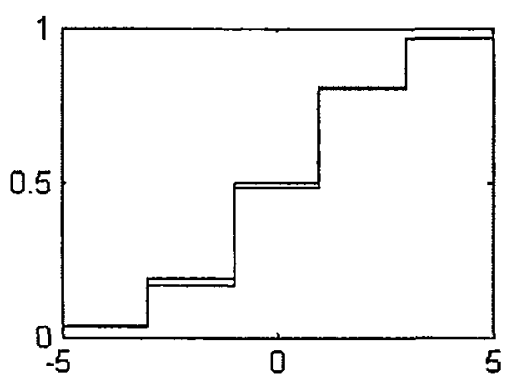

(d)

Fig.2.4: Comparison between a binominal distribution and the distribution of crosscorrelation of two integer-shifted 5-chip long sequences.

(a): Histogram of cross-correlation between two integer-shifted 5-chip sequences;

(b): PDF of cross-correlation between two integer-shifted sequences;

(c): PDF of a binomial distribution with length 5;

(d): Comparison between binominal CDF and the CDF of cross-correlation of two integers- shifted 5-chip sequences.

As the length of cross-correlated binary sequences increases the PDF of the crosscorrelation approaches that of a Gaussian distribution more and more closely [3]. 


\subsection{Literature Review}

There are two subprojects involved in the work of this thesis. One is about measurement of channel characteristics for urban microcells. The other is about prediction of DSSS link performance using the measured data.

Literature review shows that channel measurements have been studied for a long time and the measurement-based characterization of mobile channels has been reported frequently. In 1972, Cox reported a pseudo random binary sequence (PRBS) measuring system to measure multipath delay and Doppler characteristics at $910 \mathrm{MHz}$ in suburban mobile radio environments [10]. Small-scale statistics of multipath for vehicle travel distances in the order of $30 \mathrm{~m}$ along streets were characterised by Average Power Delay Profiles (APDPs) made up of over 200 individual channel Impulse Response Estimated (IREs). Cumulative distributions of the variation of signal amplitudes at fixed delays and radio frequency Doppler spectra at fixed delays. Cox also reported similar studies for heavily built-up urban mobile radio environments in [11] [12], and suggested that, for an $\mathrm{RF}$ bandwidth of $10 \mathrm{MHz}$, it appeared reasonable to model urban mobile radio channels using a GWSSUS model for intervals along the street of up to $30 \mathrm{~m}$.

Bultitude and Bedal in 1989 first reported propagation characteristics on urban microcellular-type channels at $910 \mathrm{MHz}$ [13]. The paper showed that multipath propagation conditions were significantly less severe compared with conventional urban mobile radio channel and the RMS delays were reduced by a factor of four. In addition, the paper reported that microcellular-type channels had Rician, rather than Rayleigh, envelope fading characteristics, and correspondingly different frequency correlation statistics. 
Gurgin and Rappaport reported propagation channel measurements at higher frequency band $5.85 \mathrm{GHz}$ around residential areas [14]. Results of path loss and building penetration loss were presented, specific effects of foliage shadowing, house shadowing etc were studied. Also, Blackard, Rappaport and Seidel et.al in [15] presented results of wideband path loss and delay spread measurements for two representative microcellular environments at $1.9 \mathrm{GHz}$. Measured RMS delay spreads were found to be a strong function of the antenna height. The paper indicated a trade off between coverage area and delay spread considerations. Kalliola and Vainikainen in [16] reported directional channel measurements at $2.15 \mathrm{GHz}$ with a spherical array and a wideband channel sounder. A wideband signal was transmitted using a single antenna, and received separately with each of the 32 elements of the spherical array. The paper showed variation of the local azimuth and elevation power distribution when the mobile moved in the urban environment.

None of the above results give the comparison of urban channel characteristics at different frequencies. A paper by Bultitude, and Schenk et. al in [2], however considered the measurement - based comparison of urban microcellular mobile radio channel characteristics at $1.9 \mathrm{GHz}$ and a higher frequency, $5.8 \mathrm{GHz}$, Continuous Wave (CW) measurements and wideband sounding measurements at both frequencies were conducted. Characteristics that were reported include transmission loss, RMS delay spread, frequency correlation and envelope fading statistics. The wideband measurements were conducted in downtown Ottawa, and were from street sections within two city blocks of the Tx site. At both frequencies, transmission with an EIRP of $+36 \mathrm{dBm}$ was from a biconical antenna mounted at $7.8 \mathrm{~m}$ above the sidewalk along urban street. Reception at 
each centre frequency was via a tuned quarter-wavelength monopole mounted in the centre of a minivan roof. Measurements at $1.9 \mathrm{GHz}$ and $5.8 \mathrm{GHz}$ were made on different days. The measured data at both frequencies were recorded from approximately the same street sections. From the Tx, 511 chip PN sequences were transmitted at a rate of 5 Mchips/s. Off -air signals at the Rx were quadrature down-converted and sampled at 10 Msamples/s. Time series data were recorded at 450 impulse response estimates per second. Conclusions were that RMS delay spreads were approximately the same in the two frequency bands, but that transmission loss would be approximately $10 \mathrm{~dB}$ greater at $6 \mathrm{GHz}$.

In order to improve on the latter work, in which measurements were conducted in two bands on different days, by obtaining measurements of channel characteristics under almost exactly the same conditions in 2 and $6 \mathrm{GHz}$ frequency bands, quasi-simultaneous measurements are conducted in the work of this thesis.

Prediction of Rake receiver performance in DSSS systems has also been studied for many years. In order to accurately account for SI, Adnani in [3] presented a new computationally efficient semi-analytical technique (SAT) for the prediction of Rake receiver performance on fading multipath channel. This technique avoids some commonly used assumptions. For example, often relative time-delays between any two received MPGs are assumed to be an integer multiple of the chip period, received MPGs are assumed to have equal average powers and the fading of MPGs is assumed to be identical, and independently distributed (i.i.d) and Rayleigh. These assumptions have been made to allow the development of channel models that are simplified with respect to 
reality in order to achieve simplicity and mathematical tractability, but [3] shows that they can lead to significant errors in link performance prediction.

There are numerous reports and publications based on those assumptions. For example, Boujemaa and Siala [17] obtained a simplified expression for Rake receiver performance with the assumption of chip-spaced MPGs. Chip-spaced MPGs can result in SI that can be characterised by a binominal distribution, and make analysis of error performance much more easy. On real mobile radio channels, multipath fading makes the delay of received MPGs vary randomly. It is obvious therefore the assumption of received chip-spaced MPGs is not true. Also Torrieri in [18] analysed the performance of DSSS systems with long pseudo noise sequences. The SI was modelled as a Gaussian distribution for spreading factors greater than 64 . The paper presented an approach that assumed that if the spreading factor increases to a greater enough value, the binominal distribution of SI can be modelled as a Gaussian distribution. In [19], Cheun derived error probability expressions for BPSK and QPSK DSSS systems employing random spreading sequences with Rake receiver. The fading of received MPGs was modelled as Rayleigh and i.i.d. The analysis of measured data shows that the assumption of equal power and i.i.d Rayleigh fading for received MPGs is seldom valid. Again, the paper modelled SI as a Gaussian distribution. To avoid the Gaussian approximation, Hwang and Lee [20] analysed the performance of Rake receiver in DSSS systems operating at high data rates with low processing gain (i.e. low spreading factors) by using a simulation-based technique that averaged performance over random spreading codes. Guenach and Vandendorps [21] analysed the downlink BER performance of a BPSK-based WCDMA 
with Rake receiver. Time-delayed MPGs were assumed as Rayleigh fading. That is not always true also. Simulation-based BER performance results were presented in that paper.

Lehnert and Pursley in [6] created a semi-analytical technique to estimate the effects of MAI on performance in a DSSS system for random spreading sequences when a correlation receiver was used on a non-fading channel. An expression for the decision statistic of a cross-correlation Rake receiver was presented. This enabled the conditional density function of MAI to be obtained. The interference due to $2^{N_{d}}$ possible sequences of length $N_{d}$ was divided into $N_{s}$ classed to determine arbitrarily tight upper and lower bounds distribution of MAI leading to tractable expressions for BER. Through extending the technique in [6] for MAI in non-fading environment, Adnani in [3] developed a semianalytical technique to make BER predictions taking the effects of SI into accurate account. A comprehensive analysis of SI for one single transmitter with random spreading sequences in DSSS systems was reported. The expression of the decision statistic for a Rake receiver and the conditional probability density function of SI were presented, and the expressions for BER bounds were also reported. Simulation-based verification of the SAT included a Monte-Carlo simulation to estimate the BER for the case in which only one of two equal power MPGs was processed in a correlation receiver. The verification results showed that the SAT provided reliable bounds on BER. It was found that as the number of subintervals $N_{s}$ that are used to represent the probability density functions for SI, increases the upper and lower bounds on the PDF for the SI converge allowing a single BER result. Simulation results showed that the upper and lower bounds of BER estimated using the SAT with $N_{s}=50$ were very close to each other. BER performance results obtained using the SAT were compared with those 
obtained under different assumptions in the previous literature in [3]. The comparisons showed that the assumption of chip-spaced MPGs and SI Gaussian assumptions both led to the overestimate of BER performance. The BER performance evaluated by assuming equal powered MPGs gave the maximum diversity gain where the worst result was obtained when only one of the equal powered MPGs was processed. The reason was reported to be that the SI was at its maximum value when MPGs had equal average power.

Through the review of available literature on mobile propagation channel measurements, channel modelling and prediction of DSSS mobile link performance, it is clear that most available channel models do not accurately represent the characteristics that prevail on urban microcellular channels. Thus, it is also clear that any performance predictions based on them to meet the stated objectives of the project would not be accurate.

Therefore, time series measurements are needed to avoid the requirement for the use of simplified channel modelling. Estimation of channel impulse response and other time delay parameters, such as APDP, MPGs and RMS delay spread provides necessary information for the design of mobile communication systems.

Prediction of BER performance in the work of this thesis includes two parts: one part is $\mathrm{BER}$ prediction at 2 and $6 \mathrm{GHz}$ with low spreading factor of 7 (i.e. high data rates), the methods outlined in [3] are adopted in order to accurately account for SI in IMTAdvanced systems. Interpretation and comparison of BER results at two frequencies are done. Assessment of the requirement for enhanced channel protection arising from the use of low spreading factors to allow higher data rate transmissions is also done in the 
thesis. In addition, BER prediction that applies to the simple movement of IMT2000 systems with high spreading factor of 128 (i.e. low data rates) to higher frequency bands is done in the work of this thesis. Without consideration of SI, it doesn't require any special treatment. Comparisons and assessment of error performance in frequency bands near 2 and $6 \mathrm{GHz}$ are presented. 


\section{Chapter 3}

\section{Experimental Set-up and Data Analysis}

The channel measurement system used for the work reported in this thesis is described in this chapter. Then, the method for using the measured data to estimate radio channel characteristics is presented, and analysis results are shown.

The propagation parameters of interest in radio channel characterization for the estimation of Rake receiver performance include the channel impulse response, Rake finger envelope fading time series and RMS delay spreads. Following a description of the measurement system in Section 3.1, the data analysis procedures that were used to estimate these characteristics and data analysis results, which quantitatively report the parameters of interest, are reported in Section 3.2.

\subsection{The Measurement Equipment}

This section includes a description of the channel measurement system, calibration tests and the field measurement setup. 


\subsubsection{Description of the channel measurement system}

Mobile radio propagation channels can be modelled as linear time varying filters. The channel impulse response changes in time and delay, and can be denoted as $h(t, \tau)$, as shown in Fig.3.1.1.

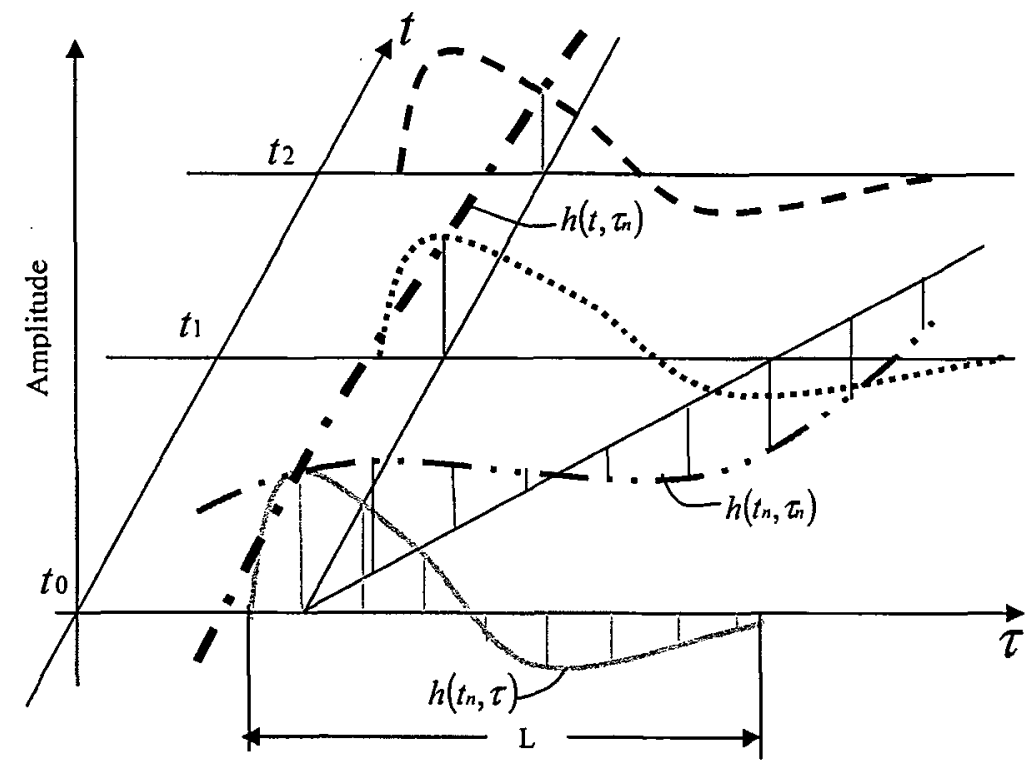

Fig.3.1.1: Impulse response for a linear time varying filter.

The figure shows impulse responses at instant time $t_{0}, t_{1}$ and $t_{2}$, reflecting the channel behaviour at different times. The curve with "Dash Dot" shows the channel behaviour as a "time series" at fixed delay $\tau_{n}$, and the curve with "Dash Dot Dot" shows the channel behaviour of impulse response following time and delay series.

The impulse response of a linear filter can be obtained using a cross correlation technique when the input signal $x(t)$ is a white noise [22].

$R_{x y}(\tau)=R_{x x}(\tau) \otimes h(\tau)=h(\tau)$

where $y(t)$ is the channel output and $R_{x x}(\tau)=\delta(\tau)$. 
Using the same technique and transmitting a pseudo-noise (PN) sequence, one can obtain an estimate of the equivalent impulse response of a radio channel. It is only an estimate since spectrum of a PN sequence is only flat in a limited bandwidth, whereas white noise has an infinite bandwidth. As the chip rate of the PN sequence, and hence its bandwidth increases, the estimation becomes more accurate.

In practice, in order to measure the impulse response, the cross-correlation can be written as:

$$
R_{x y^{\prime}}(t, \tau)=h(t, \tau) \otimes h_{s y s}(t, \tau) \otimes R_{x x}(t, \tau)
$$

Hence, the radio channel's impulse response is not only convolved with the autocorrelation of the input PN sequence $R_{x x}(t, \tau)$, but also with the impulse response of the measurement system $h_{s y s}(t, \tau)$. In our experiment, a channel sounding system was used, and the total channel is comprised of the transmitter (Tx), mobile radio channel, and the receiver $(\mathrm{Rx})$, so the impulse response estimates are defined as the system response estimates, i.e. the experimental system response estimates (abbreviated as SREs in this thesis). A Back-to-Back (BB) test in each frequency band was done to obtain a reference sequence under the condition that the channel is ideal channel and approximated by a distortionless transmission line between the system Tx and Rx. Auto correlation functions (ACFs) and transfer functions (TFs) of the $\mathrm{BB}$ reference sequences at 2 and $6 \mathrm{GHz}$ are shown in Fig.3.1.2. These were recorded when the input power at the receiver at 2 and $6 \mathrm{GHz}$ were $-10 \mathrm{dBm}$ and $-11.5 \mathrm{dBm}$, respectively. The multipath power sensitivity ratio (MPSR defined as the max power to the noise plus sidelobe level) at both frequencies is $-50 \mathrm{~dB}$. Above the threshold $-45 \mathrm{~dB}$, the delay caused by the system is the same at the two frequencies. 


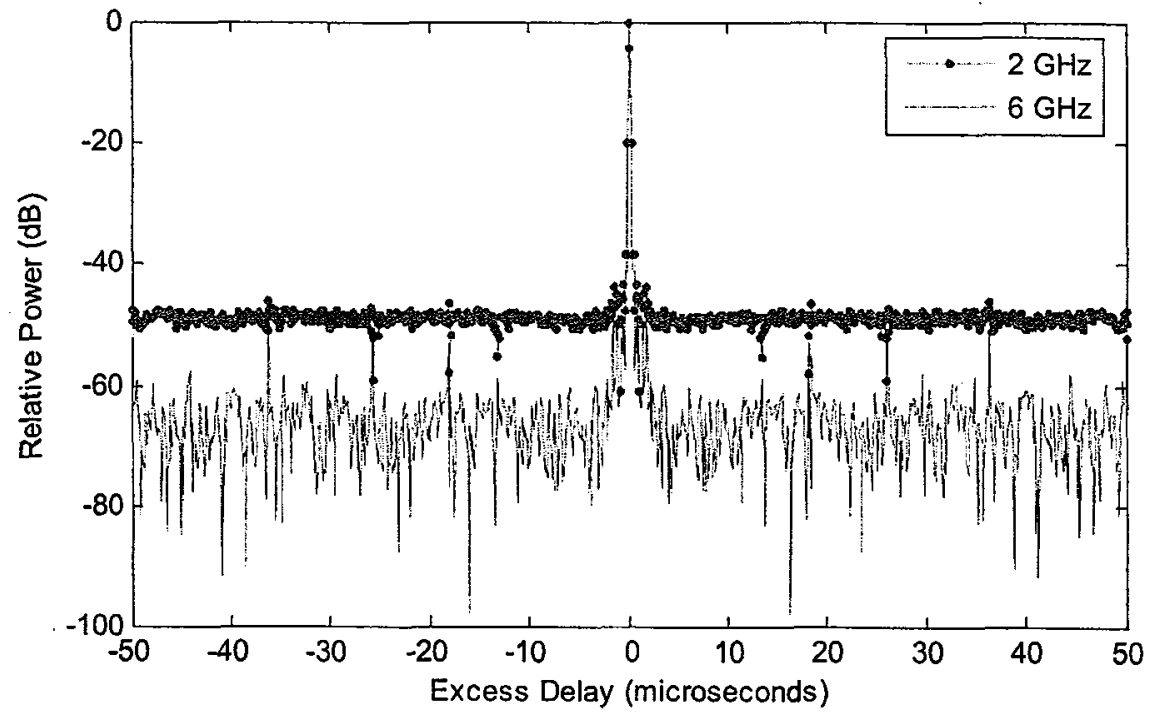

(a): Auto Correlation Functions of Back-to-Back reference sequences at 2 and $6 \mathrm{GHz}$

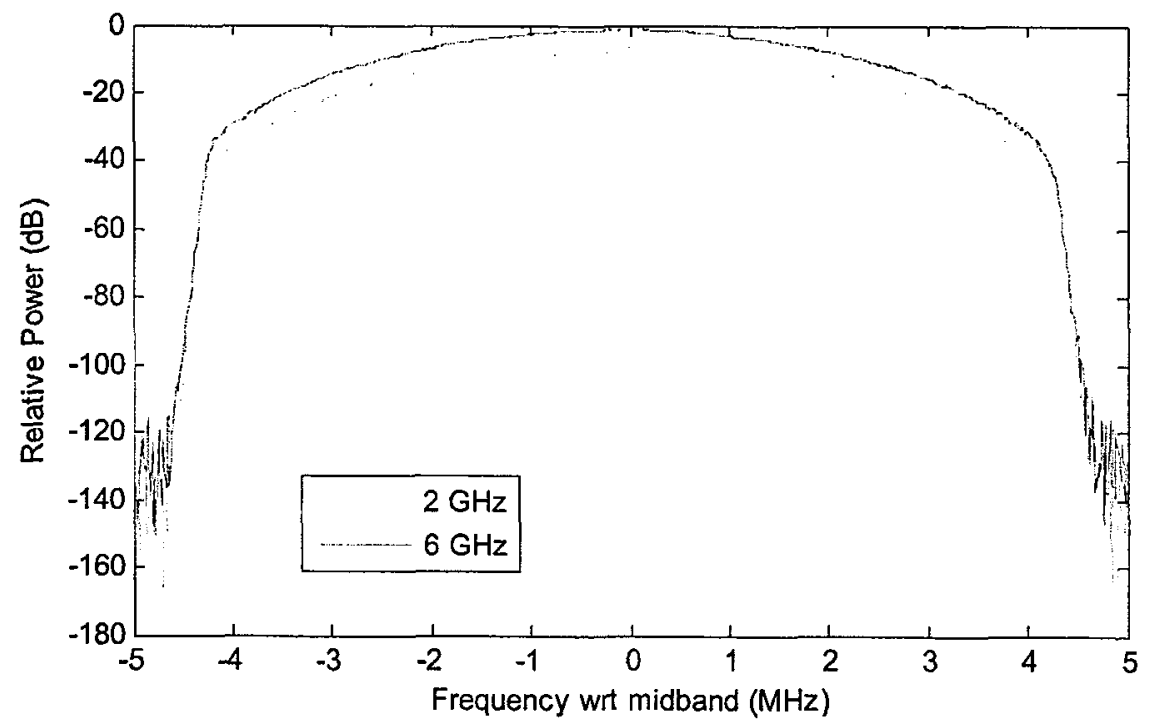

(b): Transfer Functions of Back-to-Back reference sequences at 2 and $6 \mathrm{GHz}$.

Fig.3.1.2: ACFs and TFs of BB reference sequences at 2 and $6 \mathrm{GHz}$.

During the measurement, a PN sequence of length 511 chips was transmitted at a rate of $5 \mathrm{Mchips} / \mathrm{s}$, which is very close to the chip rate of $3.84 \mathrm{Mchips} / \mathrm{s}$ used in IMT 2000 
systems. SREs estimated from such radio channel soundings are therefore similar to the equivalent channel impulse response on single links in IMT2000 systems.

According to Nyquist's sampling theorem, the minimum sampling rate should be at least twice the maximum rate of channel variations. Herein, the measurement snapshot rate should be:

$$
f_{\text {snapshot }} \geq 2 f_{D_{\max }}
$$

where $f_{D_{\max }}$ is the maximum Doppler frequency, $f_{D_{\max }}=v / \lambda$, where $v$ represents the vehicle speed and $\lambda$ represents the wavelength of the transmitted signal.

The channel snapshot rate is reciprocal of the time interval $t_{S N A P}$ between the consecutively sampled snapshots of the received PN sequences, so one can get:

$$
t_{S N A P} \leq \frac{1}{2 f_{D_{\max }}}
$$

The wavelength of the signal at $2.25 \mathrm{GHz}$ is $\lambda_{2}=\frac{c}{f_{c}}=0.1333 \mathrm{~m}$. Thus the maximum time interval between consecutively sampled snapshots should be $11.7 \mathrm{~ms}$ when the vehicle speed is, say, $20 \mathrm{Km} / \mathrm{h}$. At $5.8 \mathrm{GHz}, \lambda_{6}=0.0517 \mathrm{~m}$, and the maximum time interval between consecutively sampled snapshots at this frequency should be $4.5 \mathrm{~ms}$ when the vehicle speed is $20 \mathrm{Km} / \mathrm{h}$.

In the work of this thesis, there are three sets of measured data. One set of data was measured and recorded separately on different days at $1.9 \mathrm{GHz}$ and $5.8 \mathrm{GHz}$ in downtown Ottawa by CRC before this thesis project began, we will refer to this as the old set of data. The other two sets of data were measured and recorded quasi-simultaneously 
(i.e. within 20 microseconds of each other) at $2.25 \mathrm{GHz}$ and $5.8 \mathrm{GHz}$ in downtown Ottawa during the work of this thesis, we refer to this as the new sets of data. For convenience in the later data analysis, 1.9 and $2.25 \mathrm{GHz}$ work is referred to as being at 2 $\mathrm{GHz}$, and $5.8 \mathrm{GHz}$ work is referred to as being at $6 \mathrm{GHz}$ (i.e. in the 2 and $6 \mathrm{GHz}$ frequency bands, respectively).

The old set of data was recorded with a snapshot rate of $450 \mathrm{~Hz}$, i.e. $2.2 \mathrm{~ms}$ time interval between consecutively sampled snapshots, while the new sets of data were recorded quasi-simultaneously with a snapshot rate of $750 \mathrm{~Hz}$, i.e. $1.3 \mathrm{~ms}$ time interval between consecutively sampled snapshots.

The snapshot rate of $450 \mathrm{~Hz}$ is more than 10 times of the Doppler spread of $2 \mathrm{GHz}$ $\left(f_{D_{\operatorname{mix}}}=42.7 \mathrm{~Hz}\right)$, and 4 times of the Doppler spread of $6 \mathrm{GHz}\left(f_{D_{\max }}=111.1 \mathrm{~Hz}\right)$ at the maximum measurement vehicle speed of $20 \mathrm{~km} / \mathrm{h}$, which was limited to this value as a reasonable tradeoff between sampling rate and memory space considerations.

The measurement system consists of a pseudo-noise (PN) coded, BPSK Tx and a direct sampling Rx. Sampled data are stored during field work, and later cross correlated with a BB PN reference sequence that was recorded when the $\mathrm{Tx}$ and $\mathrm{Rx}$ were connected via a short coaxial cable.

\subsubsection{The Transmitter}

The measurement system (referred to as CRC-Chanprobe) Transmitter block diagram is shown in Fig.3.1.3. In the Transmitter, a PN generator consisting of feedback shift registers generates 511 chip sequences. The 5 Mchips/s chip clock is generated by a digital PLL. Hence, a delay resolution of $200 \mathrm{~ns}$ is achieved with a $10 \mathrm{MHz}$ RF 
bandwidth. PN sequences are modulated on a $950 \mathrm{MHz}$ carrier. After a band pass filter, a power splitter splits the power of the $950 \mathrm{MHz}$ modulated signal and applies it to different up converter / power amplifier / antenna chains. The transmitter simultaneously transmits two BPSK PN modulated signals, one at $2.25 \mathrm{GHz}$ and one at $5.8 \mathrm{GHz}$.

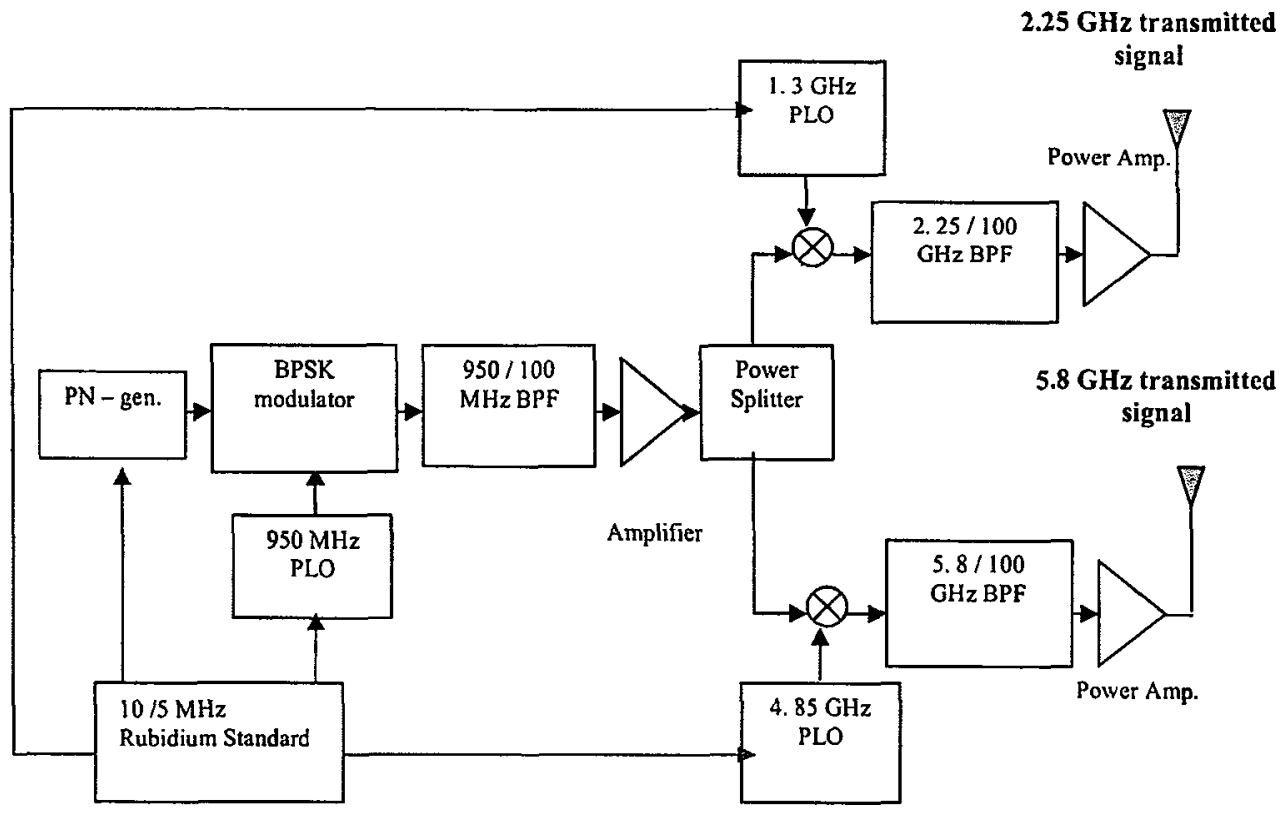

Fig.3.1.3: Measurement system Transmitter block diagram.

\subsubsection{The Receiver}

One broadband biconical antenna is used to receive both 2.25 and $5.8 \mathrm{GHz}$ signals. Fig.3.1.4 shows the receiver block diagram. Through the control of a PC, the switch near the receiver front end can be used to sequentially receive in both 2 and $6 \mathrm{GHz}$ frequency bands.

In the Receiver, the received signals pass through 2.25 and $5.8 \mathrm{GHz}$ band pass filters. Using a mixer, the $5.8 \mathrm{GHz}$ signal is first down converted to $2.25 \mathrm{GHz}$. A switch is used 
to automatically switch signals at 2.25 and $5.8 \mathrm{GHz}$. A programmable attenuator is then used to adjust the power level to avoid clipping at the baseband input to the A / D converter. After a second $2.25 \mathrm{GHz}$ band pass filter and amplifier, the signal is down converted to a $70 \mathrm{MHz}$ IF. After a $70 \mathrm{MHz}$ band pass filter, amplifier and I / Q demodulator, the baseband I / Q signals can be sampled under PC control using a 16-bit A/D converter. Multiple conversion stages are used to facilitate the use of the measurement system for different experiments with different bandwidths at different frequencies.

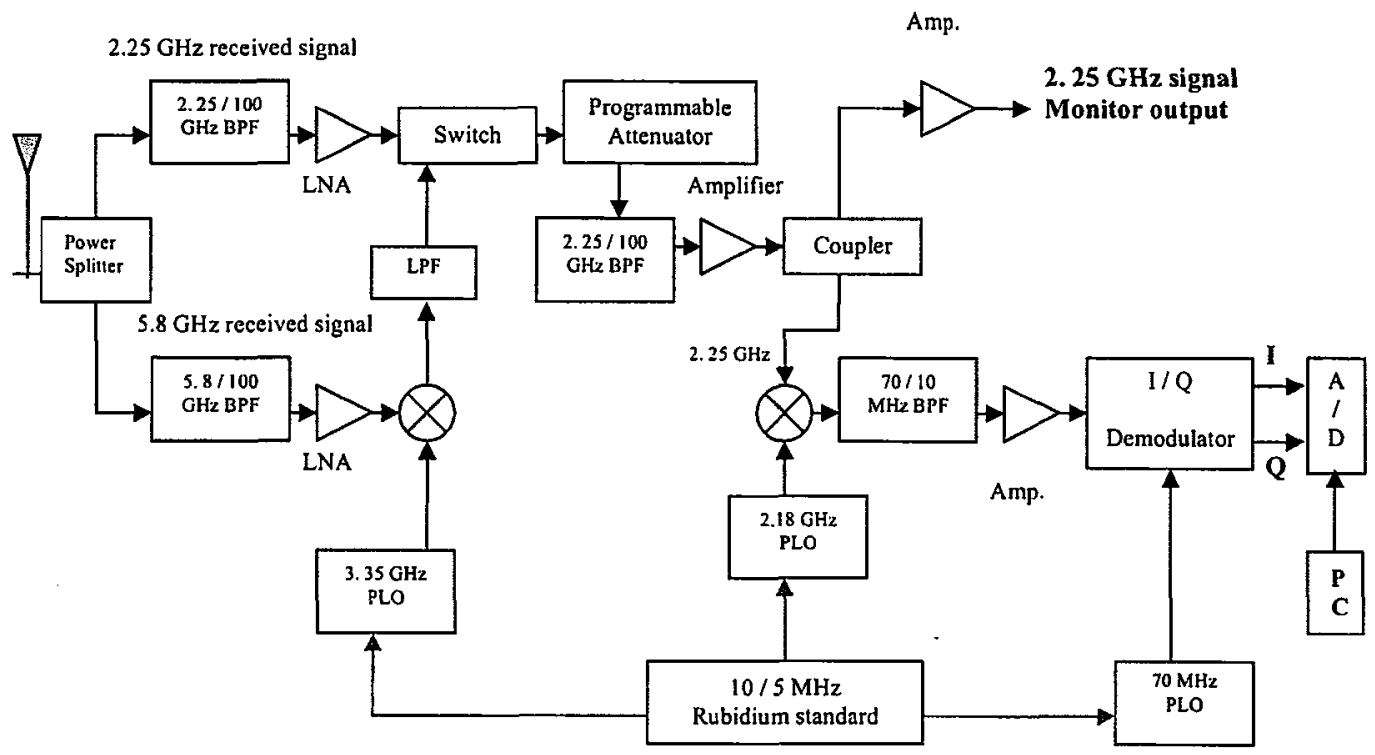

Fig. 3.1.4: Measurement system Receiver block diagram. 


\subsubsection{System calibration tests}

In order to determine the region of linear operation for the $\mathrm{Rx}, \mathrm{BB}$ calibration tests for the measurement system are important. An HP variable attenuator was used to adjust the input power level at the Receiver front end, as shown in Fig.3.1.5.

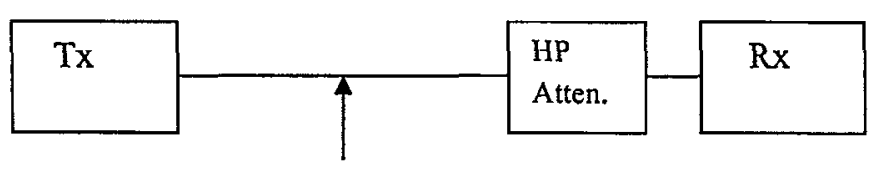

Short coaxial cable

Fig.3.1.5: BB calibration tests configuration.

\subsubsection{2.25 GHz calibration tests}

Before the Tx power amplifier, which was omitted from the BB tests, the power of the Tx signal at $2.25 \mathrm{GHz}$ was $-10 \mathrm{dBm}$. After setting the $\mathrm{HP}$ attenuator to $6 \mathrm{~dB}$, and adding two $10 \mathrm{~dB}$ fixed pads, the input power to the $\mathrm{Rx}$ front end was measured to be $-36 \mathrm{dBm}$. Then when the programmable attenuator was set to $38 \mathrm{~dB}$, an oscilloscope indicated the reception of a PN signal with one-volt peak amplitude at the A/D converter input, which is specified to operate without clipping at that level. A power meter at the $2.25 \mathrm{GHz}$ monitor output showed $-8 \mathrm{dBm}$.

The above-described $\mathrm{BB}$ recording was used as a $\mathrm{BB}$ reference to obtain later cross correlation results.

Increasing the $\mathrm{HP}$ attenuator to $10 \mathrm{~dB}$, the $\mathrm{Rx}$ input power was changed to $-40 \mathrm{dBm}$. Decreasing the programmable attenuator from $38 \mathrm{~dB}$ to $34 \mathrm{~dB}$ to compensate for the $4 \mathrm{~dB}$ loss, the power of the $2.25 \mathrm{GHz}$ monitor output was kept at $-8 \mathrm{dBm}$. 
The $2^{\text {nd }} \mathrm{BB}$ recording was taken and indicated that the ACF and the cross correlation functions (CCF) were symmetrical and clean with MPSRs 44 and $46 \mathrm{~dB}$, respectively. The TF was symmetrical and very clean.

Increasing the $\mathrm{HP}$ attenuator by another $5 \mathrm{~dB}$ to $15 \mathrm{~dB}$, the $\mathrm{Rx}$ input power was changed to $-45 \mathrm{dBm}$. Decreasing the programmable attenuator to $29 \mathrm{~dB}$ to compensate for the $5 \mathrm{~dB}$ loss, the power of the $2.25 \mathrm{GHz}$ monitor output was kept at $-8 \mathrm{dBm}$.

The $3^{\text {rd }} \mathrm{BB}$ recording was taken and showed that the $\mathrm{ACF}$ and $\mathrm{CCF}$ were symmetrical and clean with MPSRs 44 and $43 \mathrm{~dB}$, respectively. CCF with ears was at 40 $\mathrm{dB}$. The TF was symmetrical and very clean.

Step by step a total of $17 \mathrm{BB}$ test files were recorded at $2.25 \mathrm{GHz}$. The CCF peak powers as a function of relative input power in front of the $\mathrm{Rx}$ are shown in Fig.3.1.6. It can be seen that the linear region of input power for the $\mathrm{Rx}$ was from $-75 \mathrm{dBm}$ to -115 $\mathrm{dBm}$. During field measurements, when received power was greater than $-75 \mathrm{dBm}$, as indicated by clipping of recorded test signals, either an attenuator at the $T x$ or the programmable attenuator in the $\mathrm{Rx}$ was adjusted so as to maintain the input to the $\mathrm{A} / \mathrm{D}$ converter to be less than one-volt peak.

\subsubsection{2 $5.8 \mathrm{GHz}$ calibration tests}

Identical calibration tests were conducted at $5.8 \mathrm{GHz}$. Results are shown along with the $2.25 \mathrm{GHz}$ results in Fig.3.1.6. It can be seen that the linear region of the input power for the $\mathrm{Rx}$ without power amplifier in $\mathrm{Tx}$ operated at $5.8 \mathrm{GHz}$ was from $-70 \mathrm{dBm}$ to -115 $\mathrm{dBm}$. 


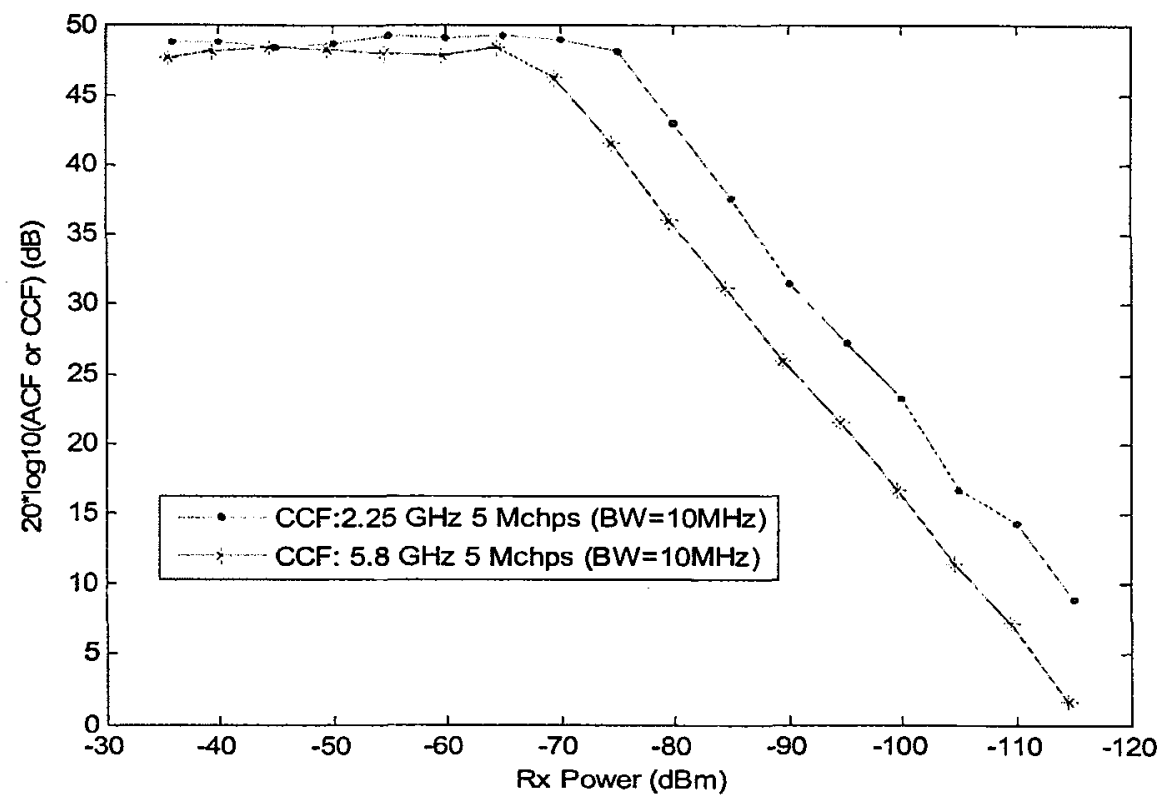

Fig.3.1.6: BB calibration test results at $2.25 \mathrm{GHz}$ and $5.8 \mathrm{GHz}$.

\subsubsection{Measurement setup}

Quasi-simultaneous 2 and $6 \mathrm{GHz}$ field measurements were conducted in downtown Ottawa. To simulate a microcellular base-station, the $\mathrm{Tx}$ was housed in a van parked at a curbside. PN sequences of 511 chips in length were transmitted at a rate of $5 \mathrm{Mchips} / \mathrm{s}$. After BPSK modulation and up-conversion, the 2 and $6 \mathrm{GHz}$ band RF signals were transmitted through different antennas, which were mounted on a mast above the van with a height of $5.5 \mathrm{~m}$ above ground level.

The Rx was in a minivan that was driven at traffic speeds less than $20 \mathrm{~km} / \mathrm{h}$ on all streets within about 4 city blocks of the Tx site. A single biconical antenna was mounted on the roof of the minivan and used to receive in both frequency bands. The A/D converter output was sampled at $10 \mathrm{Msamples} / \mathrm{second}$ (the sample period is $100 \mathrm{~ns}$ ) and 
recorded. During each measurement snapshot, one sequence length was recorded in each frequency band.

Both the $\mathrm{Tx}$ and $\mathrm{Rx}$ oscillators in the measurement system were slaved to phasecoherent rubidium standards and all clocks ran synchronously. This rendered recorded data that were suitable for later time-series analyses.

Cross correlations between the measurement data recorded in the field and the laboratory-recorded $\mathrm{BB}$ PN reference sequence resulted in the SREs that were used in all data analyses. 


\subsection{Data analyses}

This section describes the data analysis methods used for the estimation of channel parameters. The analysis results including a comparison of channel characteristics in the 2 and $6 \mathrm{GHz}$ frequency bands are also presented.

\subsubsection{Analysis methods}

The radio channel measurement system is a DSSS Tx / Rx system with a $10 \mathrm{MHz} R F$ bandwidth. This renders approximately the same delay resolution as an IMT2000 receiver. The methods of obtaining received signal of interest are presented in the following section.

\subsubsection{Average Power Delay Profile (APDP) and Multipath Group (MPG) identification}

An APDP, which is the expected value of the received power at a certain delay, is the basic parameter to characterise the significance of delay dispersion on the channel and multipath effects. Herein for each street section (referred to below as a "channel") where propagation measurements were made, an APDP is the result from averaging of the squared magnitude of consecutive SREs in 1-sec-long time series.

Through the obtained APDP, one can easily find the strongest MPG of the channel. The second strongest MPG was selected based the consideration that adjacent samples from the measurement system are really within the resolution bandwidth of the $\mathrm{Rx}$, so would be expected to exhibit highly correlated characteristics. Thus, in this thesis, 
adjacent MPGs were selected as being spaced apart by 3 samples or at 1.5 chip intervals, since with greater spacings, power falls off too rapidly.

Fig.3.2.1 shows the APDP which was obtained from the old set of data measured on a typical street section: Bank Street, between Gloucester St. and Laurier Avenue.
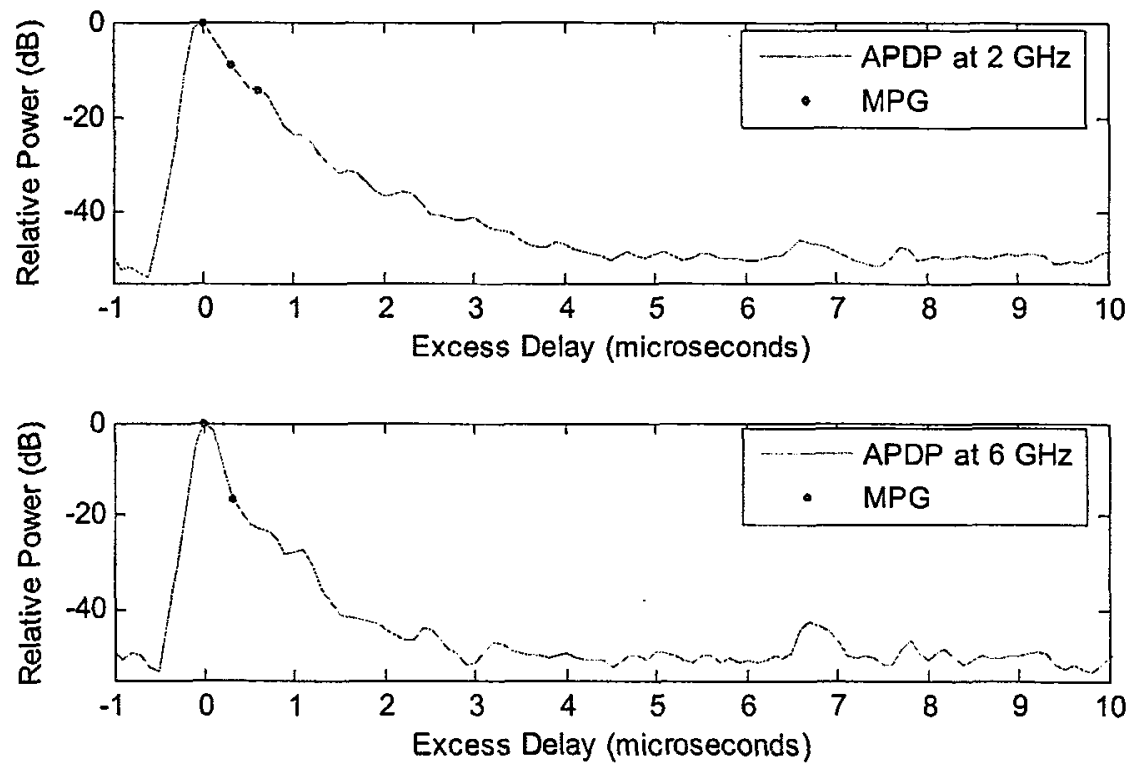

Fig.3.2.1: APDP and MPGs estimated from the old set of data recorded on Bank Street: between Gloucester St. and Laurier Ave.

The APDPs were obtained by averaging the squared magnitudes of 1-sec-long consecutive SREs in time series at 2 and $6 \mathrm{GHz}$ for the same street section. The MPGs of interest in the APDPs were those, which had powers relative to the main peak in the APDPs of greater than $-20 \mathrm{~dB}$. As mentioned previously, the MPGs were picked with 1.5 chip intervals. There are three MPGs at $2 \mathrm{GHz}$ and $2 \mathrm{MPGs}$ at $6 \mathrm{GHz}$ on the above typical links. 
Another example in Fig.3.2.2 shows the APDP obtained from the measured data recorded on Kent Street, between Slater St. and Albert St. Above the threshold $-20 \mathrm{~dB}$, there were 5 received MPGs at $2 \mathrm{GHz}$ with relative powers $0,-9.8 \mathrm{~dB},-11.1 \mathrm{~dB},-14.5 \mathrm{~dB}$ and $-18.3 \mathrm{~dB} ; 6$ received MPGs at $6 \mathrm{GHz}$ with relative powers $0,-3.5 \mathrm{~dB},-6.7 \mathrm{~dB},-10.8$ $\mathrm{dB},-16.7 \mathrm{~dB}$ and $-19.6 \mathrm{~dB}$.
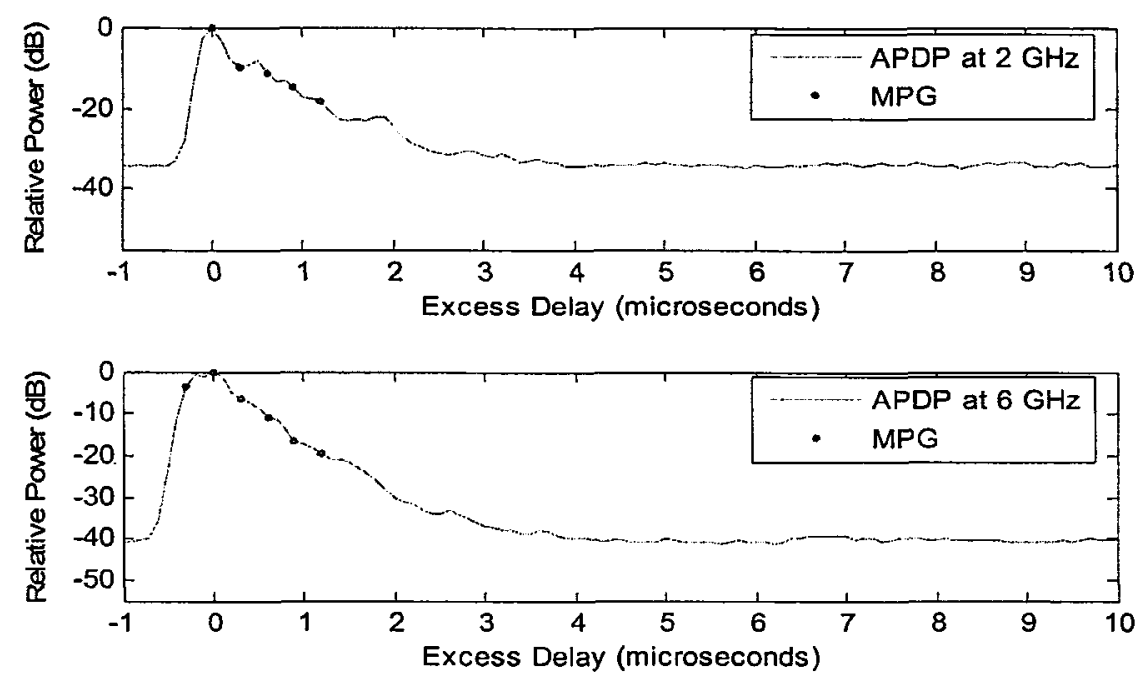

Fig.3.2.2: APDP and MPGs estimated from the old set of data recorded on Kent Street: between Slater St. and Albert St.

The time series of envelope fading for the three strongest MPGs at 2 and $6 \mathrm{GHz}$ are shown in Fig.3.2.3 and Fig.3.2.4, respectively. The figures show that $6 \mathrm{GHz}$ data presents more fading compared with $2 \mathrm{GHz}$ data within the same 1-sec-long snapshot. That is predictable because at high frequency, the phase of the signal changes faster resulting in more fading compared with the fading of a signal at a lower frequency. 


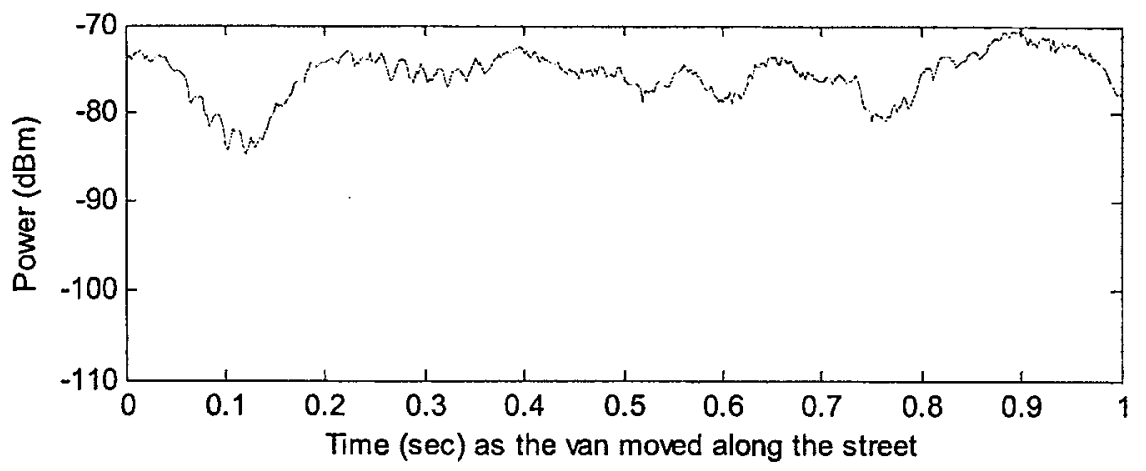

(a) Envelope time series of the strongest MPG

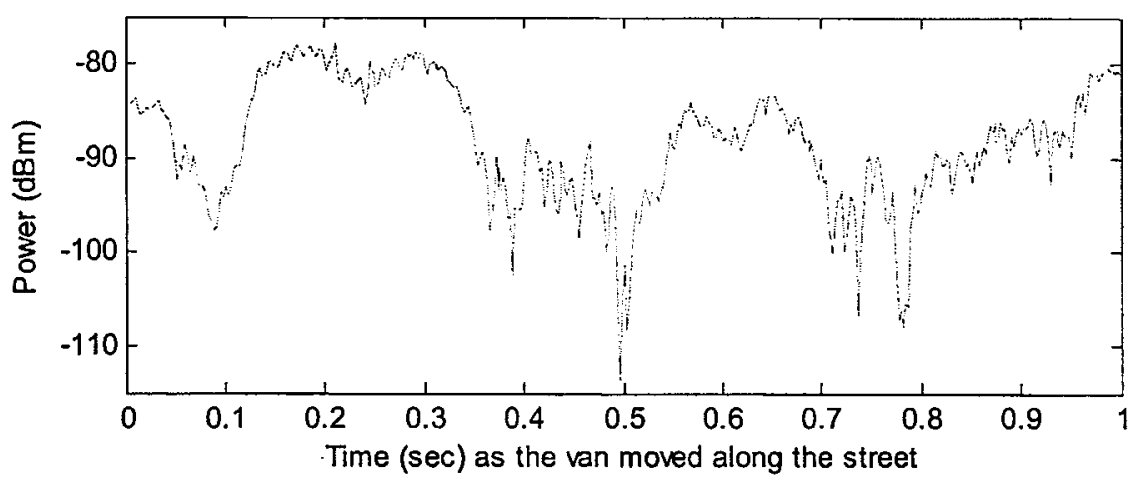

(b) Envelope time series of the second strongest MPG

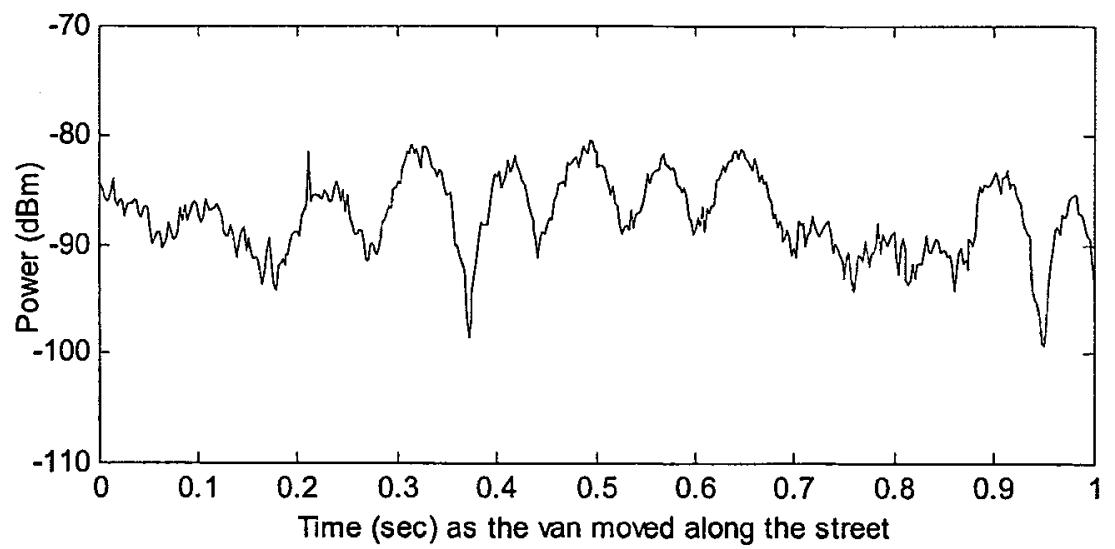

(c) Envelope time series of the third strongest MPG

Fig.3.2.3: Envelope time series of the three strongest MPGs at $2 \mathrm{GHz}$ on Kent Street, between Slater St. and Albert St. 


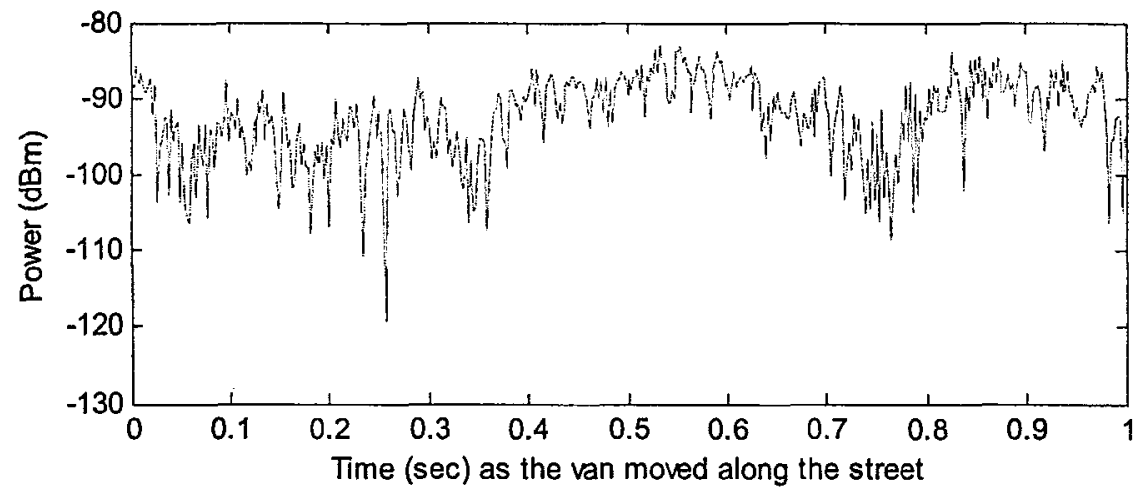

(a) Envelope time series of the strongest MPG

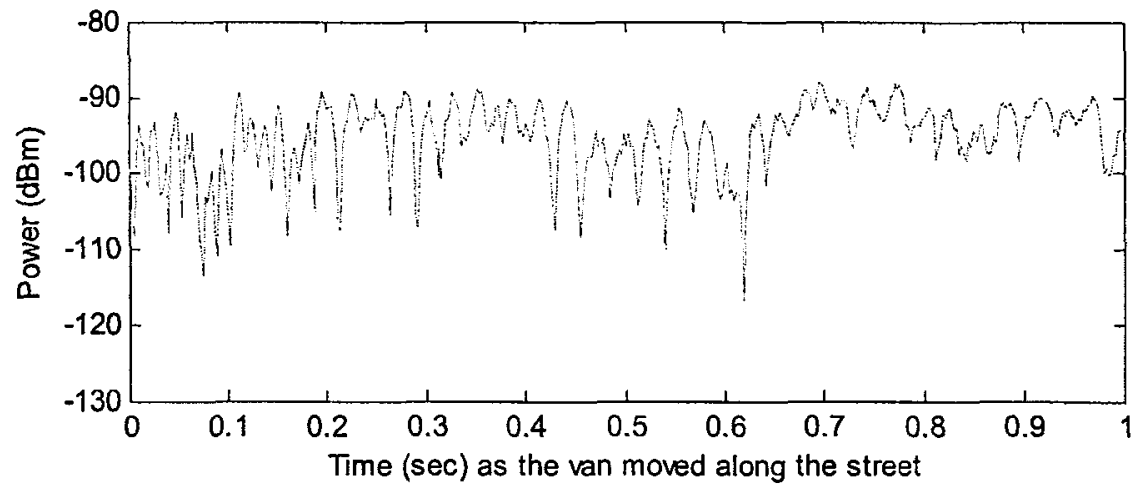

(b) Envelope time series of the second strongest MPG

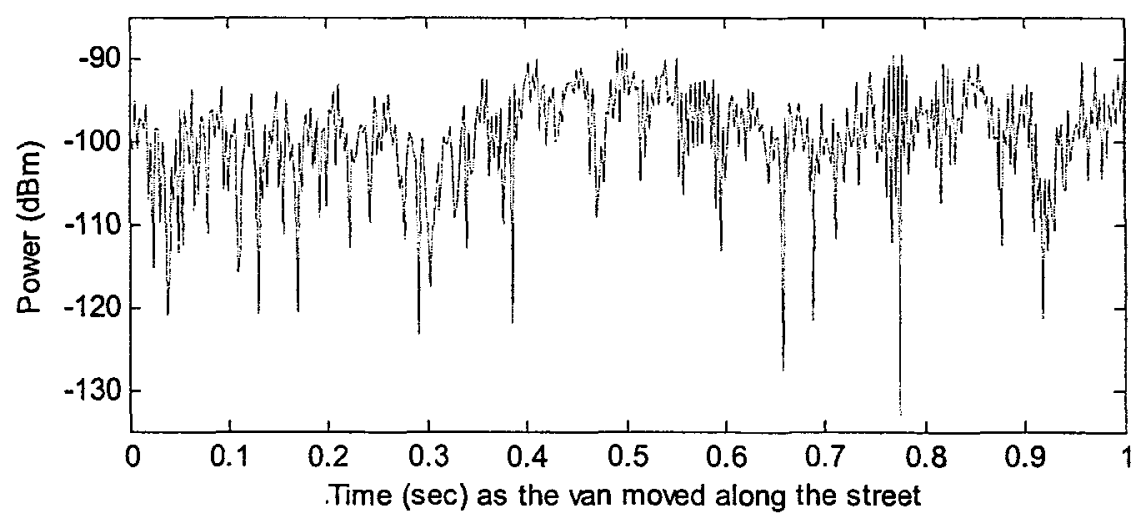

(c) Envelope time series of the third strongest MPG

Fig.3.2.4: Envelope time series of the three strongest MPGs at $6 \mathrm{GHz}$ on Kent Street, between Slater St. and Albert St. 
Experimentally determined cumulative distribution functions (ECDFs) of envelope fading for the three strongest selected MPGs at 2 and $6 \mathrm{GHz}$ are shown in Fig.3.2.5 to Fig.3.2.6.

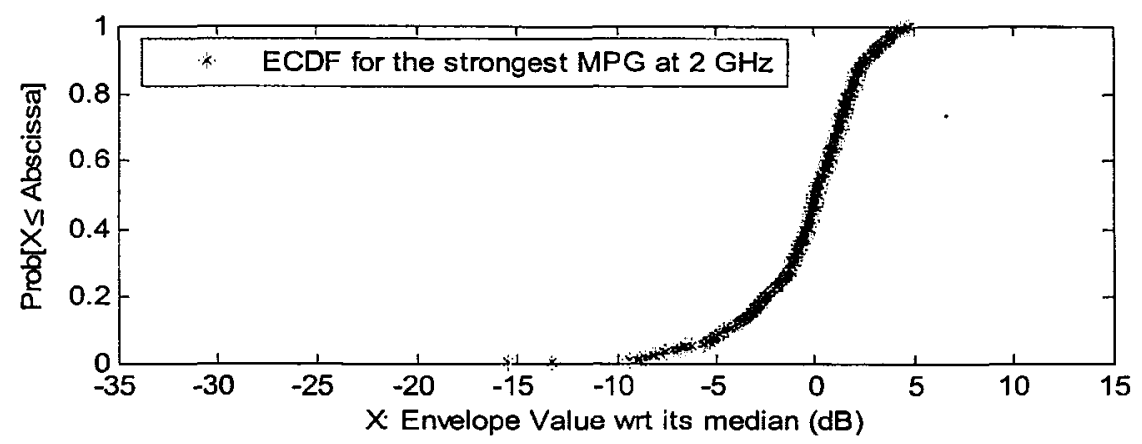

(a) ECDF of envelope fading for the strongest MPG

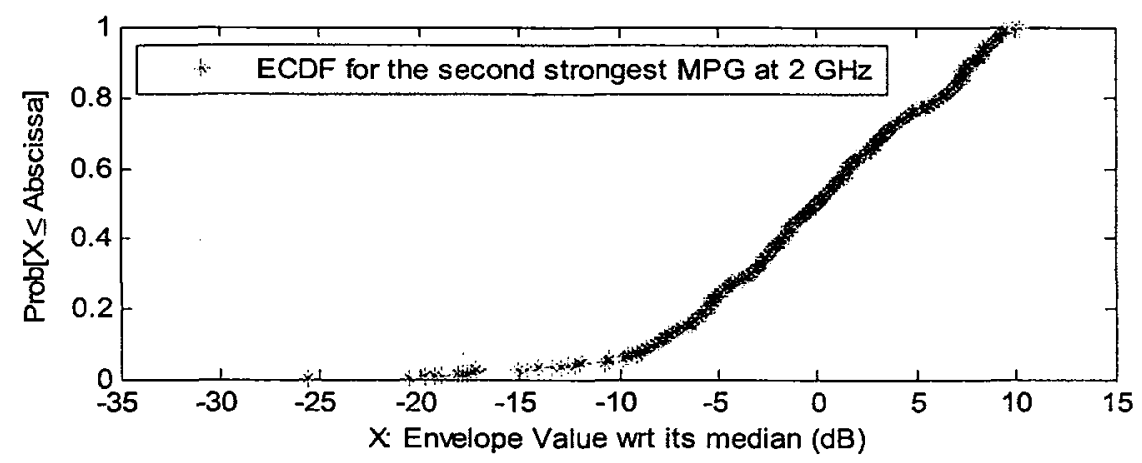

(b) ECDF of envelope fading for the second strongest MPG

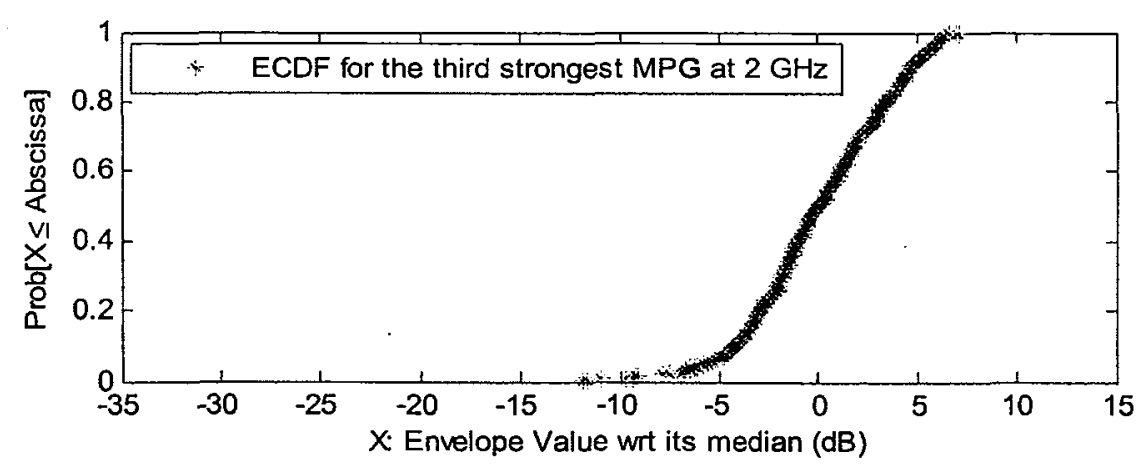

(c) ECDF of envelope fading for the third strongest MPG

Fig.3.2.5: ECDFs of envelope fading for the three strongest MPGs at $2 \mathrm{GHz}$ on Kent Street, between Slater St. and Albert St. 


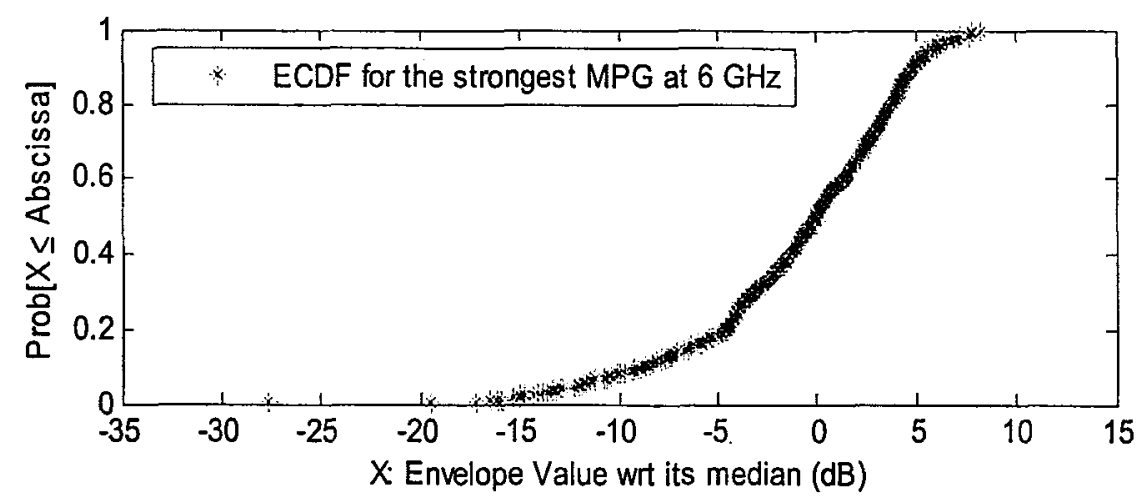

(a) ECDF of envelope fading for the strongest MPG

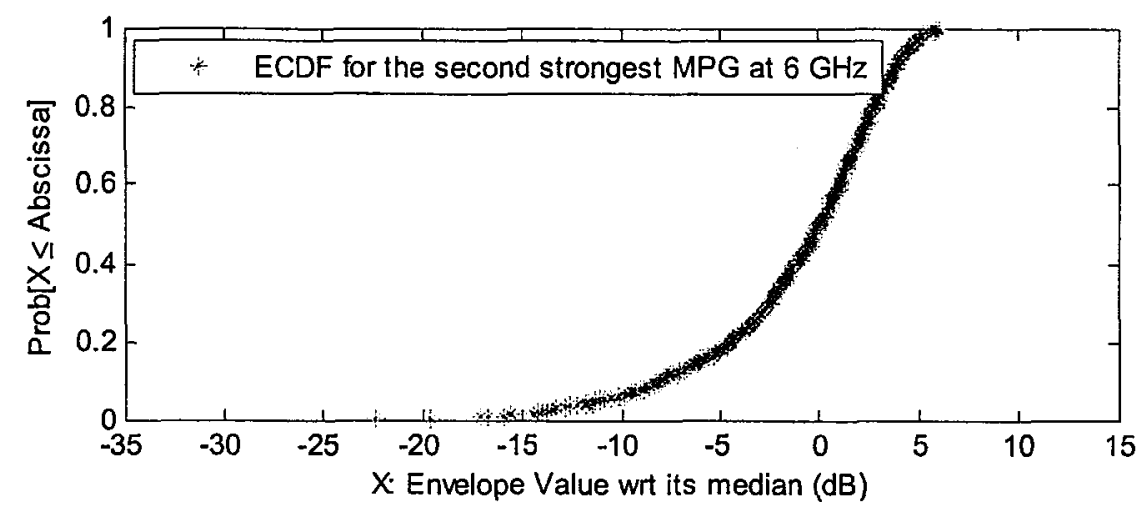

(b) ECDF of envelope fading for the second strongest MPG

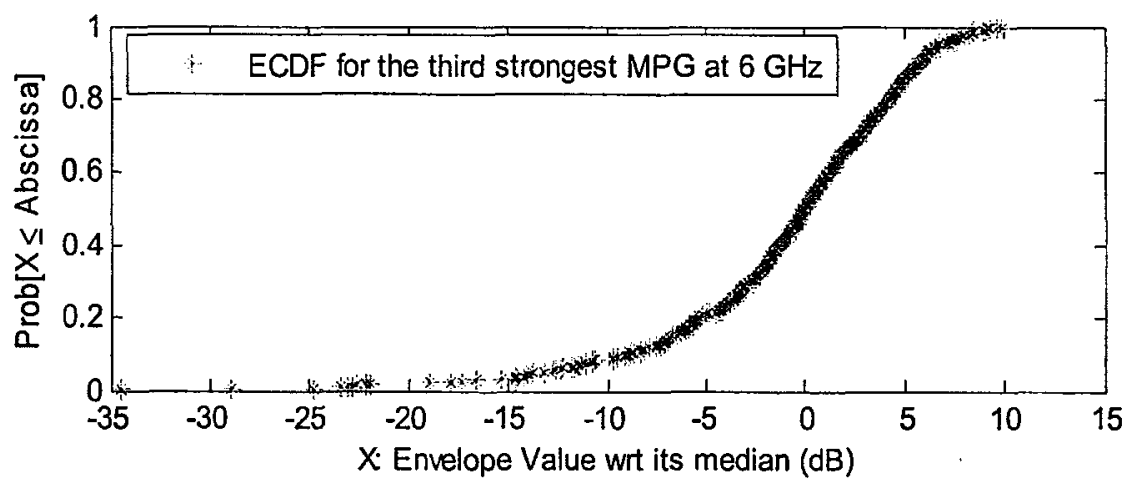

(c) ECDF of envelope fading for the third strongest MPG

Fig.3.2.6: ECDFs of envelope fading for the three strongest MPGs at $6 \mathrm{GHz}$ on Kent Street, between Slater St. and Albert St. 
One parameter, fading range (FR), is used to characterize the fading of selected MPG. Fading range is defined as the range of ECDF of envelope fading for MPG, from $10 \%$ to $90 \%$ due to unstable tails of ECDF under the conditions of limited measured data samples in each case. The fading ranges for the above three strongest MPGs at $2 \mathrm{GHz}$ were thus determined to be $7 \mathrm{~dB}, 15 \mathrm{~dB}$ and $9 \mathrm{~dB}$. The fading ranges of those at $6 \mathrm{GHz}$ were determined to be $13 \mathrm{~dB}, 12 \mathrm{~dB}$ and $13 \mathrm{~dB}$. It should be noted that the greater values at $6 \mathrm{GHz}$ reflect a greater probability of fading rather than deeper fade depths. Cross correlation between fading envelopes of the selected MPGs were calculated. For the above case, the correlation coefficient matrices of envelope fading for the three strongest MPGs at 2 and $6 \mathrm{GHz}$ were evaluated and the results show that the correlation coefficients were very small, being within the range of \pm 0.2 .

$$
\left\{\begin{array}{ccc}
1.0000 & 0.1070 & -0.0205 \\
0.1070 & 1.0000 & 0.0602 \\
-0.0205 & 0.0602 & 1.0000
\end{array}\right\} \text { at } 2 \mathrm{GHz} \text { and }\left\{\begin{array}{ccc}
1.0000 & 0.1385 & 0.1507 \\
0.1385 & 1.0000 & 0.0502 \\
0.1507 & 0.0502 & 1.0000
\end{array}\right\} \text { at } 6 \mathrm{GHz}
$$

The above result indicates that the selected MPGs exhibited negligible correlation and should be suitable for use in Rake combining. The $-7 \mathrm{~dB}$ is the lowest relative power to the strongest MPG for which worthwhile combining gains can be achieved when there are channel estimation errors. On the above typical radio links, three MPGs whose powers were greater than $-7 \mathrm{~dB}$ were used for Rake combining at $6 \mathrm{GHz}$, while only the strongest MPG was used at $2 \mathrm{GHz}$. Fig.3.2.7 shows the comparison of time series and ECDF of fading envelope after Rake combining at $6 \mathrm{GHz}$ and those for the strongest MPG at $2 \mathrm{GHz}$. From the figure, one can get the fading range of the ECDF after Rake combining at $6 \mathrm{GHz}$ is $7 \mathrm{~dB}$, same as that of the strongest MPG at $2 \mathrm{GHz}$. The analysis of BER performance on this typical channel will be shown in Chapter 4. 


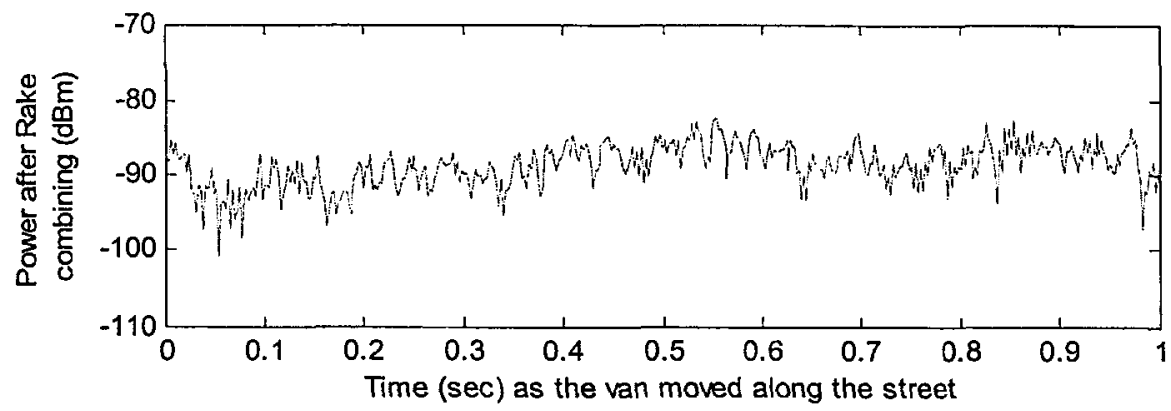

(a) Time series of envelope fading after Rake combining at $6 \mathrm{GHz}$

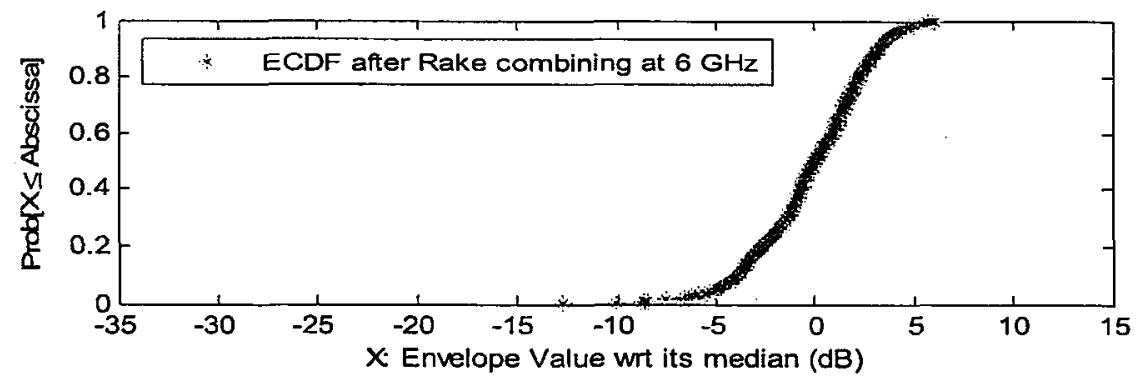

(b) ECDF of envelope fading after Rake combining at $6 \mathrm{GHz}$

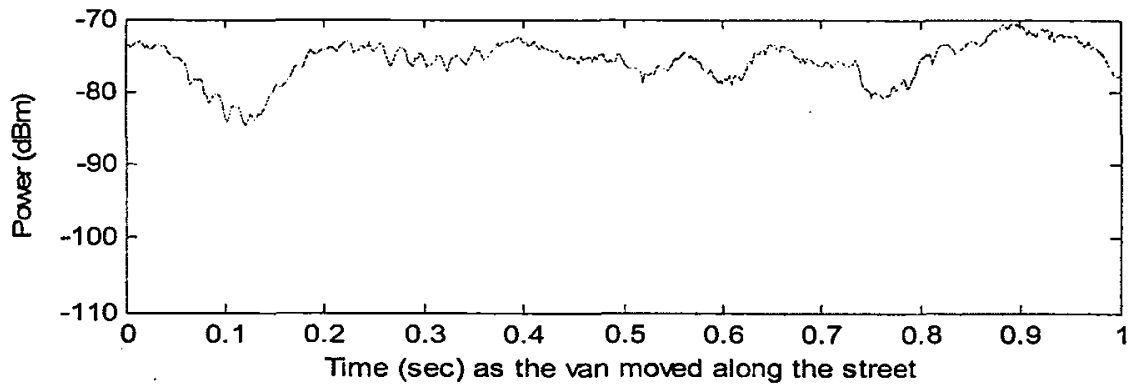

(c) Time series of envelope fading for the strongest MPG at $2 \mathrm{GHz}$

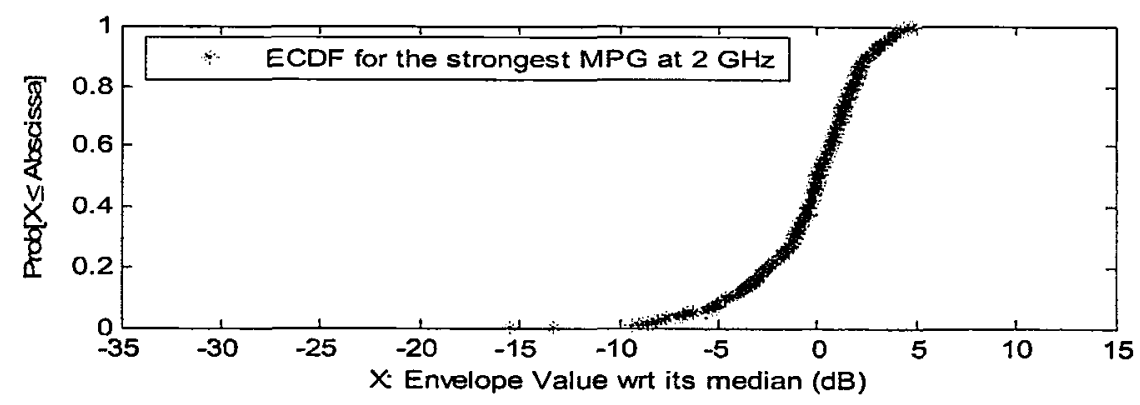

(d) ECDF of envelope fading for the strongest MPG at $2 \mathrm{GHz}$

Fig.3.2.7: Comparison of time series and ECDF of envelope fading after Rake combining of the three strongest MPGs at $6 \mathrm{GHz}$ and those for the strongest MPG at $2 \mathrm{GHz}$ on Kent Street, between Slater St. and Albert St. 


\subsubsection{RMS delay spreads}

The RMS delay spread associated with a fading radio channel is the square root of the second central moment of the APDP and can be written as $\sigma_{\tau}=\sqrt{\overline{\tau^{2}}-(\bar{\tau})^{2}}$.

where $\bar{\tau}=\frac{\sum_{k} P\left(\tau_{k}\right) \tau_{k}}{\sum_{k} P\left(\tau_{k}\right)}$ is the mean excess delay and $\overline{\tau^{2}}=\frac{\sum_{k} P\left(\tau_{k}\right) \tau_{k}^{2}}{\sum_{k} P\left(\tau_{k}\right)}$, where $P\left(\tau_{k}\right)$ is the average power received at delay $\tau_{k}$.

However, there are practical concerns in analysing mobile radio data, such as the tracking of time varying delays of received multipath components, and the presence of sounder signal cross-correlation sidelobes and receiver noise. The methods that were used for dealing with these problems in estimating RMS delay spreads from the old set of data were different from the methods applied to analyse the data that were newly recorded for the thesis project reported herein.

The old set of data recorded previously was somewhat noisier, and they also contained artifacts that were probably the result of clipping by the $A / D$ converter in CRC's previous measurement system's data collection system, constructed in 1992 and retired in 1998. Two steps were taken to post process these older data before estimating RMS delay spreads: First, all APDPs were framed within a window with a reasonable width. The width of the window was set $12 \mathrm{~ms}$ because a consideration of physics (this is propagation loss) shows that multipath echoes with $12 \mathrm{~ms}$ would not have relative powers as large as some of the spikes in the measured data. The energy outside this window was considered to be noise or the result of imperfections in the measurement system, so was 
excluded from the calculation. The window width was the same at 2 and $6 \mathrm{GHz}$ for all radio channels.

Second, a relative power threshold was selected. The energy lower than the threshold was considered to be noise, and was not included for the calculation. Such thresholds were different for different street sections, in accordance with the variation in received signal to noise ratio from location-to-location. The energy above the threshold was used to calculate RMS delay spreads, as shown in Fig.3.2.8.
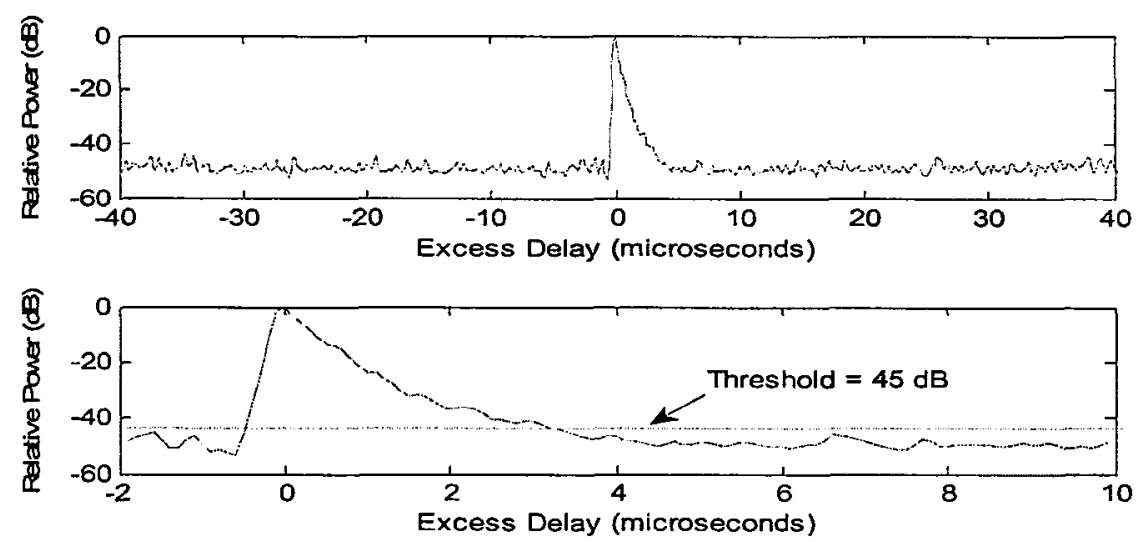

(a) RMS delay spread estimated at $2 \mathrm{GHz}$ ( $209 \mathrm{~ns}$ )
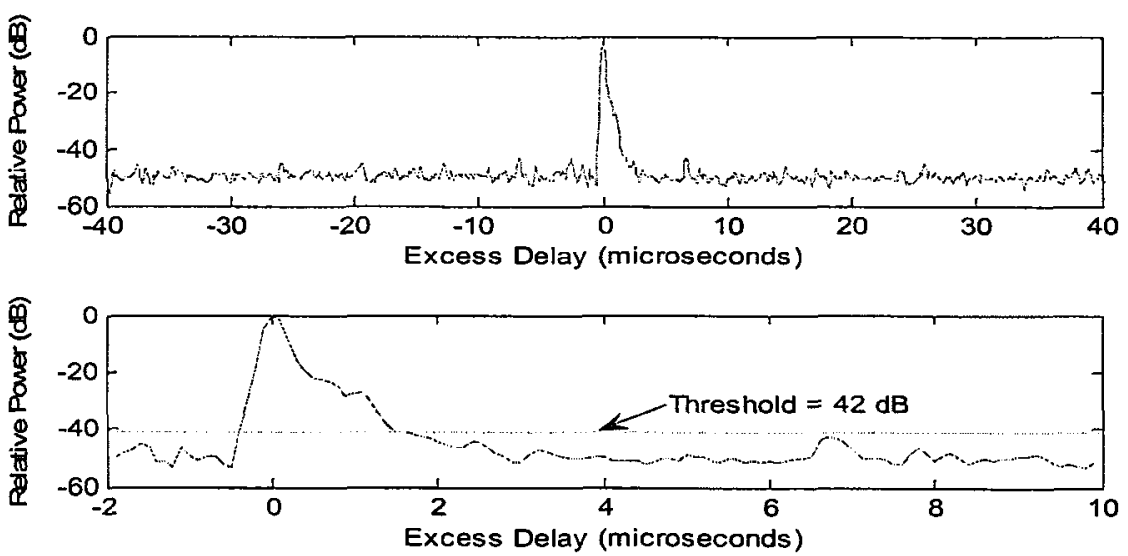

(b) RMS delay spread estimated at $6 \mathrm{GHz}(120 \mathrm{~ns})$

Fig.3.2.8: RMS delay spread from the old set of data at $2 \mathrm{GHz}$ on Bank Street, between Gloucester St. and Laurier Ave. 


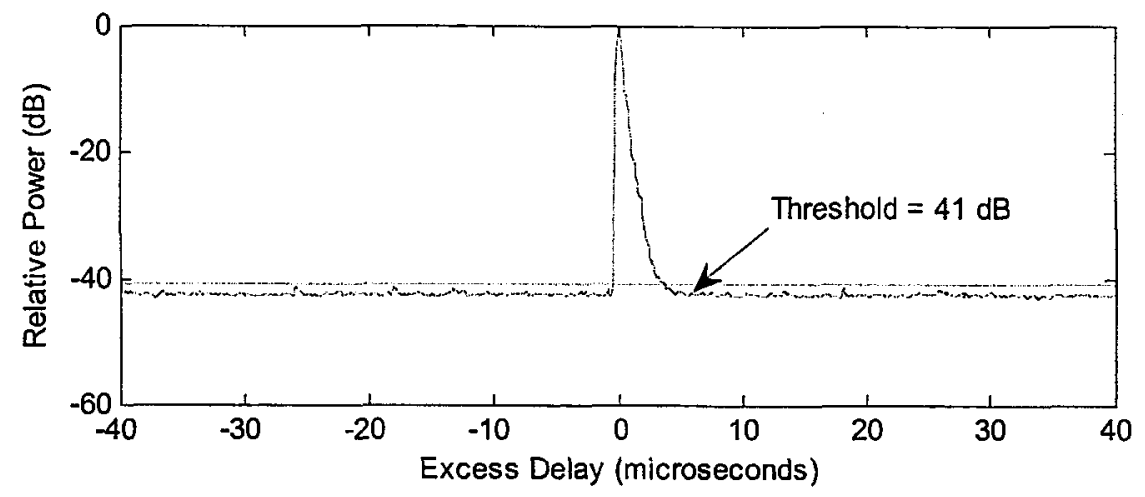

(a) RMS delay spread estimated at $2 \mathrm{GHz}$ (266 ns)

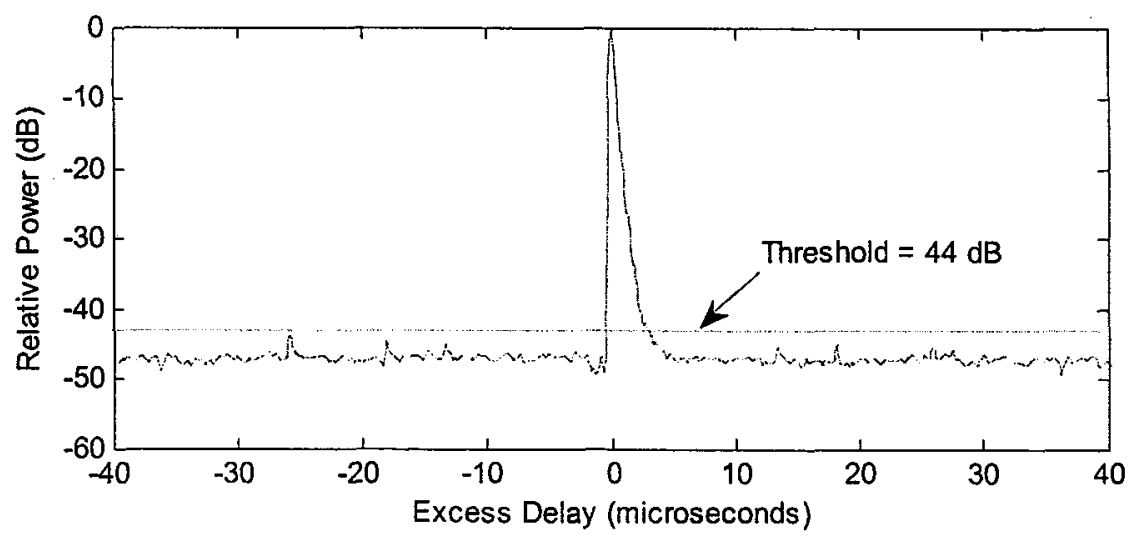

(b) RMS delay spread estimated at $6 \mathrm{GHz}$ (207 ns)

Fig.3.2.9: RMS delay spread estimated from the new set of data recorded on Kent Street, between Laurier Ave. and Slater St. with Tx on Laurier Ave.

The new set of data recorded by the quasi-simultaneous measurement system had less noise and care was taken to avoid $\mathrm{A} / \mathrm{D}$ clipping during experiments. Windowing was therefore not applied, but the noise thresholding procedure was identical. 


\subsubsection{Analysis results}

Three sets of measured data were analysed in the work of this thesis. The old set of data was collected by CRC in downtown Ottawa before the current thesis project began. Those data were measured at 2 and $6 \mathrm{GHz}$ separately on different day with the Tx at site $\mathrm{Tx} 1^{2}$ at curbside on a street that ran in an east-west direction. The two new sets of data were measured quasi - simultaneously in both frequency bands during this thesis project. These data sets were measured with the $\mathrm{Tx}$ in different, nearby locations. One location was at site Txl which was the same as that used for the previous measurements. The other was at site $\mathrm{Tx} 2^{3}$ which was around the corner from site $\mathrm{Tx} 1$, on a street that ran in a north-south direction. This section reports propagation data analysis results for the three measurement sets separately.

\subsubsection{Results from analyses of the old set of data}

Measurement data from 27 different street sections in downtown Ottawa were analysed to obtain results reported in this section. Although many more measurements were made, sorting of the data revealed that there were only 27 cases in which it could be stated that 2 and $6 \mathrm{GHz}$ measurements were made in approximately the same locations. A map of the measurement area showing these street sections can be seen in Fig.3.2.10.

\footnotetext{
${ }^{2}$ Site Txl was on Laurier Avenue, between Kent St. and Bank St.

${ }^{3}$ Site Tx2 was on Kent Street, between Gloucester St. and Laurier Ave.
} 


\subsection{Relative powers of the three strongest MPGs}

For each street section (450 SREs in a 1-sec-long time series), the relative powers for the three strongest MPGs were estimated from the APDPs. The medians of the relative powers for the three strongest MPGs are listed in Table 3.1.

Table 3.1: Medians of the relative powers for the three strongest MPGs from 27 street sections:

\begin{tabular}{|l|l|l|l|}
\hline \multirow{2}{*}{ Frequency Band } & \multicolumn{3}{|l|}{ Median of relative average power (dB) } \\
\cline { 2 - 4 } & $1^{\text {st }}$ MPG & $2^{\text {nd }}$ MPG & $3^{\text {rd }}$ MPG \\
\hline $2 \mathrm{GHz}$ & 0 & -5 & -11 \\
\hline $6 \mathrm{GHz}$ & 0 & -7 & -10 \\
\hline
\end{tabular}

From Table 3.1, one can observe that the $3^{\text {rd }}$ MPG already has very low power, so that having more than 3 active fingers in a Rake receiver is probably not very beneficial as reported in [3]. 


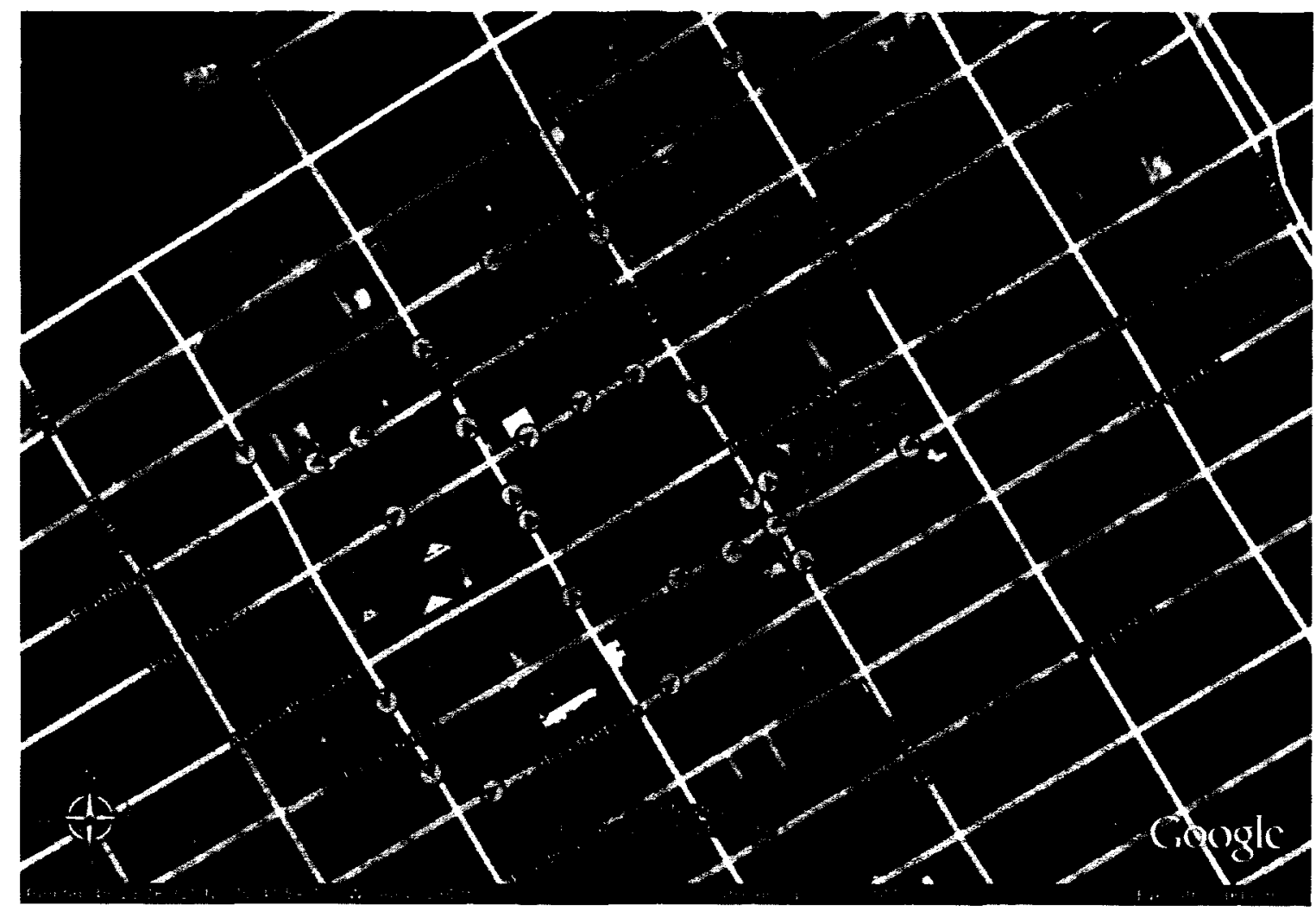

Fig.3.2 10: Map showing measurement locations for the old set of data, recorded with Tx on Laurier Avenue, between Kent St. and Bank St. 


\subsection{Fading statistics}

The envelope fading distributions for the three strongest MPGs on each street section were analysed. The ECDFs of fading ranges for the old set of data are shown in Fig.3.2.11.

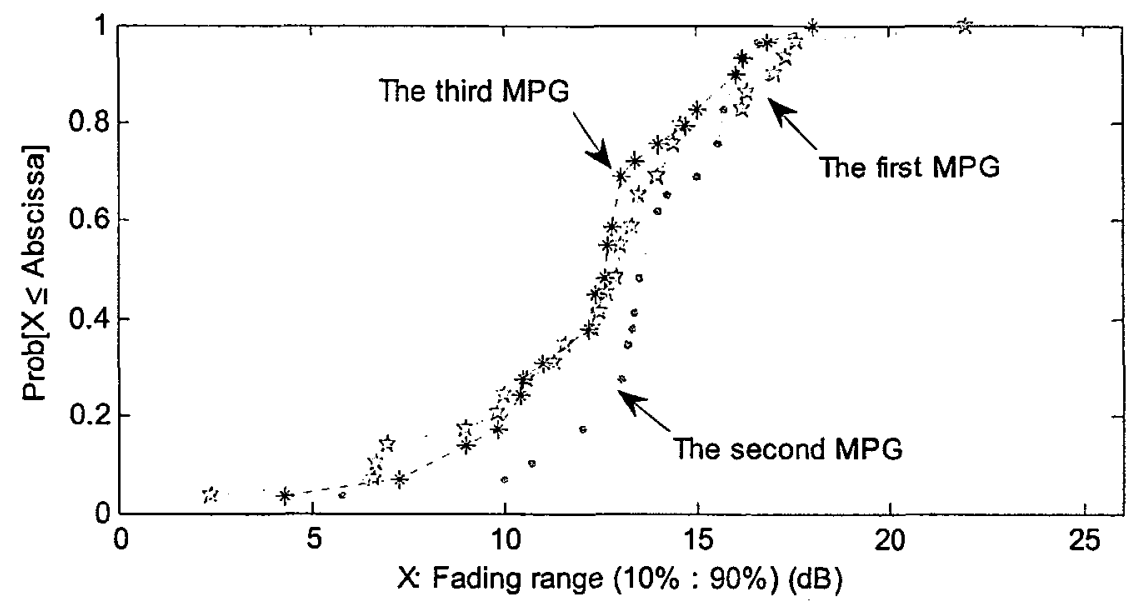

(a) ECDFs of fading ranges for MPGs at $2 \mathrm{GHz}$

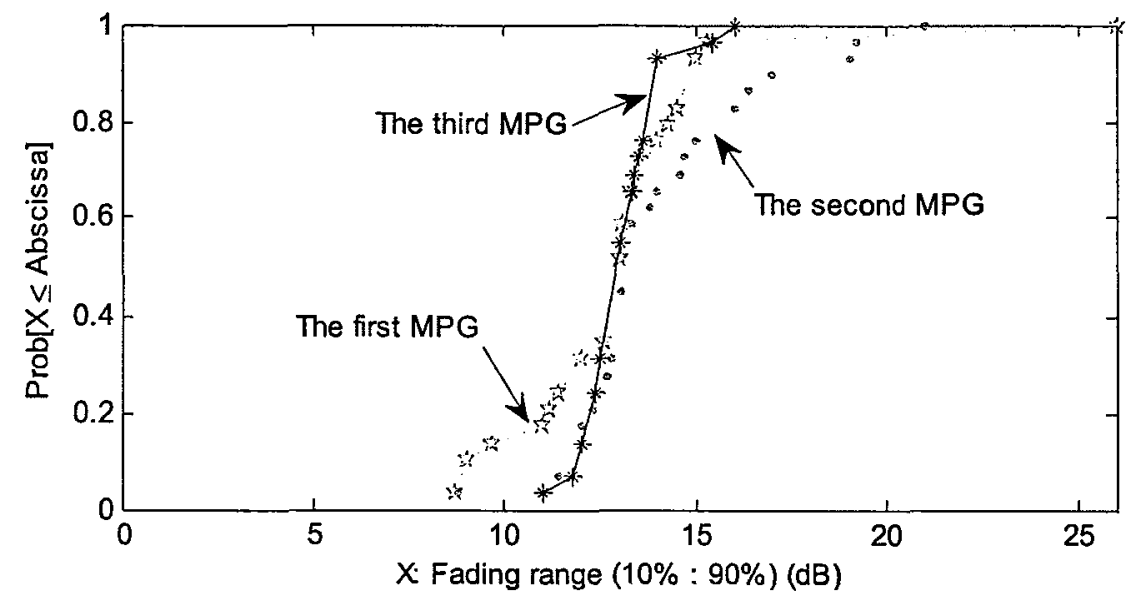

(b) ECDFs of fading ranges for MPGs at $6 \mathrm{GHz}$

Fig.3.2.11: ECDFs of fading ranges for the three strongest MPGs from 27 street sections in downtown Ottawa. 
The above figure shows that the changes of fading ranges for the MPGs are slightly wider at $2 \mathrm{GHz}$ than that at $6 \mathrm{GHz}$, but the median results that are listed in Table 3.2 for both frequencies show that the average fading behaviour is almost the same at 2 and 6 GHz. Firm conclusions can't be drawn because the data pool is too small.

Table 3.2: Medians of envelope fading ranges (10\%: 90\%) for 27 street sections.

\begin{tabular}{|l|l|l|l|}
\hline \multirow{2}{*}{ Frequency Band } & \multicolumn{3}{|l|}{ Median of envelope fading ranges (10\%: 90\%) (dB) } \\
\cline { 2 - 4 } & $1^{\text {st }}$ MPG & $2^{\text {nd }}$ MPG & $3^{\text {rd }}$ MPG \\
\hline $2 \mathrm{GHz}$ & 13 & 14 & 13 \\
\hline $6 \mathrm{GHz}$ & 13 & 13 & 13 \\
\hline
\end{tabular}

The fading statistics were studied for the old set of data, after Rake combining of MPGs with powers relative to that of the strongest MPG that were greater than $-7 \mathrm{~dB}$, as shown in Fig.3.2.12. Figure (a) shows ECDFs of fading ranges after Rake combining at 2 and $6 \mathrm{GHz}$. The median of fading ranges at $2 \mathrm{GHz}$ is $9.2 \mathrm{~dB}$, and the median of fading ranges at $6 \mathrm{GHz}$ is $10.1 \mathrm{~dB}$. The small difference between these results is not considered to be significant given the small number of street sections analysed. Figure (b) shows a scatter plot of the above fading ranges. One can see that the fading ranges at $2 \mathrm{GHz}$ were slightly greater on about $30 \%$ of the street sections and less on about $55 \%$ of the street sections. The fading range was the same at both frequencies on about $15 \%$ of the street sections. This indicates that after Rake combining, at $6 \mathrm{GHz}$, the decision variable would suffer a wider range of fading than that at $2 \mathrm{GHz}$ data in the majority of street sections. This is expected because the wavelength is shorter at $6 \mathrm{GHz}$, so the phases of received multipath components vary more rapidly in space and time, causing a higher probability of both fades and enhancements. 


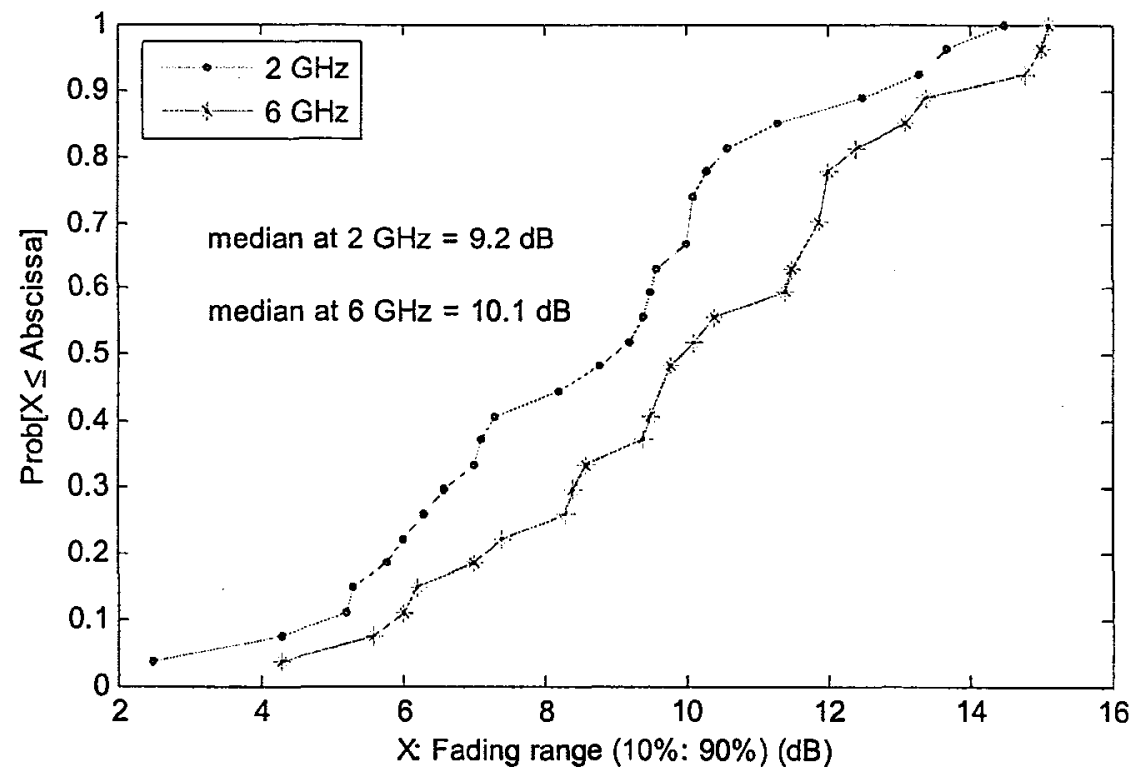

(a) ECDFs of fading ranges after Rake combining of MPGs

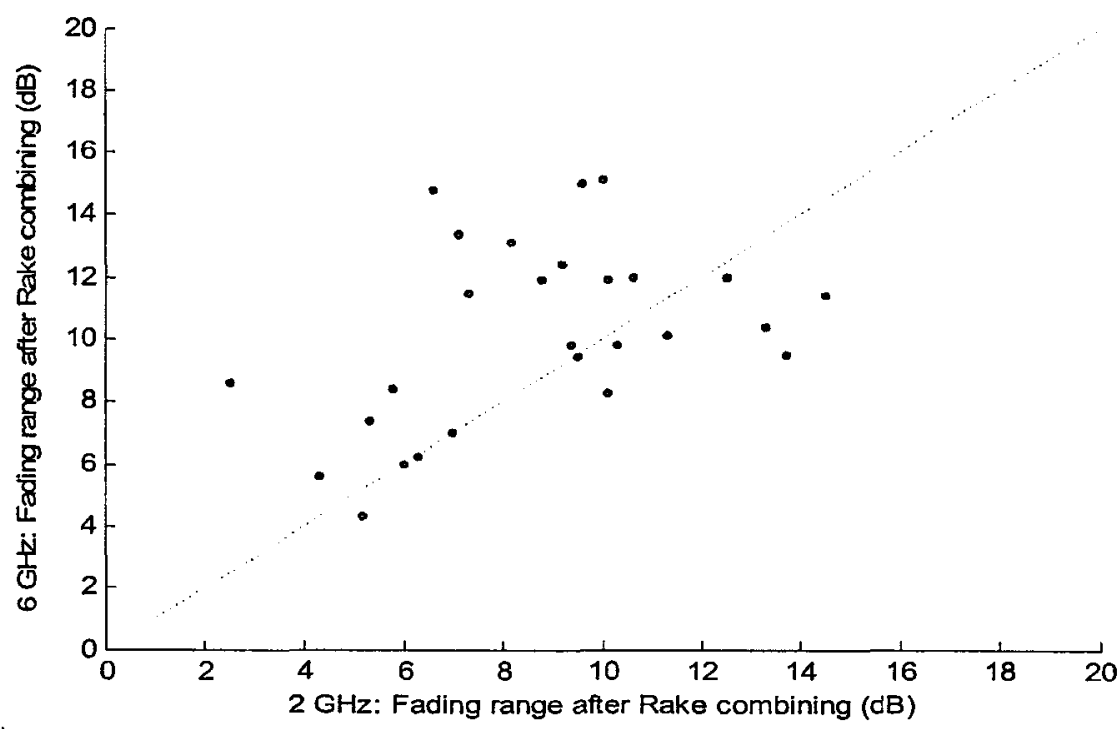

(b) Scatter plot of envelope fading ranges (10\%: $90 \%)$ after Rake combining

Fig.3.2.12: ECDFs and scatter plot of fading ranges after Rake combining for the MPGs with relative power greater than $-7 \mathrm{~dB}$ for the old set of data at 2 and $6 \mathrm{GHz}$ with $\mathrm{Tx}$ at site $\mathrm{Tx} 1$. 
Since the measured data were recorded over the same 1-sec-long interval in both frequency bands, there is a higher probability that phase relationships that give rise to deep fades occur at $6 \mathrm{GHz}$ than that at $2 \mathrm{GHz}$ within $1-\mathrm{sec}$ interval. It is likely, however, that if the data recording for the $2 \mathrm{GHz}$ band was made longer, such CDFs would show that fading to the same depths was equally probable in both bands.

\subsection{Correlation coefficient of fading envelopes for MPGs}

Cross correlations between the fading of the envelopes of different MPGs were studied. The correlation coefficients for the three strongest MPGs in each channel were estimated. The average correlation coefficient matrices for the old set of data are shown as:

$$
\begin{aligned}
& \left\{\begin{array}{lll}
1.0000 & 0.0084 & 0.0367 \\
0.0084 & 1.0000 & 0.0457 \\
0.0367 & 0.0457 & 1.0000
\end{array}\right\} \text { at } 2 \mathrm{GHz} \\
& \left\{\begin{array}{lll}
1.0000 & 0.0002 & 0.0029 \\
0.0002 & 1.0000 & 0.0231 \\
0.0029 & 0.0231 & 1.0000
\end{array}\right\} \text { at } 6 \mathrm{GHz}
\end{aligned}
$$

The result indicates MPGs have lower correlations at $6 \mathrm{GHz}$ than at $2 \mathrm{GHz}$, and the correlation of selected MPGs in both bands is sufficiently small as to indicate that Rake combining might lead to better BER performance. 


\subsection{RMS delay spread}

The RMS delay spreads were estimated. Fig.3.2.13 shows ECDFs of RMS delay spreads in both bands in NLOS cases. The figure indicates slightly greater values at $2 \mathrm{GHz}$ with the median of $313 \mathrm{~ns}$, compared to that of $288 \mathrm{~ns}$ at $6 \mathrm{GHz}$ for the old set of data when the $T x$ was at $T x 1$.

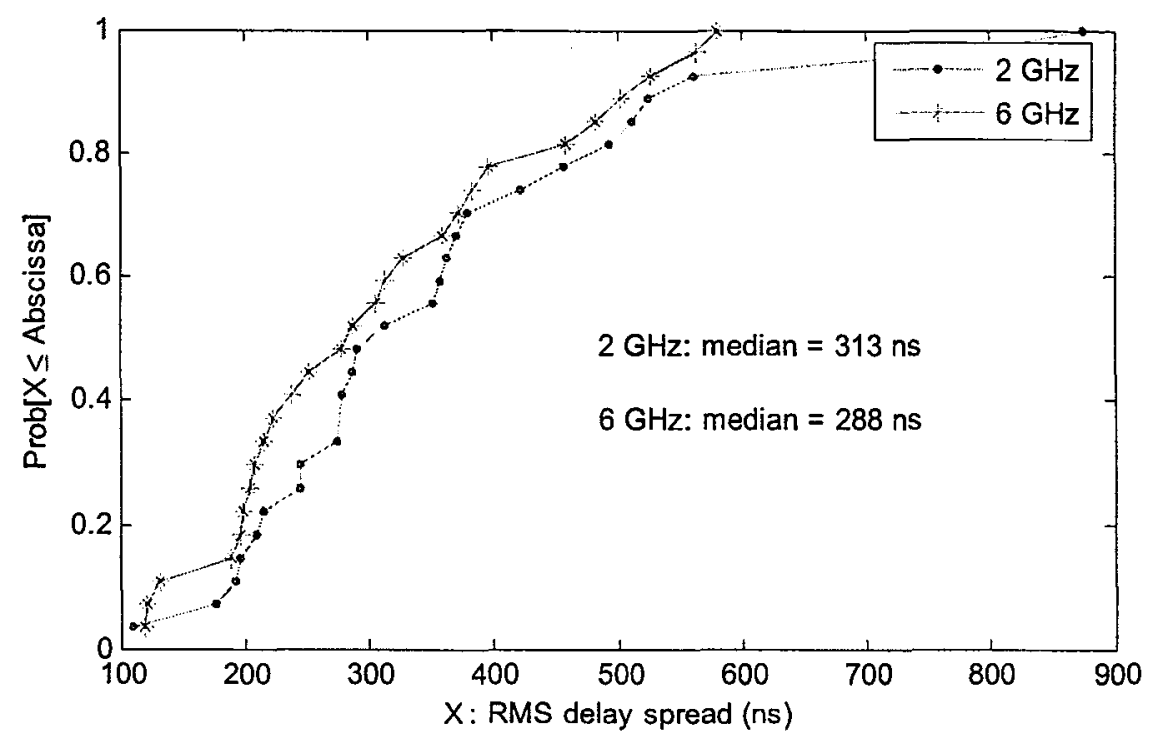

Fig.3.2.13: ECDFs of RMS delay spreads for the old set of data at 2 and $6 \mathrm{GHz}$ in downtown Ottawa. 


\subsubsection{Results from analyses of the new set of data ( $\mathrm{T} x$ on Kent Street, between Gloucester St. and Laurier Ave.)}

The analyses of data from 48 street sections, measured when the Tx was at site Tx2, are presented in this section. A map showing the measurement locations can be seen in Fig.3.2.14. Both LOS and NLOS cases are considered.

\subsection{Relative powers of the first three strongest MPGs}

The relative powers for the three strongest MPGs were estimated and analysed. The medians are listed in Table 3.3.

Table 3.3: Medians of relative average powers for the three strongest MPGs from 48 street sections:

\begin{tabular}{|l|l|l|l|}
\hline \multirow{2}{*}{ Frequency Band } & \multicolumn{3}{|l|}{ Median of relative average power (dB) } \\
\cline { 2 - 4 } & $1^{\text {st }}$ MPG & $2^{\text {nd }} M P G$ & $3^{\text {rd }} M P G$ \\
\hline $2 \mathrm{GHz}$ & 0 & -12 & -16 \\
\hline $6 \mathrm{GHz}$ & 0 & -12 & -16 \\
\hline
\end{tabular}

Table 3.3 shows that the relative powers of the second and third MPGs for this set of data measured with the $\mathrm{Tx}$ at site $\mathrm{Tx} 2$ are much lower compared with those of the old set of data, measured with the Tx at site Tx1, which is on Laurier Avenue, between Kent St. and Bank St. It is believed that this is because there is more open space close to Tx 2 than there is in the immediate proximity of $\mathrm{Tx} 1$. 


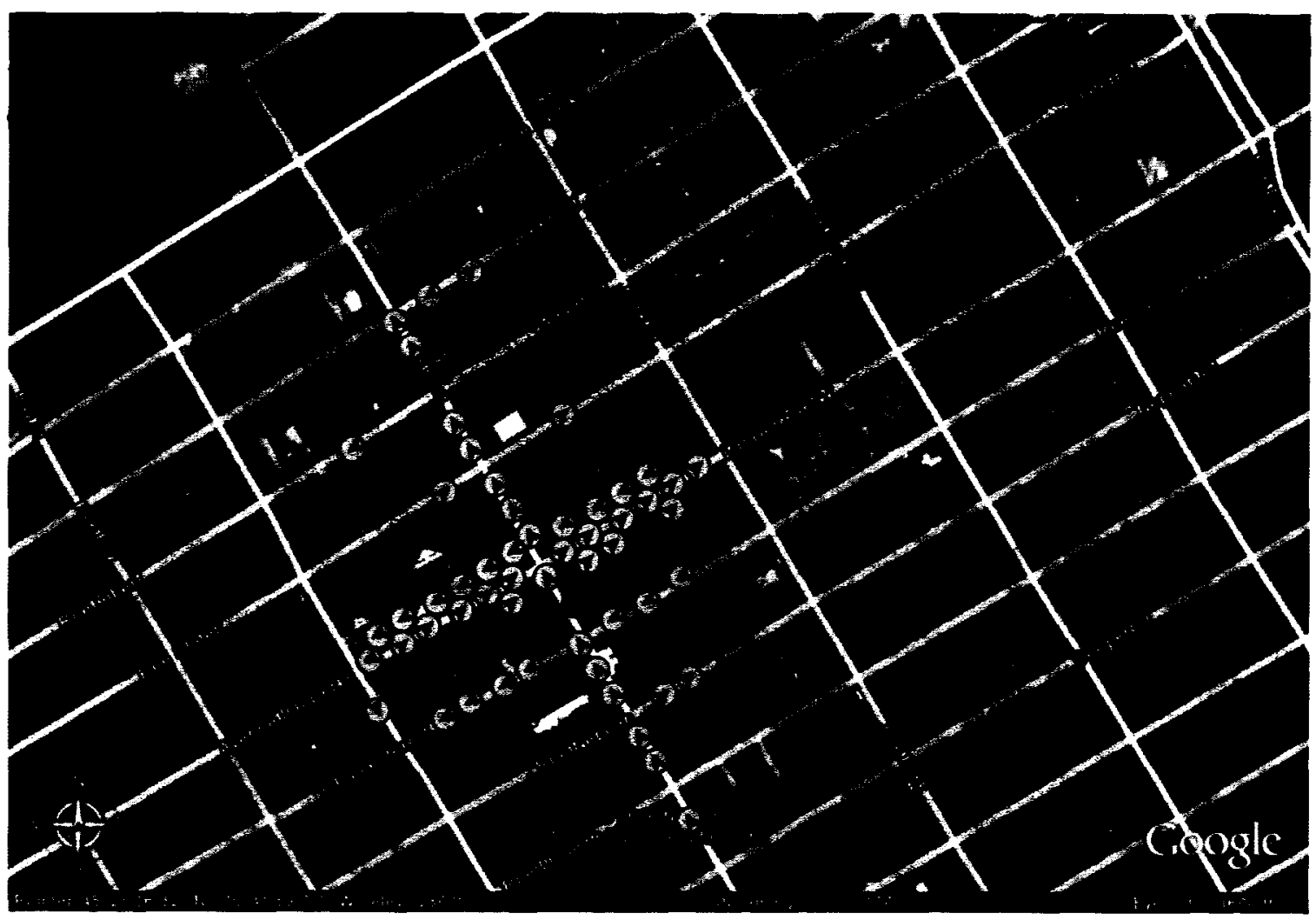

Fig.3.2.14: Map showing measurement locations for the new set of data, recorded with the Tx on Kent Street, between Gloucester St. and Laurier Ave. 


\subsection{Fading statistics}

The envelope fading distributions for the three strongest MPGs on each street section were analysed. The ECDFs of fading ranges for the set of data at 2 and $6 \mathrm{GHz}$ are shown in Fig.3.2.15.

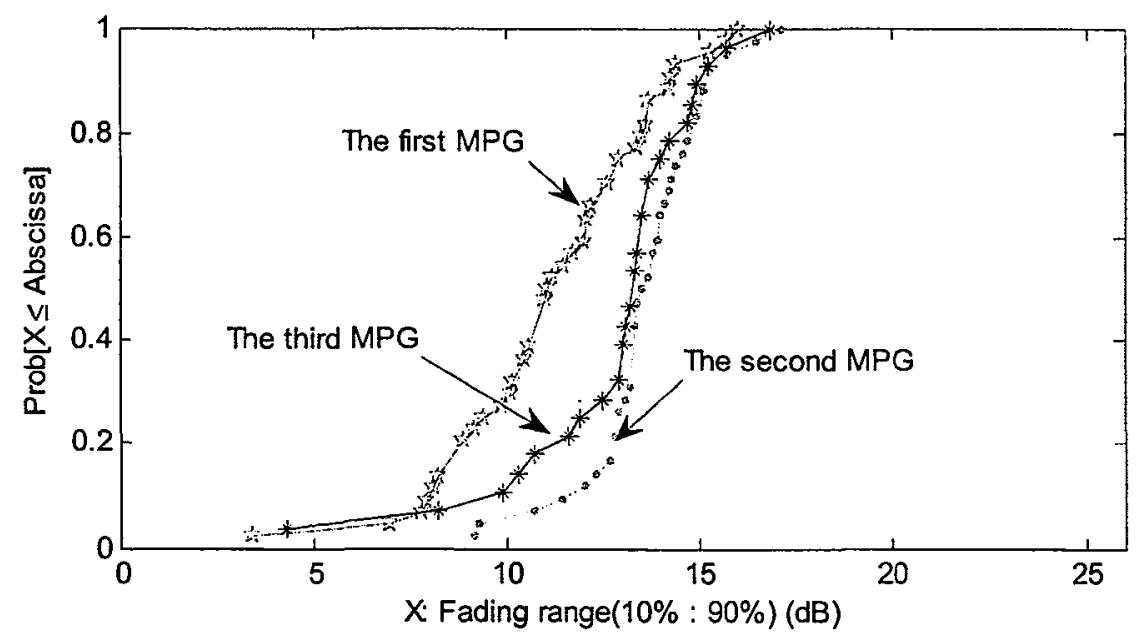

(a) ECDFs of fading ranges for MPGs at $2 \mathrm{GHz}$

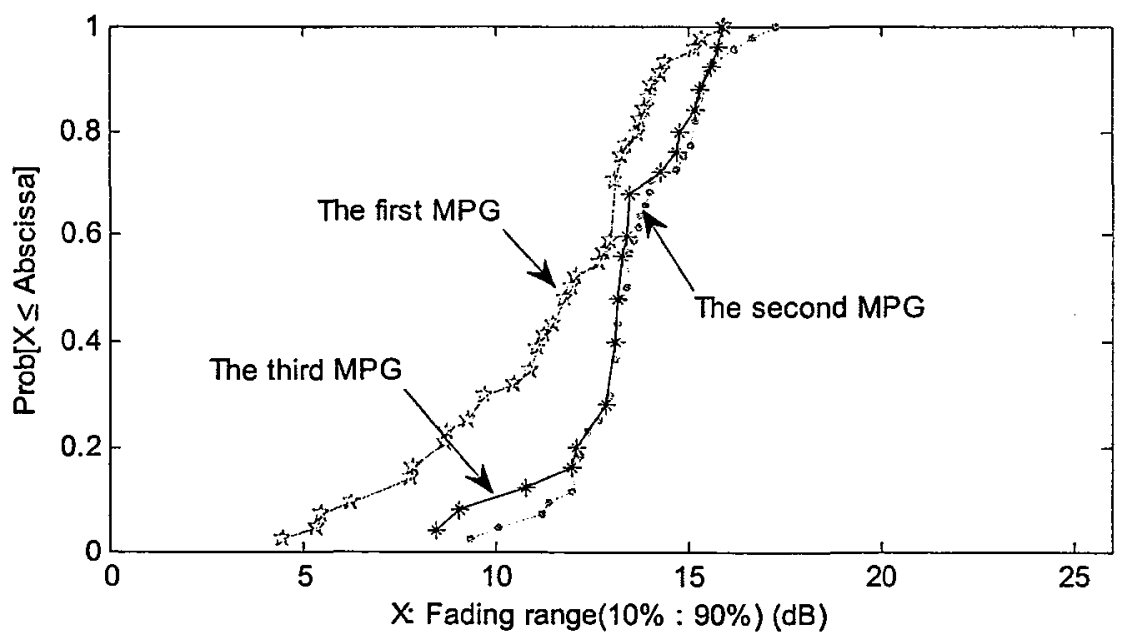

(b) ECDF of fading ranges for MPGs at $6 \mathrm{GHz}$

Fig.3.2.15: ECDFs of fading ranges for the three strongest MPGs from 48 street sections in downtown Ottawa. 
Figure 3.2.15 shows that the changes of fading ranges for the MPGs are almost same at both frequencies. Table 3.4 lists the median results, showing that the average fading behaviours are almost the same at 2 and $6 \mathrm{GHz}$ for the set of measurement. Again, firm conclusions can't be drawn about the comparison between the 2 and $6 \mathrm{GHz}$ results because the data pool is too small, as there should be more than 100 independent samples from reasonable statistical significance [23].

Table 3.4: Medians of envelope fading ranges (10\%: 90\%) for 48 street sections.

\begin{tabular}{|l|l|l|l|}
\hline \multirow{2}{*}{ Frequency Band } & \multicolumn{3}{|l|}{ Median of envelope fading ranges (10\%: 90\%) (dB) } \\
\cline { 2 - 4 } & $1^{\text {st }}$ MPG & $2^{\text {nd }}$ MPG & $3^{\text {rd }}$ MPG \\
\hline $2 \mathrm{GHz}$ & 11 & 14 & 13 \\
\hline $6 \mathrm{GHz}$ & 12 & 14 & 13 \\
\hline
\end{tabular}

The fading statistics were studied for this set of data, after Rake combining of MPGs with powers relative to that of the strongest MPG that were greater than $-7 \mathrm{~dB}$, as shown in Fig.3.2.16. Figure (a) shows the ECDFs of fading ranges for MPGs after Rake combining. The median of fading ranges at $2 \mathrm{GHz}$ is $11 \mathrm{~dB}$, and the median of fading ranges at $6 \mathrm{GHz}$ is $11.9 \mathrm{~dB}$. Figure (b) shows a scatter plot of the above fading ranges. one can get the fading ranges in the two frequency bands were equal on about $5 \%$ of the street sections, greater at $2 \mathrm{GHz}$ on about $45 \%$, and greater at $6 \mathrm{GHz}$ on about $50 \%$ of measured street sections. 


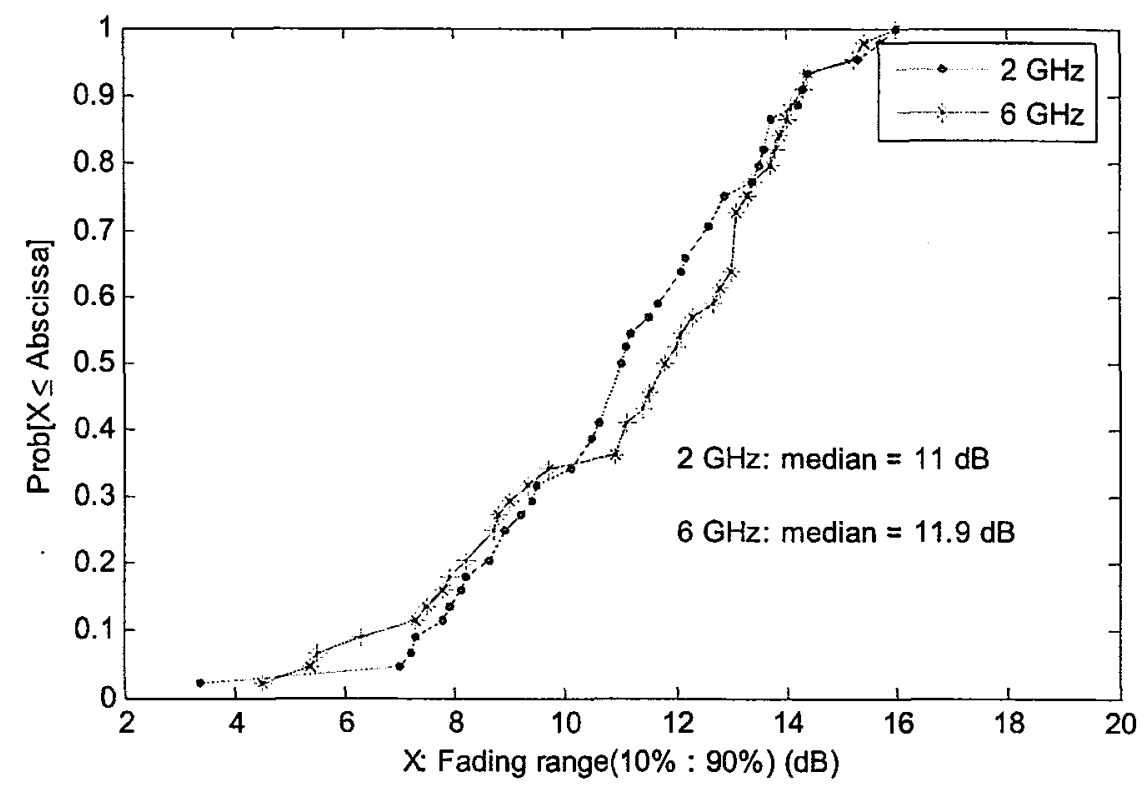

(a) ECDF of fading ranges after Rake combining of MPGs

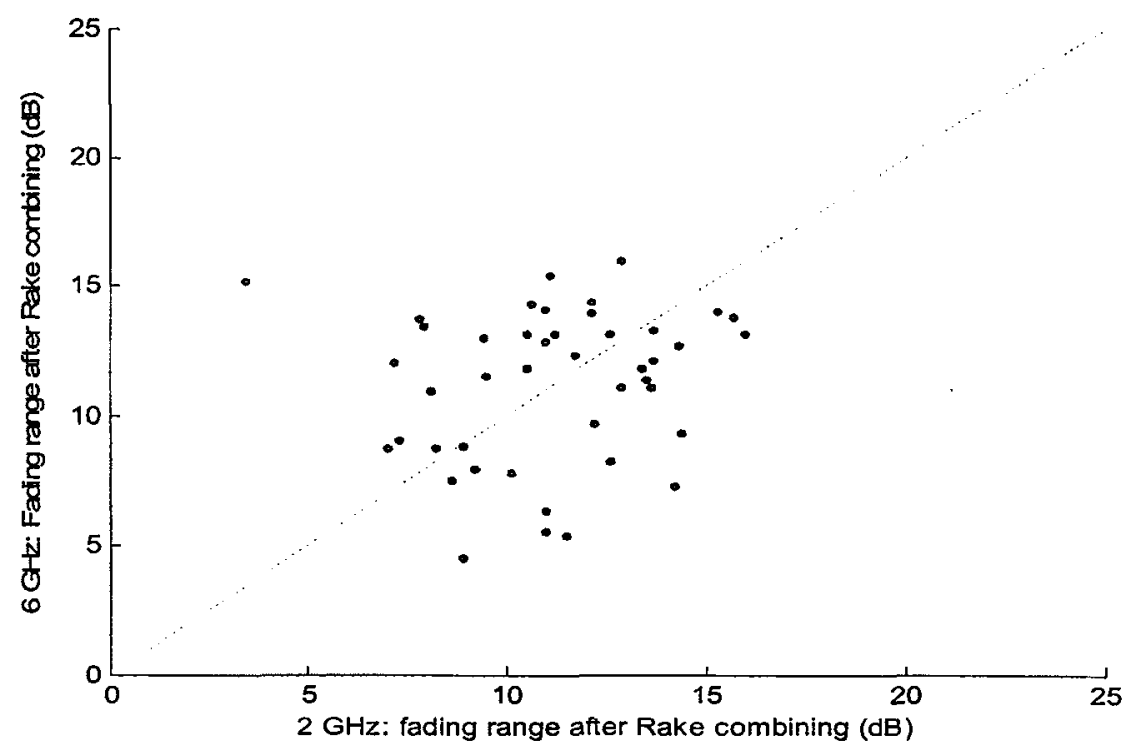

(b) Scatter plot of envelope fading ranges (10\%: $90 \%$ ) after Rake combining

Fig.3.2.16: ECDFs and scatter plot of fading ranges after Rake combining for the MPGs with relative power greater than $-7 \mathrm{~dB}$ for 48 street sections with $\mathrm{Tx}$ at site $\mathrm{Tx} 2$. 


\subsection{RMS delay spread}

The RMS delay spreads for both LOS and NLOS street sections were calculated and the ECDFs of the RMS delay spreads for the set of data at 2 and $6 \mathrm{GHz}$ are shown in Fig.3.2.17. The results show that the median of RMS delay spreads at both frequencies are almost same in LOS, and slightly greater at $2 \mathrm{GHz}$ with the median of $200 \mathrm{~ns}$ compared with the median of $160 \mathrm{~ns}$ at $6 \mathrm{GHz}$ in NLOS.

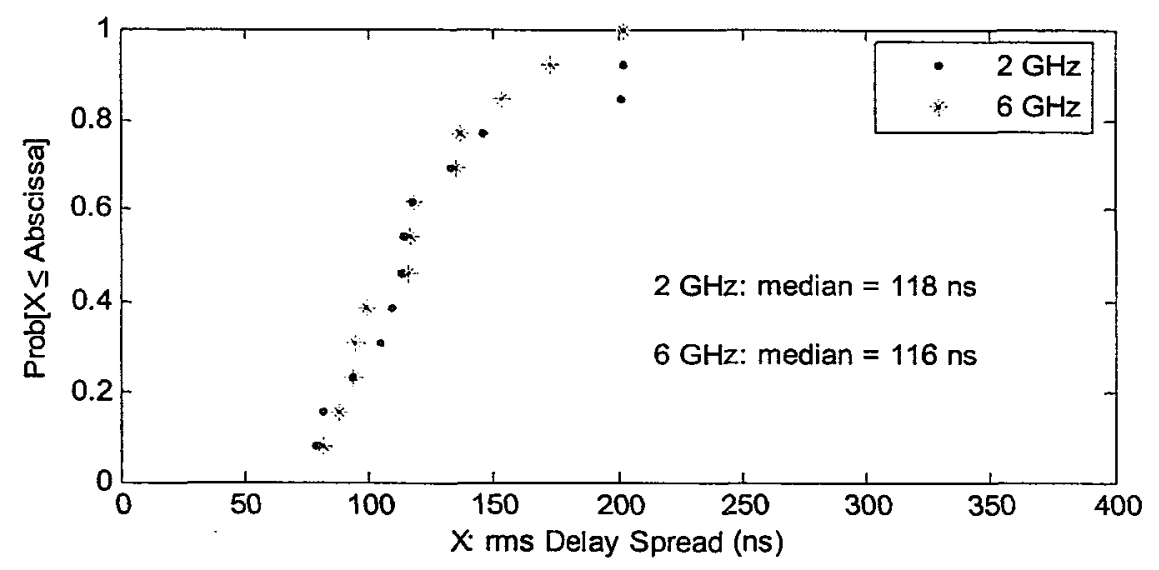

(a) RMS delay spreads for 14 LOS street locations.

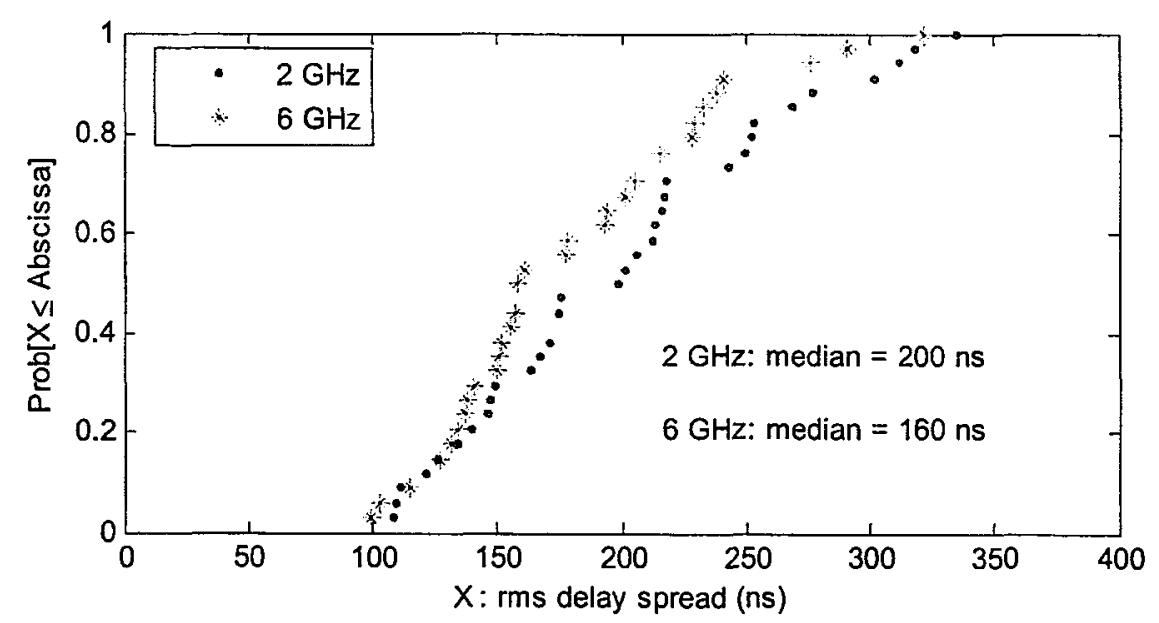

(b) RMS delay spreads for 34 NLOS street locations.

Fig.3.2.17: ECDFs of RMS delay spread for 48 street sections with $\mathrm{Tx}$ at site $\mathrm{Tx}$. 


\subsubsection{Results from analyses of the new set of data $(\mathrm{Tx}$ on Laurier Avenue, between Kent St. and Bank St.)}

Results of propagation data analyses for 147 street sections, measured when the Tx was at site $T x 1$, are presented. A map showing the measurement locations can be seen in Fig.3.2.18. The analyses include LOS and NLOS cases.

\subsection{Relative powers of the first three strongest MPGs}

The relative powers for the three strongest MPGs were estimated and analysed for each of the radio channel. The medians are listed in Table 3.5.

Table 3.5: Medians of relative average powers for the three strongest MPGs from 147 street sections:

\begin{tabular}{|l|l|l|l|}
\hline \multirow{2}{*}{ Frequency Band } & \multicolumn{3}{|l|}{ Median of relative average powers (dB) } \\
\cline { 2 - 4 } & $1^{\text {st }}$ MPG & $2^{\text {nd }}$ MPG & $3^{\text {rd }}$ MPG \\
\hline $2 \mathrm{GHz}$ & 0 & -6 & -10 \\
\hline $6 \mathrm{GHz}$ & 0 & -7 & -11 \\
\hline
\end{tabular}

Comparison of Table 3.1 and Table 3.5 shows that the relative powers of the second and third MPGs for the two sets of data are almost same. The reason is that the two sets of data were recorded with the Tx on the same site $-\mathrm{Tx} 1$. Table 3.3 shows the relative powers of the second and third MPGs evaluated from the data recorded with the Tx at Tx2 are low compared with those in Table 3.1 and Table 3.5. This indicates that considerable variation in channel parameters can be expected for different microcell base station when the density and height of buildings is non-uniform, as in downtown Ottawa. 


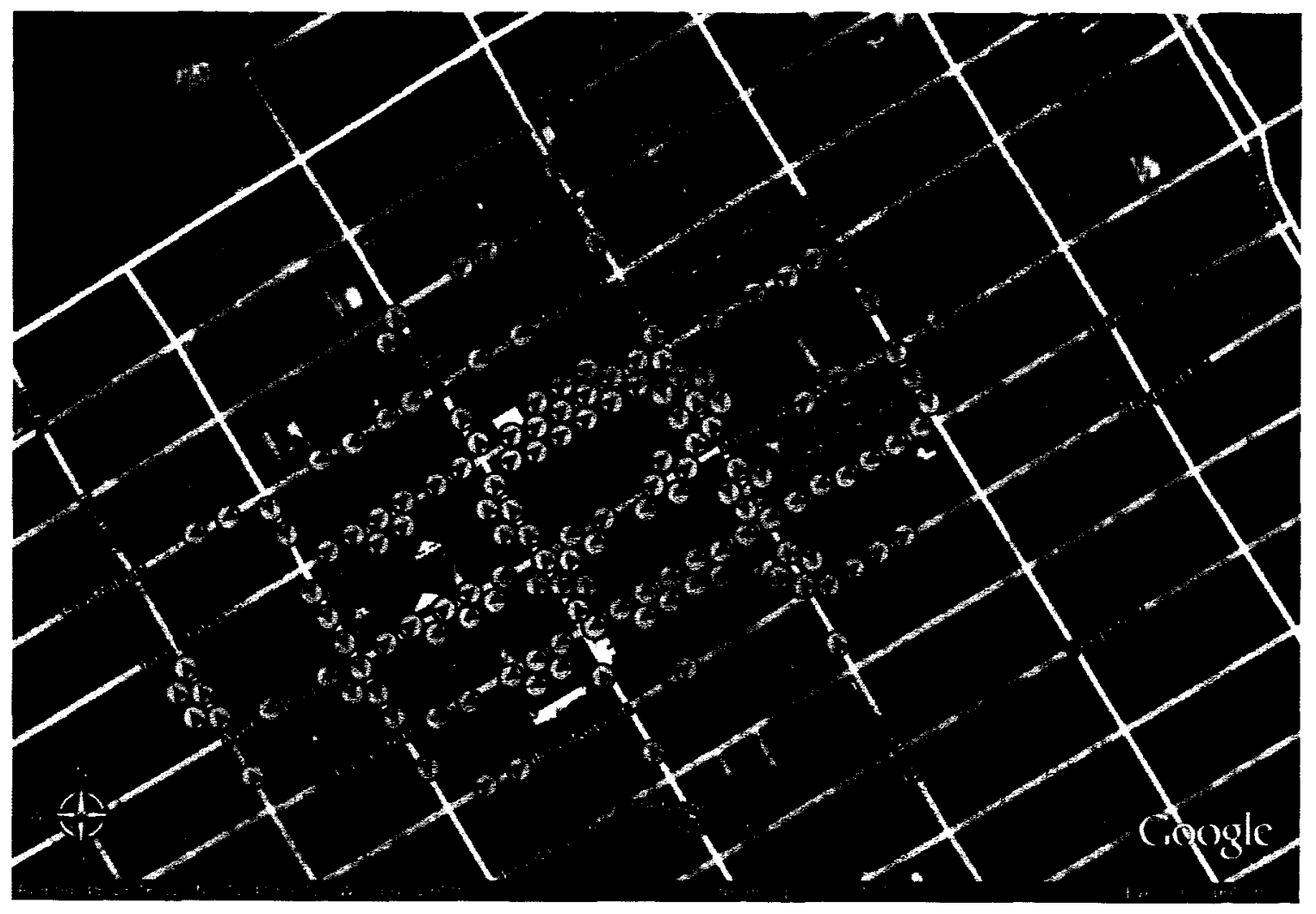

Fig.3.2.18: Map showing measurement locations for the new set of data, recorded with the Tx on Laurier Avenue, between Kent St. and Bank St. 


\subsection{Fading statistics}

The envelope fading distributions for the three strongest MPGs on each street section were analysed. ECDFs of fading ranges for the three strongest MPGs are shown in Fig.3.2.19.

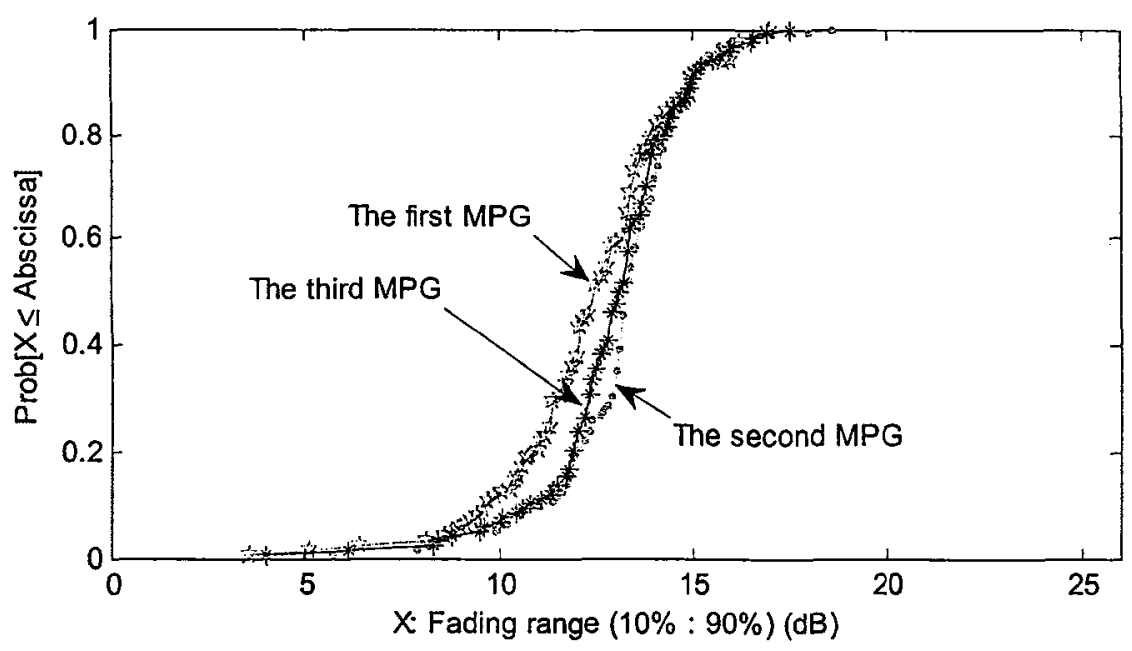

(a) ECDFs of fading ranges for MPGs at $2 \mathrm{GHz}$

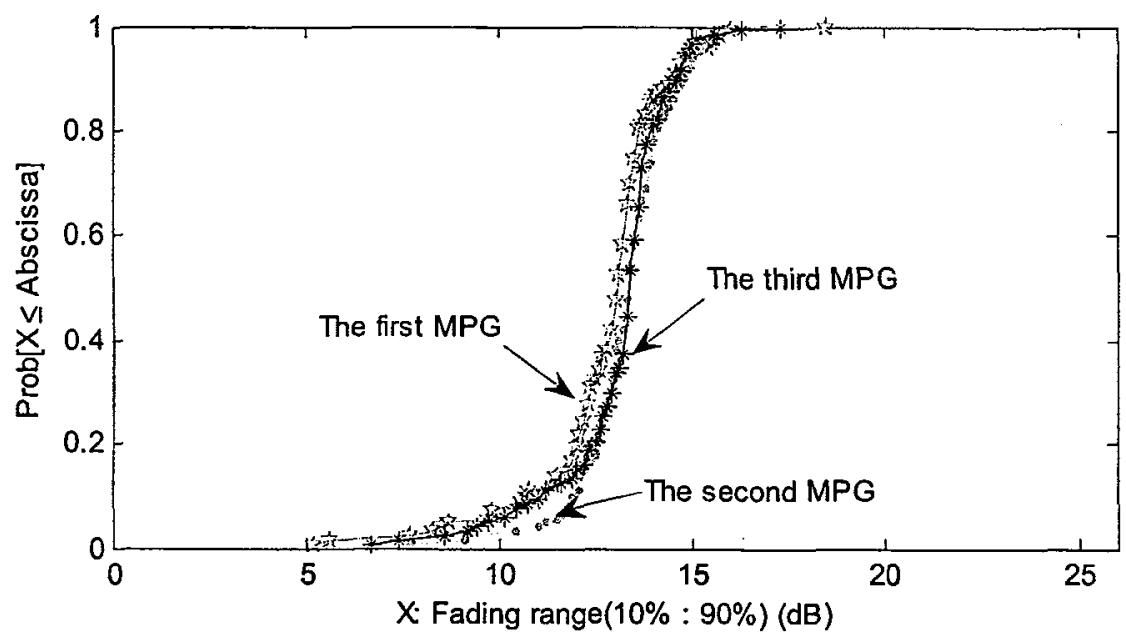

(b) ECDF of fading ranges for MPGs at $6 \mathrm{GHz}$

Fig.3.2.19: ECDFs of fading ranges for the three strongest MPGs from 147 street sections in downtown Ottawa. 
Figure 3.2.19 shows that the changes of fading ranges for the MPGs are almost the same at both frequencies. Table 3.6 lists the medians of envelope fading ranges (10\%: $90 \%$ ) for the above different cases, showing that the average fading behaviour is the same at 2 and $6 \mathrm{GHz}$.

Table 3.6: Medians of envelope fading ranges (10\%: 90\%) for 147 street sections.

\begin{tabular}{|l|l|l|l|}
\hline \multirow{2}{*}{ Frequency Band } & \multicolumn{3}{|l|}{ Median of envelope fading ranges (10\%: 90\%) (dB) } \\
\cline { 2 - 4 } & $1^{\text {st }} \mathrm{MPG}$ & $2^{\text {nd }} \mathrm{MPG}$ & $3^{\text {rd }} \mathrm{MPG}$ \\
\hline $2 \mathrm{GHz}$ & 13 & 13 & 13 \\
\hline $6 \mathrm{GHz}$ & 13 & 13 & 13 \\
\hline
\end{tabular}

The fading statistics were studied for this set of data, after Rake combining of MPGs with powers relative to that of the strongest MPG that were greater than $-7 \mathrm{~dB}$, as shown in Fig.3.2.20. Figure (a) shows the ECDFs of fading ranges for MPGs after Rake combining. The median of fading ranges at $2 \mathrm{GHz}$ is $9.1 \mathrm{~dB}$, and the median of fading ranges at $6 \mathrm{GHz}$ is $10.6 \mathrm{~dB}$. Figure (b) shows a scatter plot of the above fading ranges. From the figure, it can be estimated that the fading ranges at both frequency bands were equal on about $6 \%$ of the measured street sections, the fading ranges at $2 \mathrm{GHz}$ were slightly greater in about $30 \%$, and the fading ranges at $6 \mathrm{GHz}$ were greater in about $64 \%$. As previously stated, it can be concluded that, over the same time interval, there is a greater probability that, at $6 \mathrm{GHz}$, the output from a Rake combiner would be in a faded condition. 


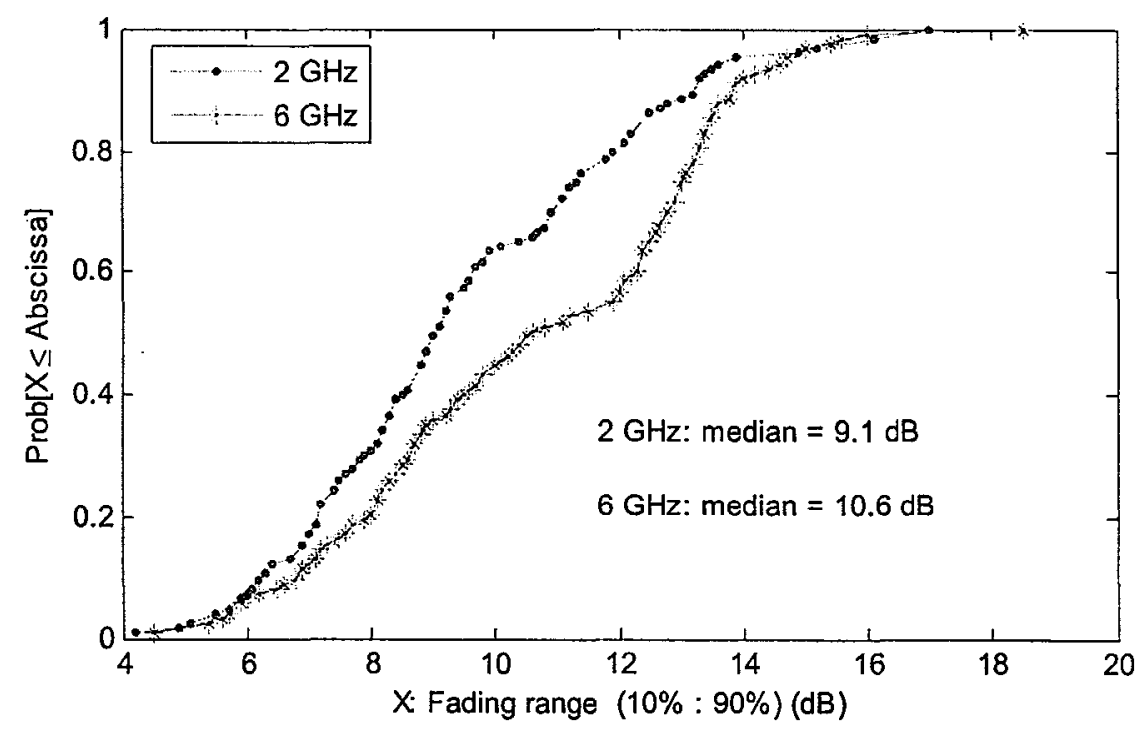

(a) ECDF of fading ranges after Rake combining of MPGs

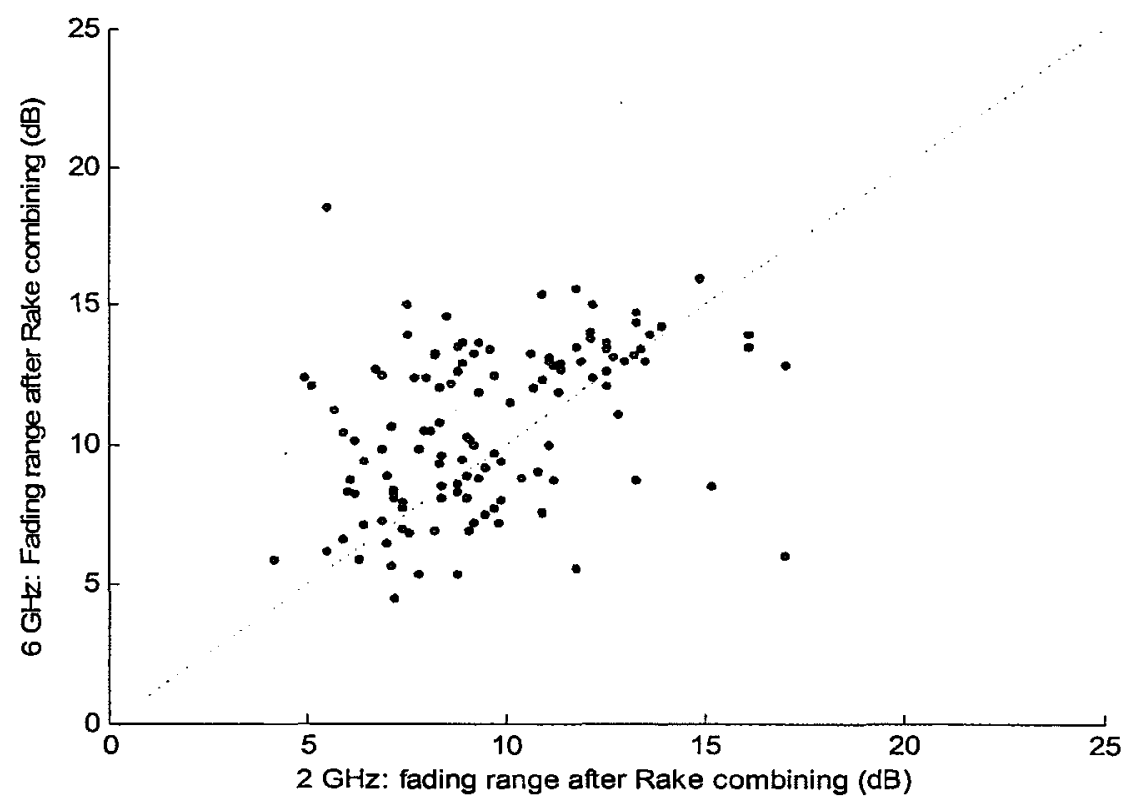

(b) Scatter plot of envelope fading ranges (10\%: 90\%) after Rake combining

Fig.3.2.20: ECDFs and scatter plot of fading ranges after Rake combining for the MPGs with relative power greater than $-7 \mathrm{~dB}$ for 147 street sections with $T x$ at site $T x 1$. 


\subsection{RMS delay spread}

The RMS delay spreads for LOS and NLOS were calculated and the ECDFs of the RMS delay spread at 2 and $6 \mathrm{GHz}$ are shown in Fig.3.2.21. The results show that $2 \mathrm{GHz}$ data have slightly greater RMS delay spread in both LOS and NLOS street locations.

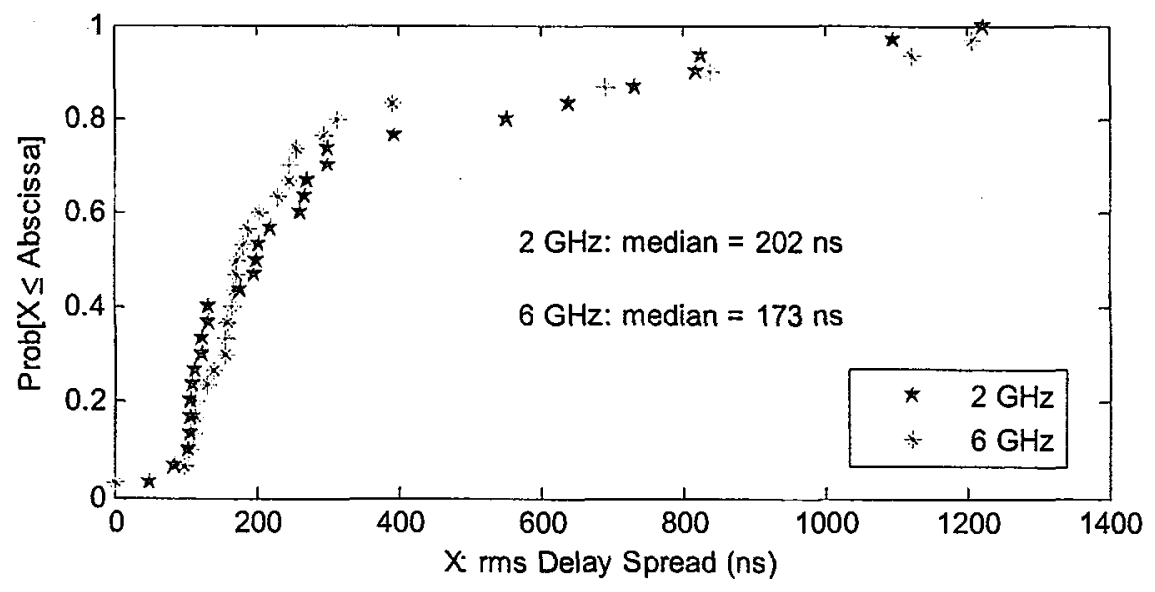

(a) RMS delay spreads for 30 LOS street locations.

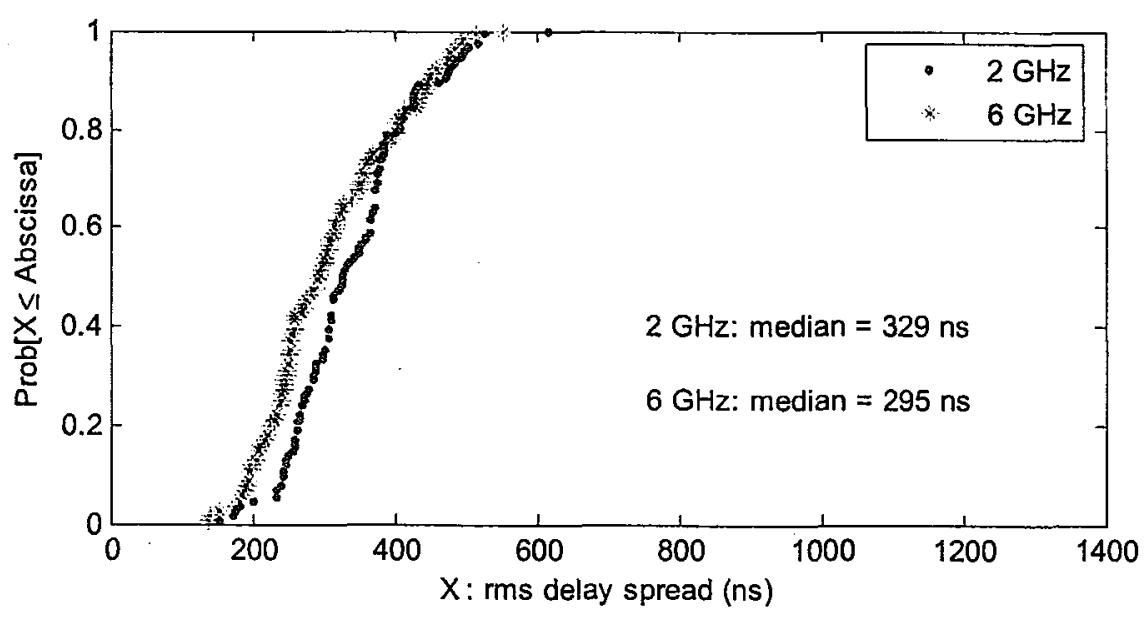

(b) RMS delay spreads for 117 NLOS street locations.

Fig.3.2.21: ECDFs of RMS delay spread for 147 street sections with Tx at site Tx1at 2 and $6 \mathrm{GHz}$. 


\subsection{Conclusions}

Based on the analyses in the previous sections, there are several conclusions:

A. Envelope fading for different MPGs with 300 ns spacing exhibits little cross correlation with the average cross-correlation coefficient being within the range \pm 0.05 . Significant combining gains should therefore be available with Rake combining, if multiple MPGs are available with a small spread in relative powers.

B. Analysis results show that typically up to three MPGs with powers within a $7 \mathrm{~dB}$ range relative to the strongest MPG, are available for Rake combining.

C. The fading distributions of MPGs vary significantly with the location of transmitter. This indicates that considerable variation in channel parameters can be expected for different microcell base station locations when the density and height of buildings is non-uniform, as in downtown Ottawa.

D. In LOS, the RMS delay spreads at $2 \mathrm{GHz}$ and $6 \mathrm{GHz}$ are approximately the same, whereas in NLOS, more than $80 \%$ of the RMS delay spreads at $2 \mathrm{GHz}$ are slightly greater than those at $6 \mathrm{GHz}$. Reasons for this are not clear.

E. In the 1-sec-long time series analysed during work for this thesis, ECDFs of fading ranges for MPGs after Rake combining showed the fading ranges to be greater at 6 GHz. This is due to fact that the wavelength is shorter at $6 \mathrm{GHz}$ and thus, within any time or space interval, phase relationships that result in a fade are more probable. 


\section{Chapter 4}

\section{Semi-Analytical Prediction of BER Performance on DSSS Mobile Radio Links}

A semi-analytical technique (SAT), for predicating the BER performance of DSSS Rake receivers was reported earlier by Adnani [3]. This technique was applied to measured propagation data for high data rate BER predictions reported in this thesis. The theoretical basis for this technique is therefore reviewed in this chapter. Then, BER prediction results are reported. Finally, a comparison of BER predictions based on quasisimultaneous channel soundings in the 2 and $6 \mathrm{GHz}$ frequency bands and an explanation of the results based on knowledge of propagation parameters are presented.

\subsection{BER Prediction method}

The SAT is a method through which numerical integration of upper and lower bounds on distributions for SI can be used to calculate BERs in cases when SI must be accounted for [3]. Its use in a time series mode allows the substitution of channel models with actual channel soundings to give more accurate results for particular scenarios.

Analytical expressions for BER in multipath fading environments are normally derived under the following assumptions: 
- The MPGs received at different Rake fingers are assumed to have equal average power [1];

- The fading of the received MPGs is assumed to be Rayleigh and i.i.d [1];

- The relative time-delay between received MPGs is assumed to be an integer multiple of the chip period [17 ] [18];

Obviously, the above assumptions are not true in general. Due to time dispersion in frequency selective fading environments, the delayed MPGs appear randomly, and create unpredictable SI. Inclusion of an expression for the PDF of SI in analytical equations makes the calculation of BER very complex and intractable.

However, based on the semi-analytical technique reported in the literature by Lehnert and Pursley to analyze multiple-access interference in non-fading environments, Adnani [3] made extensions to develop a new SAT for application to the problem of SI in multipath fading environments where DSSS radio links must sometimes operate.

Consider again equation (2.3) of Chapter 2 for the normalized decision variable at the output of a Rake receiver with L Rake fingers:

$$
D_{\text {Rake }, \text { norm }}=N_{d} b \sum_{i=1}^{L}\left|h_{i}\right|^{2}+\sum_{i=1}^{L} \sum_{k=1, k \neq i}^{K} h_{k, i} \frac{\cos \theta_{k, i}}{T_{c}} W_{k, i}+\frac{1}{T_{c}} \sqrt{\frac{T_{d}}{2 E_{b}}} \eta_{\text {Rake }}
$$

where the second term is the SI caused by multipath propagation, and $W_{k, i}=\int_{\alpha_{i}}^{\tau_{d}+\tau_{i}} b\left(t-\tau_{k}\right) a\left(t-\tau_{k}\right) a\left(t-\tau_{i}\right) d t$

is the cross-correlation between interfering binary sequence $k$ and the reference sequence i. The interference from the $k^{\text {th }}$ MPG to the $i^{\text {th }}$ MPG is assumed to be equal to the 
interference from the $i^{\text {th }}$ MPG to the $k^{\text {th }}$ MPG; also the phase term $\theta_{k, i}=\varphi_{k}-\varphi_{i}$ is assumed to be uniformly distributed on $[0.2 \pi]$.

For a (single) correlator receiver, (i.e. a receiver with $\mathrm{L}=1$ ), on a channel over which two MPGs are received, equation (4.1.1) can be written as:

$$
D_{1, n o r m}=N_{d} b\left|h_{1}\right|^{2}+h_{2,1} \frac{\cos \theta_{2,1}}{T_{c}} W_{2,1}+\frac{1}{T_{c}} \sqrt{\frac{T_{d}}{2 E_{b}}} \eta_{1}
$$

Thus, the SI term for the receiver with one Rake finger and two MPGs is:

$$
\begin{aligned}
& H_{2,1}=h_{2,1} \frac{\cos \theta_{2,1}}{T_{c}} W_{2,1}=\left|h_{1} \| h_{2}\right| I_{2,1} \cos \theta_{2,1} \\
& W_{2,1}=\int_{t_{1}}^{T_{1}+\tau_{1}} b\left(t-\tau_{2}\right) a\left(t-\tau_{2}\right) a\left(t-\tau_{1}\right) d t \\
& I_{2,1}=\frac{W_{2,1}}{T_{c}}
\end{aligned}
$$

A comprehensive analysis concerning the distribution of $I_{2,1}$ and a bounding technique to get the distribution bounds of $H_{2,1}$ (i.e. bounds on the distribution of SI) are reviewed in Appendix. The technique presents the expression for the upper and lower bounds of BER with consideration of the effects of SI.

\subsection{Verification of methodology}

This section shows the comparison of results from analytical BER predictions for DSSS BPSK, and BER predictions made using the SAT, for 1 and 2 tap Rayleigh fading channels. Fading of the two Rayleigh taps was simulated under an equal average power 
assumption and their fading was uncorrelated. Fig.4.2.1 shows the BER curves in different situations.

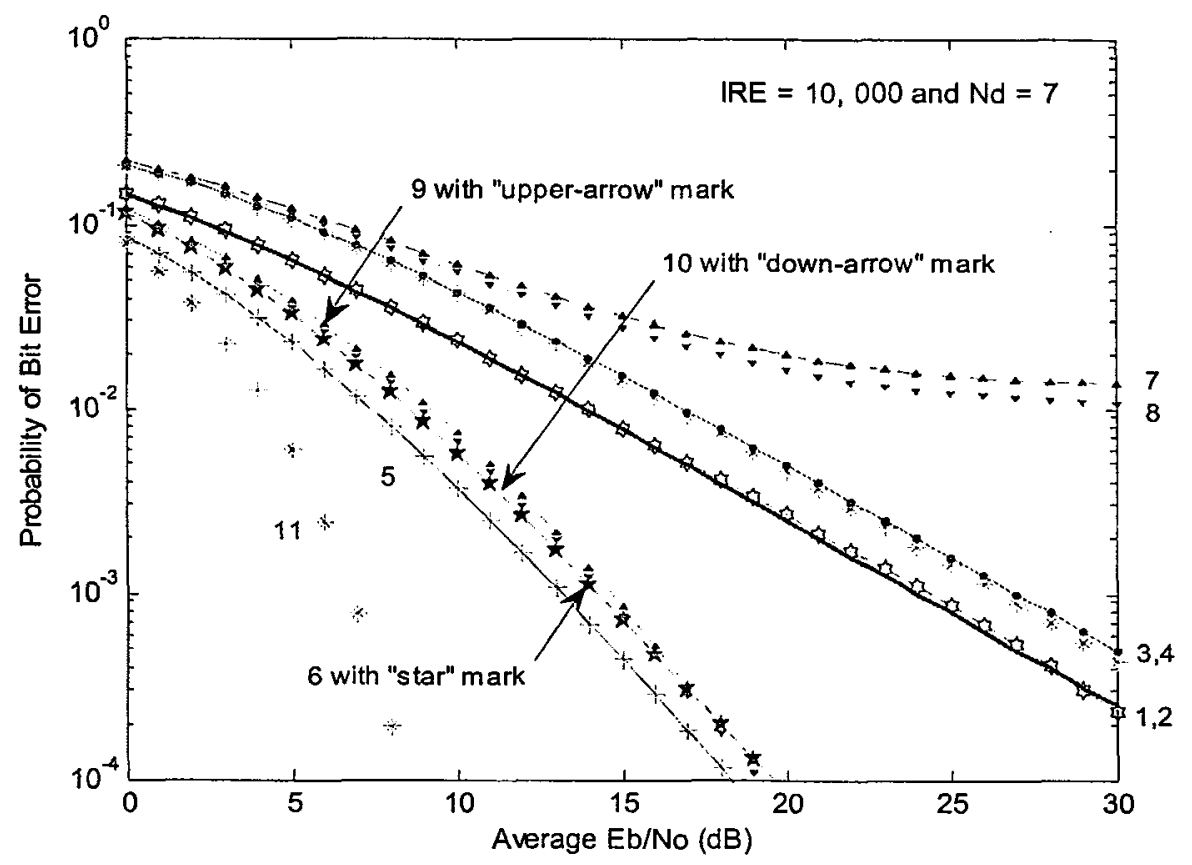

Fig.4.2.1: Comparison of analytical BER predictions with those made using the SAT for one-tap and two-tap Rayleigh fading channels.

In Fig.4.2.1, Curve 1, with the sold line shows the analytical BER result for a one-tap Rayleigh fading channel. The corresponding equation is well known and written as:

$\overline{\mathbf{P}}_{e}=\frac{1}{2}\left(1-\sqrt{\frac{\bar{\gamma}_{b}}{1+\bar{\gamma}_{b}}}\right)$

where $\vec{\gamma}_{b}=\frac{E_{b}}{N_{0}} * E\left(\alpha^{2}\right)$ the average signal-to-noise ratio and $E\left(\alpha^{2}\right)$ the average signal energy [1].

The BER for BPSK modulation on an additive white Gaussian noise (AWGN) channel is given by: 
$\mathrm{P}_{e}\left(\gamma_{b}\right)=Q\left(\sqrt{2 \gamma_{b}}\right)$

where $\gamma_{b}=\frac{E_{b}}{N_{0}}$ is the bit energy-to-noise power spectral density ratio.

Curve 2 with the open star markers shows the average BER result from application of the BER equation (4.2.2) for BPSK on a non-fading channel under the signal to noise ratio conditions appropriate to consecutive "instantaneous channel realizations" in the simulated Rayleigh fading time series.

For this case of 10,000 channel realizations, it can be seen that the analytical result from equation (4.2.1) and the time series average result are almost identical.

Based on the assumption that received MPGs have equal power, and exhibit Rayleigh i.i.d fading the analytical average BER for BPSK with $K$ received MPGs [1] is

$$
\overline{\mathrm{P}}_{e, M R C}=\left[\frac{1}{2}(1-\mu)\right]^{L} \sum_{\kappa=0}^{L-1}\left(\begin{array}{c}
L-1+\kappa \\
\kappa
\end{array}\right)\left[\frac{1}{2}(1+\mu)\right]^{\kappa}
$$

where $\mu=\sqrt{\frac{\bar{\gamma}_{c}}{1+\bar{\gamma}_{c}}}, \bar{\gamma}_{c}=\frac{E_{b}}{N_{0}} * E\left(\alpha_{\kappa}^{2}\right)$

Curve 3 with the solid dot markers shows the analytical BER if one-finger Rake receiver is used on the two-tap channel and no consideration is made of SI.

Curve 4 with the asterisk markers shows the time series average BER without consideration of SI in the case of two equal power MPGs and one Rake finger. It can be observed that curve 3 and curve 4 are very close. Comparison of these results with curve 1 and curve 2 shows that the required average $E_{b} / N_{o}$ for any specific error rate has increased by about $3 \mathrm{~dB}$ with respect to the case when the same power was received at a single Rake finger, since only half of the received energy is used by the Rx in this case. 
Curve 5 with the cross markers and Curve 6 with the closed star markers show the analytical BER results and those calculated via the time series averaging if a two-finger Rake receiver were used on the two-tap channel. In this case, all of the received energy was used for Rake combining. Comparison of these results shows that there is $1 \mathrm{~dB}$ discrepancy between curve 5 and curve 6 . The reason is the powers of two MPGs in simulation are not absolutely equal, resulting in less $1 \mathrm{~dB}$ lower combining gain compared with the well-know equation (4.2.3).

When the SAT, described previously, is properly applied to account for the effects of SI caused by the energy in the second MPG, the upper and lower bounds on BER for only one Rake finger are shown on curve 7 with up-arrow markers and curve 8 with downarrow markers, respectively. It can be noted these BER bounds are very close to the analytical result in the low region of average $E_{b} / N_{0}$, where the SI that results from the second MPG is insignificant, hence verifying the methods incorporated in the SAT. The effects of the SI can be seen in the high average $E_{b} / N_{0}$ region, where the BER becomes constant, regardless of the increased average $E_{b} / N_{0}$, and exhibits an irreducible error rate floor.

Curves 9 , with up-arrow markers, and 10 , with down-arrow markers show the upper and lower bounds on BER computed using the SAT when two-Rake fingers were used on the two-tap Rayleigh fading channel. It can be seen that significant gain was obtained as a result of the Rake combining.

Commonly, when considering the effects of SI, in order to obtain a tractable BER result, chip-spaced MPG delays are assumed, i.e. the relative time-delay between any received MPGs is assumed to be an integer multiple of the chip period. The PDF of SI 
based on this assumption follows a binomial distribution as demonstrated on Chapter 2 . When the spreading factor is large enough, the PDF of SI can be approximated by a Gaussian distribution, with zero-mean and variance equal to the spreading factor.

Figure 4.2.2 shows the differences between BER results estimated using the SAT and those estimated with a Gaussian model for the PDF of SI. The BER results for one Rake finger on a two-tap equal power Rayleigh fading channel were obtained with different spreading factors for simulation with 10,000 channel realizations.

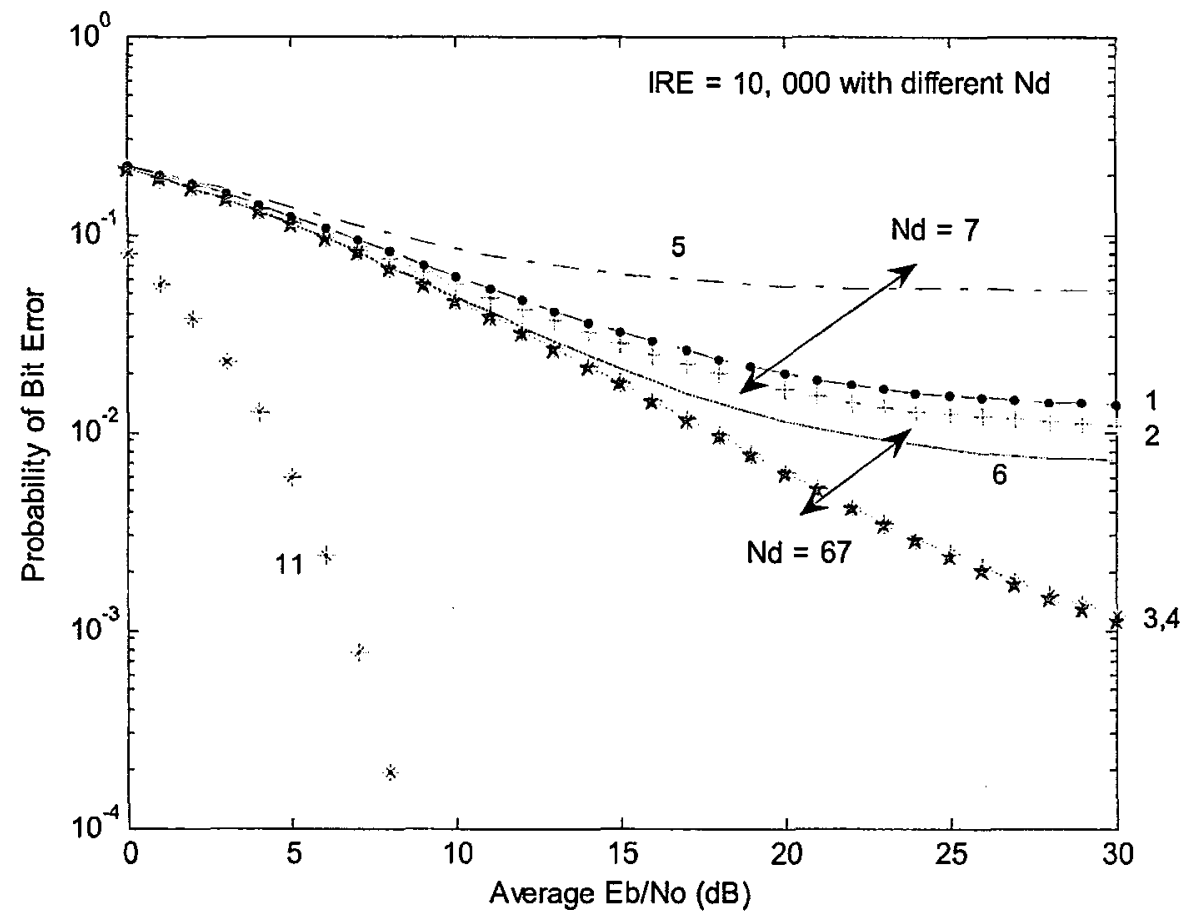

Fig.4.2.2: Comparison between BER results calculated using the SAT and BER estimated using a Gaussian model for the PDF of the SI for a Rake receiver with $\mathrm{L}=1$ and two equal powered MPGs. 
Curve 1, with the dot markers and curve 2, with the cross markers are the upper and lower bounds on BER bounds estimated using the SAT, with $N_{d}=7$. Curve 5 is the BER calculated using the Gaussian model for the PDF of SI with $N_{d}=7$, the equation is given [3] as:

$$
P_{E}=Q\left(\frac{N_{d} b \sum_{i=1}^{L}\left|h_{i}\right|^{2}}{\sqrt{\sigma_{I}^{2}+\sigma_{\text {Rake }}^{2}}}\right)
$$

where $\sigma_{\text {Rake }}^{2}$ is the variance of the noise, $\sigma_{I}^{2}$ is the variance of the SI and equals:

$$
\sigma_{I}^{2}=4 N_{d} \sum_{i=1}^{L} \sum_{j=i+1}^{L}\left|h_{i}\right|^{2}\left|h_{j}\right|^{2}+N_{d} \sum_{i=1}^{L} \sum_{j=L+1}^{K}\left|h_{i}\right|^{2}\left|h_{j}\right|^{2}
$$

It can be noted that the difference between BER results estimated using the SAT and those calculated using equation (4.2.4) with the Gaussian model for the PDF of the SI is greater for the low spreading factor in the region of practical average $E_{b} / N_{0}$. This is because the Gaussian model for the PDF of the SI is a better fit when the spreading factor is greater. However, even in the case of the higher spreading factor, the error with respect to the above-verified BER estimated with SAT is significant. If such results were used in system design, it would lead the overestimation of the required signal-to-noise ratio, resulting in lower over all cellular system spectral efficiency.

There are two sources of error that can be encountered when static BER values are averaged along a measured time series, as described in the foregoing. The first is, when the tracking of energy at a particular Rake finger is not $100 \%$ accurate. This can result in sudden drops of signal power at the finger under consideration, and yield error rate floors, where there would be none under perfect signal tracking conditions. Such errors did 
occur in the analysis of data for some street sections. This can, however, be detected by visual inspection of results. Results with this type of error were discarded. The second is, if the number of independent samples is small, resulting BER vs. average $E_{b} / N_{o}$ curves that attain an abnormally steep slope at high values of average $E_{b} / N_{o}$, giving lower BER values than those that would actually be achieved. The values of average $E_{b} / N_{o}$ at which this becomes significant depends on the statistical characteristics of the sampled data, and therefore varied from street section-to-street section in the work reported in this thesis. As a result of visual inspection, it can be reported that at values of average $E_{b} / N_{o}$ up to $15 \mathrm{~dB}$, such error occurrences were rare. 


\subsection{BER predictions for high data rate transmission}

This section reports the prediction of BERs for cases where high data rates are to be transmitted in IMT2000 bandwidths, as for example, in some anticipated IMTAdvanced, single user applications. A spreading factor of 7 is used, resulting in a transmission rate of $550 \mathrm{kbps}$. Using the SAT to accurately account for SI, the BER values were estimated from 1-sec-long time data files recorded on 27 street sections with the $T x$ at site $T x 1$ in downtown Ottawa. Fig.4.3.1 shows a typical BER result, based on data recorded when the receiver was on Kent Street, driving from Gloucester St. to Laurier Avenue. One can see that above a threshold of $-20 \mathrm{~dB}$, there were 3 received MPGs at $6 \mathrm{GHz}$ with relative powers of $0,-6.6,-14.5 \mathrm{~dB}$. Also there were 3 received MPGs at $2 \mathrm{GHz}$ with relative powers of $0,-2.2$, and $-14.1 \mathrm{~dB}$. The two strongest MPGs were used for Rake combining at both frequencies, since in both cases these had powers greater than $-7 \mathrm{~dB}$ with respect to that of the signal Rake finger 1.

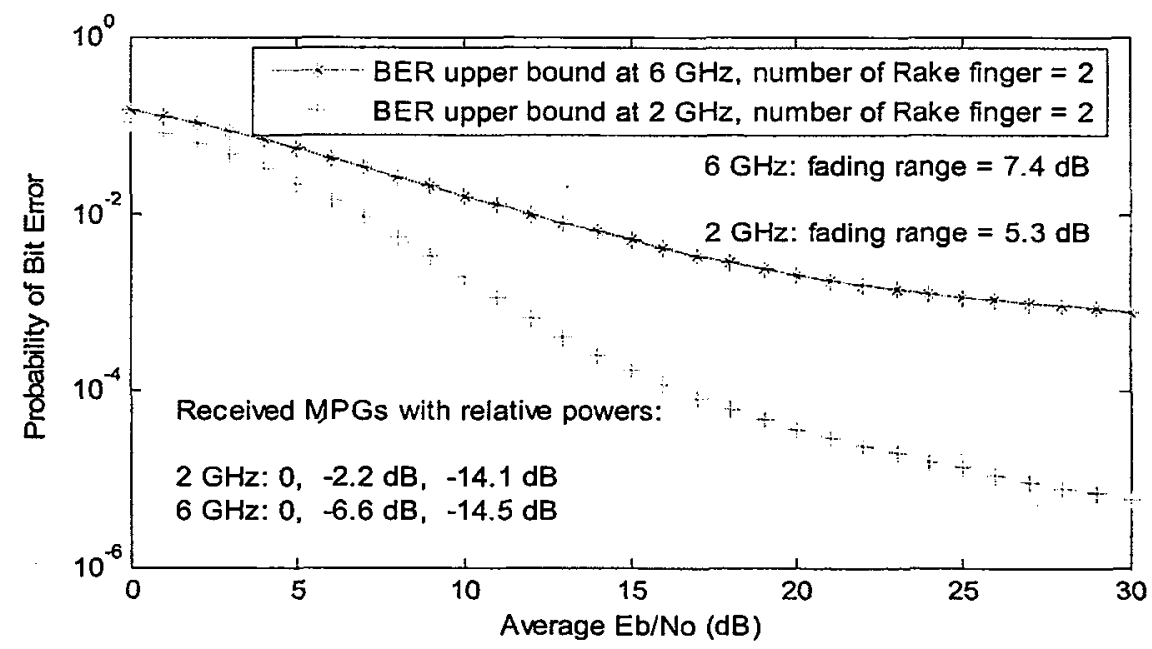

Fig.4.3.1: BER vs. Average $E_{b} / N_{o}$ results based on time series measurements on Kent Street, between Gloucester St. and Laurier Ave. with $N_{d}=7$. 
After Rake combining, the fading range of the signal presented to the Rake decision device was $7.4 \mathrm{~dB}$ at $6 \mathrm{GHz}$, and $5.3 \mathrm{~dB}$ at $2 \mathrm{GHz}$. Since the fading range of the signal is a major factor in establishing the slope of the relationship between BER and the average $E_{b} / N_{o}$, it can be predicted that in the low $E_{b} / N_{o}$ region, when $\mathrm{SI}$ is insignificant, the $6 \mathrm{GHz}$ BER curve should have a shallow slope and the $2 \mathrm{GHz}$ BER curve should have a steeper slope. In the high $E_{b} / N_{o}$ region, when SI comes significant, the BER curves at both frequencies should show irreducible error rate floors.

From Fig.4.3.1, one can see that there is a greater slope of the $2 \mathrm{GHz}$ BER curve than that of the $6 \mathrm{GHz} \mathrm{BER}$ curve in the low $E_{b} / N_{o}$ region, such as values of average $E_{b} / N_{0}$ less than $15 \mathrm{~dB}$. At values of average $E_{b} / N_{o}$ bigger than $16 \mathrm{~dB}$, the SI caused by the strong delayed MPG at $2 \mathrm{GHz}$ is significant, showing the degradation of the BER performance, and resulting in an error rate floor near $6 \times 10^{-6}$ when values of average $E_{b} / N_{o}$ are bigger than $30 \mathrm{~dB}$. The SI caused by the weaker delayed MPG at 6 $\mathrm{GHz}$ is, however, insignificant until the value of average $E_{b} / N_{o}$ is $20 \mathrm{~dB}$, and the irreducible error rate floor at $6 \mathrm{GHz}$ is near $7 \times 10^{-4}$ when values of average $E_{b} / N_{o}$ are bigger than $30 \mathrm{~dB}$.

Fig.4.3.2 shows another example of BER results estimated using the SAT from data recorded when the receiver was on Kent Street, driving from Slater St. to Albert St. Above the threshold $-20 \mathrm{~dB}$, there were 6 received MPGs at $6 \mathrm{GHz}$ with relative powers $0,-3.5,-6.7,-10.8,-16.7$, and $-19.6 \mathrm{~dB}$, and 5 received MPGs at $2 \mathrm{GHz}$ with relative powers $0,-9.8,-11.1,-14.5,-18.3 \mathrm{~dB}$. Three MPGs whose powers were greater than - 
$7 \mathrm{~dB}$ were used for rake combining at $6 \mathrm{GHz}$, while only the strongest MPG was used at 2 $\mathrm{GHz}$.

After Rake combining, the fading range of the signal presented to the receiver's decision device was $7 \mathrm{~dB}$ in both frequency bands. This means that BER curves at both frequencies should have same slope in low $E_{b} / N_{o}$ region when SI is insignificant. Because the $6 \mathrm{GHz}$ combining signal has stronger delayed MPGs, there should be stronger SI effects at $6 \mathrm{GHz}$ in high $E_{b} / N_{o}$ region.

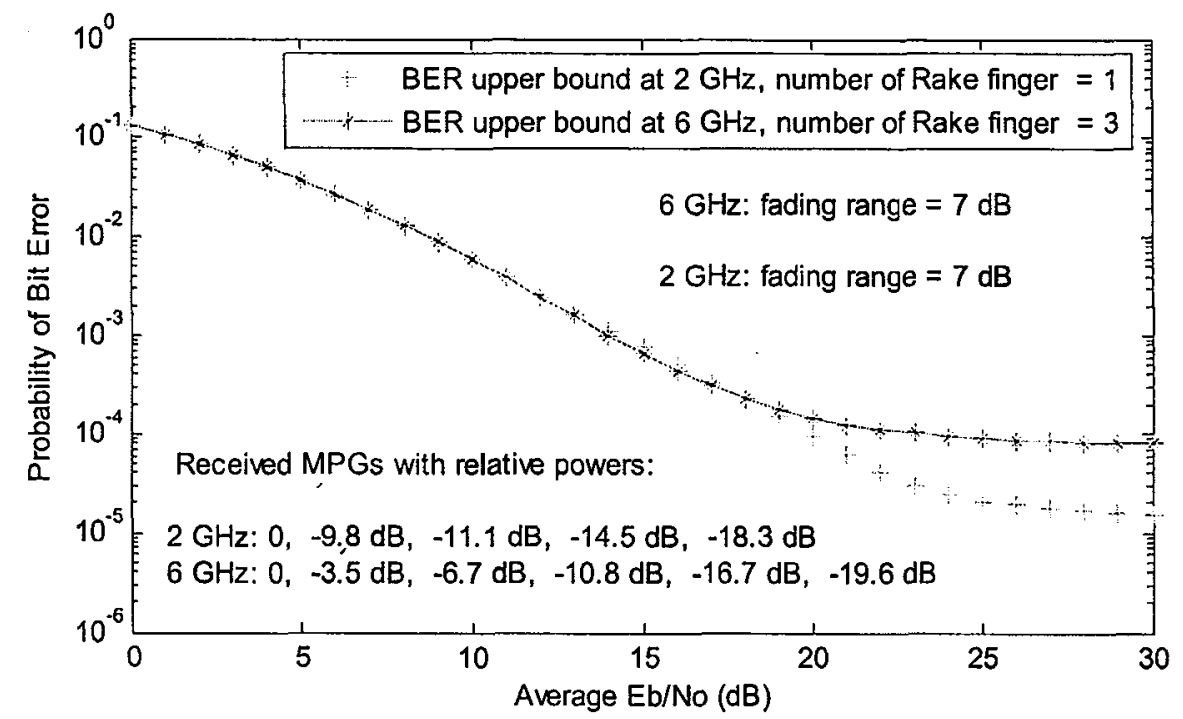

Fig.4.3.2: BER vs. Average $E_{b} / N_{o}$ results based on time series measurements on Kent Street, between Slater St. and Albert St. with $N_{d}=7$.

In Fig.4.3.2, it is shown that for the values of average $E_{b} / N_{o}$ up to about $17 \mathrm{~dB}$, the $2 \mathrm{GHz}$ and $6 \mathrm{GHz}$ signals have same BER curves. At values of average $E_{b} / N_{\circ}$ greater than $19 \mathrm{~dB}$, the SI effects caused by the two delayed strongest MPGs at $6 \mathrm{GHz}$ become significant, and can be seen to have caused more severe performance degradation, resulting in a higher irreducible error rate floor at $6 \mathrm{GHz}$ at the BER value about $8 \times 10^{-5}$ 
when values of average $E_{b} / N_{o}$ are bigger than $22 \mathrm{~dB}$. The SI caused by the weaker delayed MPG at $2 \mathrm{GHz}$ doesn't become significant until the value of average $E_{b} / N_{0}$ equal to $27 \mathrm{~dB}$ and the error floor $1.5 \times 10^{-5}$ is reached at a value of average $E_{b} / N_{o} 30$ dB.

One result of practical interest that can be obtained from BER vs average $E_{b} / N_{o}$ results, such as those discussed in the foregoing is the value of average $E_{b} / N_{o}$ that is required to achieve a specified BER performance. ECDFs for the average $E_{b} / N_{o}$ required achieving a BER of $10^{-3}$ in the 2 and $6 \mathrm{GHz}$ bands are shown in Fig.4.3.3.

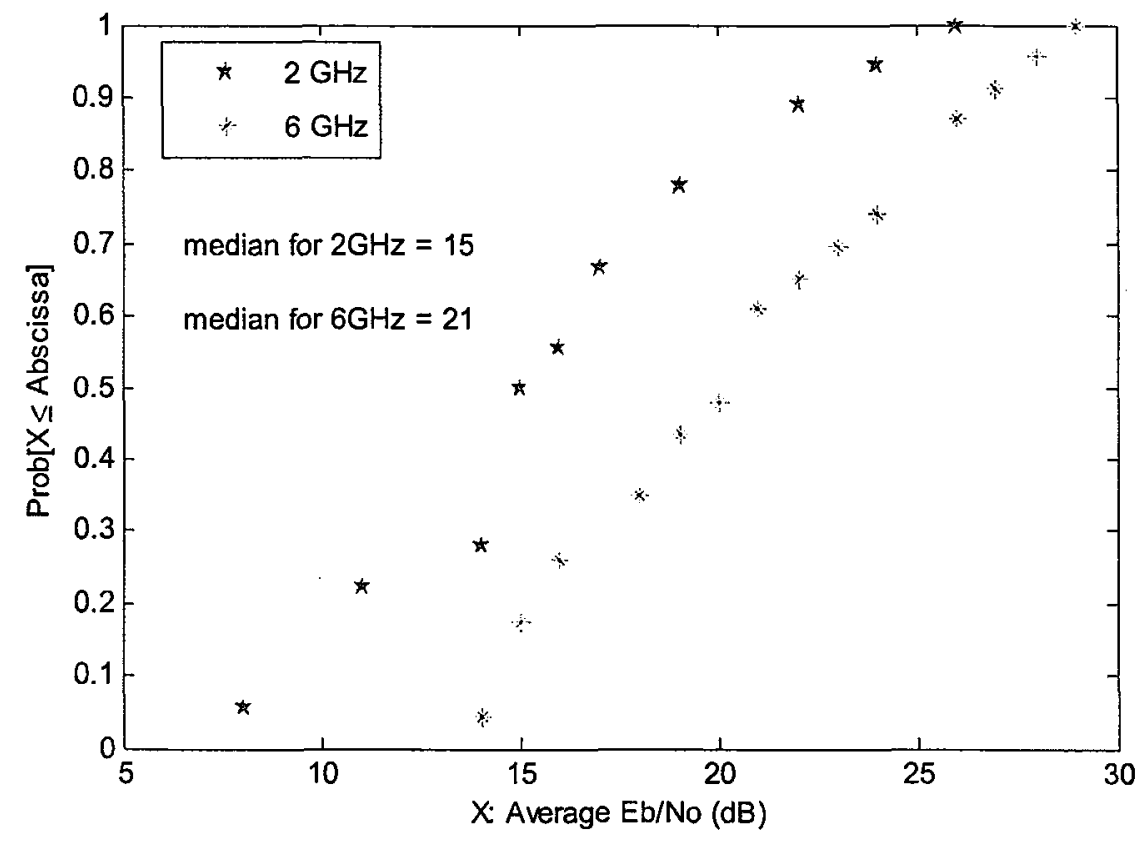

Fig.4.3.3: ECDFs of the average $E_{b} / N_{o}$ required to achieve $\mathrm{BER}=10^{-3}$ at 2 and $6 \mathrm{GHz}$.

In considering the results in Fig.4.3.3, it should be noted that at $2 \mathrm{GHz}$, there were 9 street sections, i.e. $33 \%$ of the measured street sections, where a BER of $10^{-3}$ could not 
be achieved because of the onset of an irreducible error rate floor caused by SI. At $6 \mathrm{GHz}$, there were 4 street sections, i.e. $15 \%$ of the measured street sections on which a BER of $10^{-3}$ could not be achieved. It is believed that there were more such street sections at 2 $\mathrm{GHz}$ because of greater SI in that band caused by higher-powered delayed multipath components, as evidences by the slightly greater RMS delay spreads at $2 \mathrm{GHz}$.

From Fig.4.3.3, it can be seen that, in the cases where it would be possible to achieve a BER of $1 \mathrm{E}-03$ without channel protection, the median of average $E_{b} / N_{o}$ at $2 \mathrm{GHz}$ is 15 $\mathrm{dB}$, while that at $6 \mathrm{GHz}$ is $21 \mathrm{~dB}$.

From the BER relationships estimated from data measured on different street sections, through setting the average $E_{b} / N_{o}$ to $15 \mathrm{~dB}$ and $20 \mathrm{~dB}$, one can determine the achievable BER performance, given the cited values of $E_{b} / N_{o}$ at both frequencies, as shown by the ECDFs in Fig.4.3.4 and Fig.4.3.5.

From Fig.4.3.4, with average $E_{b} / N_{o}$ of $15 \mathrm{~dB}$, the median at $2 \mathrm{GHz}$ is $2.4 \mathrm{E}-03$, and the median at $6 \mathrm{GHz}$ is $4.5 \mathrm{E}-03$; from Fig.4.3.5, the median BER at $2 \mathrm{GHz}=8.7 \mathrm{E}-04$, and the median of BER at $6 \mathrm{GHz}=1.3 \mathrm{E}-03$. Thus, at the same signal to noise ratio, better bit error performance would be achievable at $2 \mathrm{GHz}$ than at $6 \mathrm{GHz}$. This is compatible with the results in Chapter 3, which show that there is a higher probability of fades at 6 GHz. 


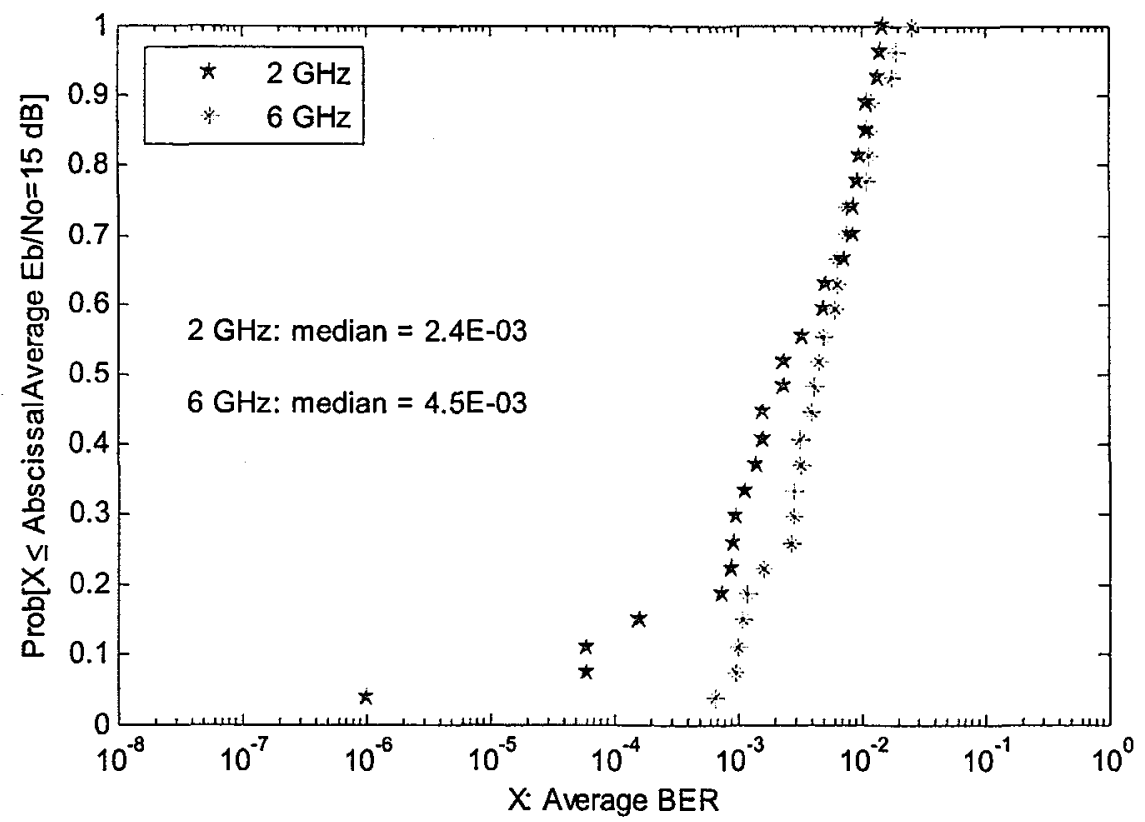

Fig.4.3.4: ECDFs of BER at 2 and $6 \mathrm{GHz}$ if an average $E_{b} / N_{o}$ of $15 \mathrm{~dB}$ is available on all 27 street sections.

Fig.4.3.4 shows that when average $E_{b} / N_{o}$ is set to be $15 \mathrm{~dB}, 30 \%$ of the street sections at $2 \mathrm{GHz}$ can achieve $10^{-3}$ of BER performance, and only $15 \%$ of the street sections at $6 \mathrm{GHz}$ can achieve the same BER performance. This indicates that channel protection would be required in both bands for adequate performance with a spreading factor of 7 (i.e. a data rate of $550 \mathrm{kbps}$ with IMT2000 bandwidths). It is also conjectured based on these results that the costs associated with such protection could be greater at 6 $\mathrm{GHz}$, since error performance on unprotected channels is worse in that band. 


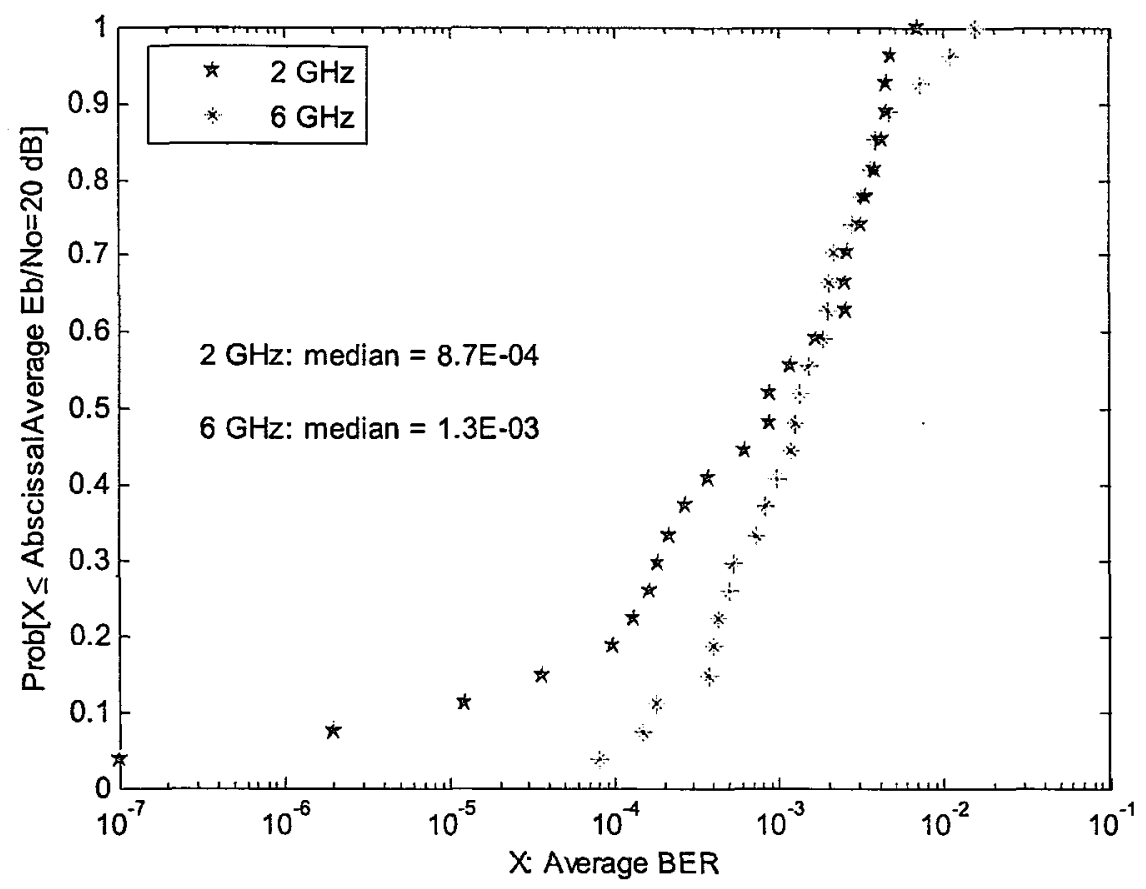

Fig.4.3.5: ECDFs of BER at 2 and $6 \mathrm{GHz}$ if an average $E_{b} / N_{o}$ of $20 \mathrm{~dB}$ is available on all 27 street sections.

Fig.4.3.5 shows that when the average $E_{b} / N_{o}$ is $20 \mathrm{~dB}$, a BER of $10^{-3}$ could be achieved on about $55 \%$ of the measured street sections at $2 \mathrm{GHz}$, and $40 \%$ at $6 \mathrm{GHz}$. For the BER of $10^{-4}$, only about $20 \%$ of the street sections at $2 \mathrm{GHz}$ can be achieved and meanwhile only $5 \%$ at $6 \mathrm{GHz}$. 


\subsection{BER predictions for low data rate transmission}

This section reports the prediction of BER performance on the measured street sections for transmission with a high spreading factor $\left(N_{d}=128\right)$, which leads to data rates of around $30 \mathrm{kbps}$ within IMT2000 bandwidths. Recall from Chapter 3 that median RMS delay spreads were about $300 \mathrm{~ns}$. This means that, if a GWSSUS channel model is assumed, coherence bandwidths would be around $3 \mathrm{MHz}$, and SI should be insignificant for data rates in the range under consideration in this section. To reduce computation times, estimation of the effects of SI, as previously described, was therefore omitted in BER calculations leading to results reported in this section.

\subsubsection{BER predictions from data recorded with $\mathrm{Tx}$ at site $\mathrm{Tx} 2$}

Average BER values were estimated from 1-sec-long time data files recorded on 48 street sections in downtown Ottawa. Fig.4.4.1 shows a typical average BER result for transmission with a high spreading factor $N_{d}=128$. The associated SRE time series was recorded when the receiver was on Laurier Avenue, driving from Lyon St. to Kent St. 


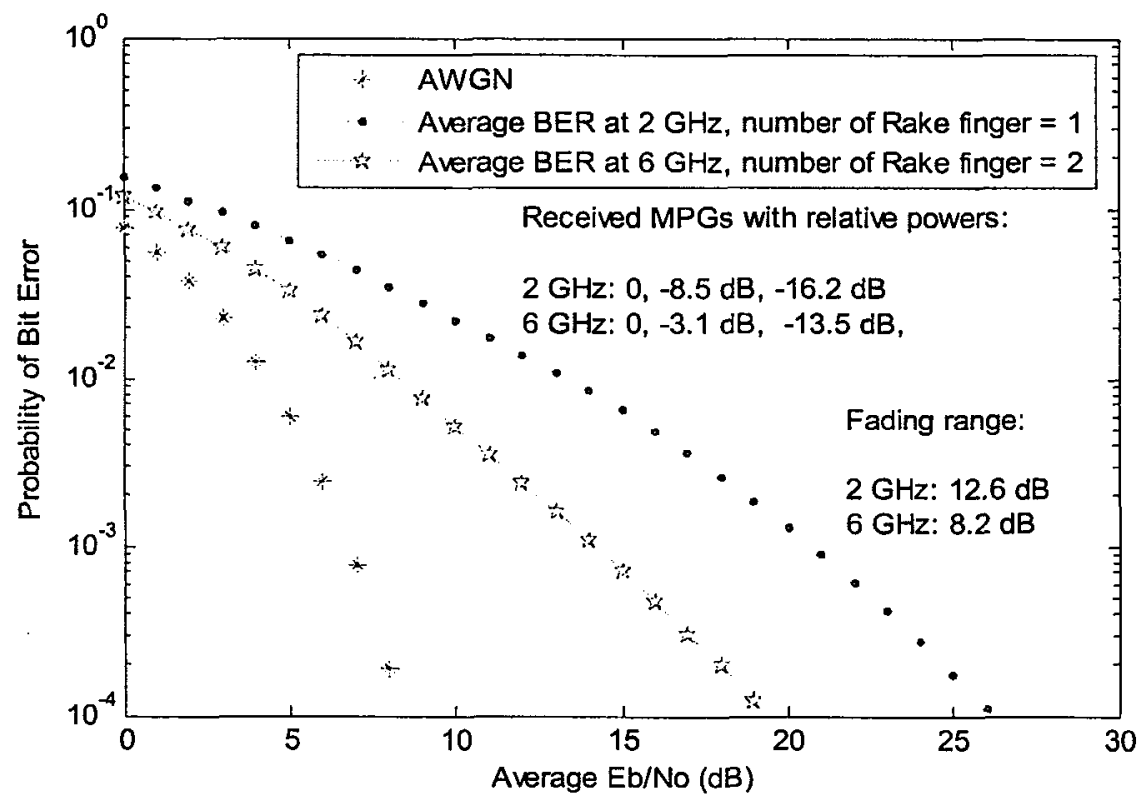

Fig.4.4.1: BER vs. Average $E_{b} / N_{o}$ based on time series measurements on Laurier Avenue, driving from Lyon St. to Kent St. with $N_{d}=128$.

From the text in Fig.4.4.1, one can note that above the threshold $-20 \mathrm{~dB}$, there were 3 received MPGs at $2 \mathrm{GHz}$ with relative powers $0,-8.5,-16.2 \mathrm{~dB}$, and 3 received MPGs at $6 \mathrm{GHz}$ with relative powers $0,-3.1,-13.5 \mathrm{~dB}$. Because the $2^{\text {nd }} \mathrm{MPG}$ has a power greater than $-7 \mathrm{~dB}$ compared with the strongest MPG, two Rake fingers were used to predict the BER performance at $6 \mathrm{GHz}$, and only one Rake finger for the BER prediction at $2 \mathrm{GHz}$. The fading range of the signal presented to the Rake decision device was $12.6 \mathrm{~dB}$ at 2 $\mathrm{GHz}$, and $8.2 \mathrm{~dB}$ at $6 \mathrm{GHz}$. This greater range of fading at $2 \mathrm{GHz}$ signal, led to the shallower slope of $2 \mathrm{GHz}$ BER curve.

The value of average $E_{b} / N_{0}$ that is required to achieve a specified BER performance $\left(10^{-3}\right)$ was studied for LOS and NLOS street sections separately, and the results are shown in Fig.4.4.2. 


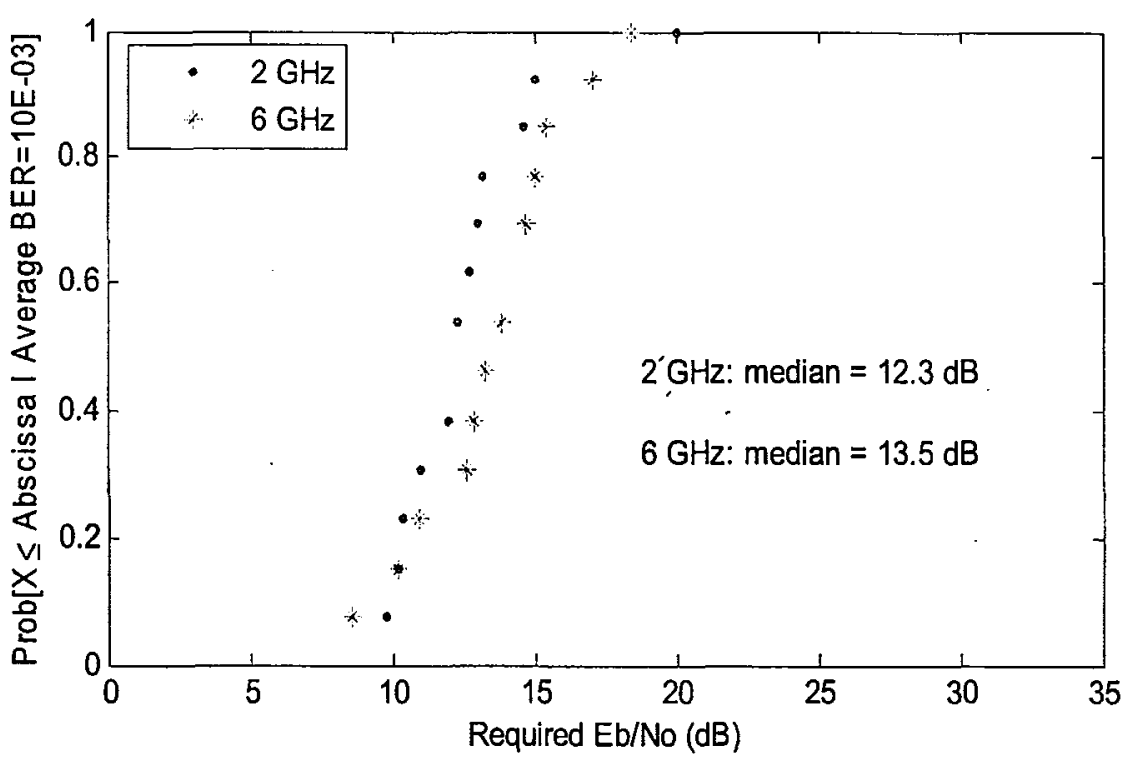

(a) Required $E_{b} / N_{o}$ for LOS.

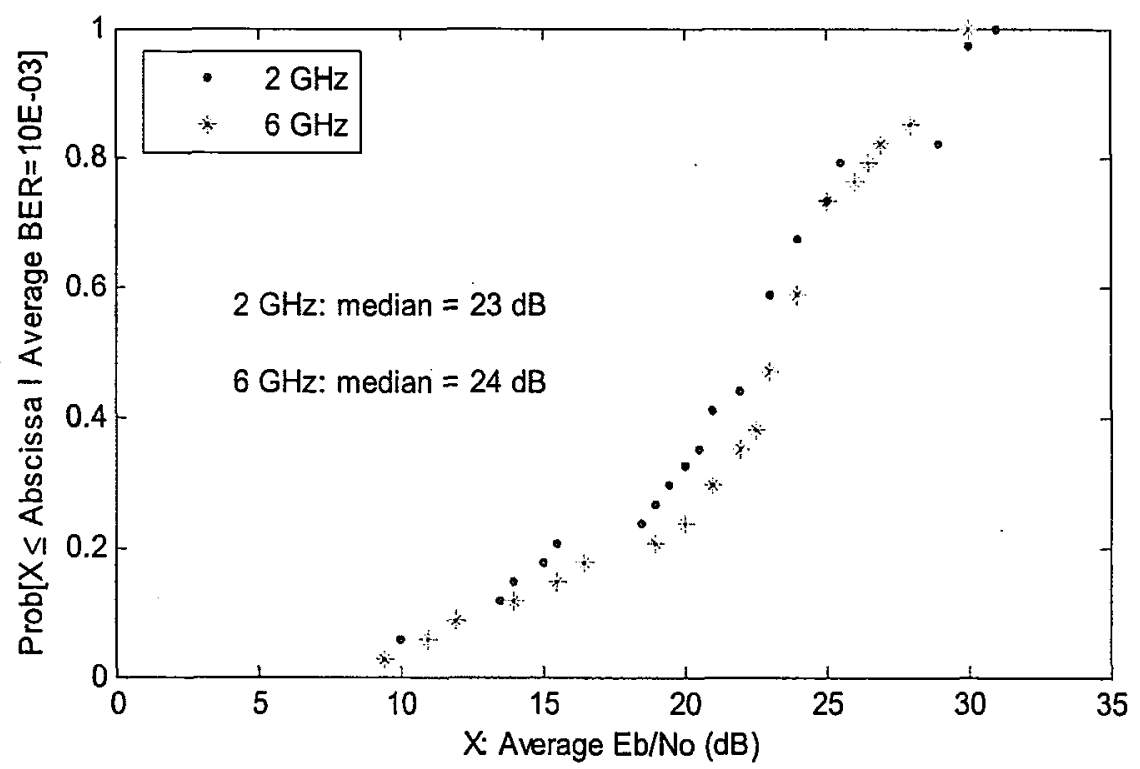

(b) Required $E_{b} / N_{o}$ for NLOS.

Fig.4.4.2: ECDFs for the average value of $E_{b} / N_{o}$ required achieving a BER of $10^{-3}$ at 2 and $6 \mathrm{GHz}$ with the Tx on Kent Street. 
Fig.4.4.2 shows it would be possible to achieve a BER of $10^{-3}$ without channel protection for all street sections. On LOS street sections, the median of average $E_{b} / N_{o}$ values at $2 \mathrm{GHz}$ is $12.3 \mathrm{~dB}$, while that at $6 \mathrm{GHz}$ is $13.5 \mathrm{~dB}$. On NLOS street sections, the median of average $E_{b} / N_{\mathrm{o}}$ at $2 \mathrm{GHz}$ is $23 \mathrm{~dB}$, while that at $6 \mathrm{GHz}$ is $24 \mathrm{~dB}$. It should be noted that the above-reported values have an associated estimation error that is function of the number of independent data used to compile the results. Because the data pool for these measurements is small, this estimation error is such that a statistically significant comparison of the 2 and $6 \mathrm{GHz}$ results cannot be reported.

The results of an alternative study, shown in Fig.4.4.3 and Fig.4.4.4, were obtained through setting the average $E_{b} / N_{0}$ to $15 \mathrm{~dB}$ or $20 \mathrm{~dB}$ to determine the achievable BER performance, given the cited values of $E_{b} / N_{o}$ at both frequencies.

From the text in Fig.4.4.3, one can see that on LOS street sections, when the average $E_{b} / N_{o}$ is $15 \mathrm{~dB}$, the median of $\mathrm{BER}$ values at $2 \mathrm{GHz}$ is $1.6 \mathrm{E}-04$, while that at $6 \mathrm{GHz}$ is 3.9E-04. On NLOS street sections, the median of BER values at $2 \mathrm{GHz}$ is $6.4 \mathrm{E}-03$, while that at $6 \mathrm{GHz}$ is $7.1 \mathrm{E}-03$. The figure also shows that when the average $E_{b} / N_{o}$ is set to 15 $\mathrm{dB}$, in LOS cases, conditions on $92 \%$ of the street sections at $2 \mathrm{GHz}$ would yield BER values of $10^{-3}$, while the same performance could be achieved on only $77 \%$ of the measured street sections at $6 \mathrm{GHz}$. On NLOS street sections, conditions on only $18 \%$ of the street sections at $2 \mathrm{GHz}$ would lead to a BER of $10^{-3}$, while those on $12 \%$ of the street sections at $6 \mathrm{GHz}$ would lead to this performance. This indicates that for conditions such as those that existed when the measurement system $\mathrm{Tx}$ was at site $\mathrm{Tx} 2$, on Kent Street, even with a spreading factor of 128 (i.e. a low data rate of $30 \mathrm{kbps}$ with IMT2000 
bandwidths), channel protection is needed in NLOS at both frequencies. It is also conjectured based on these results that the costs associated with such protection could be greater at $6 \mathrm{GHz}$, since error performance on unprotected channels is worse in that band.

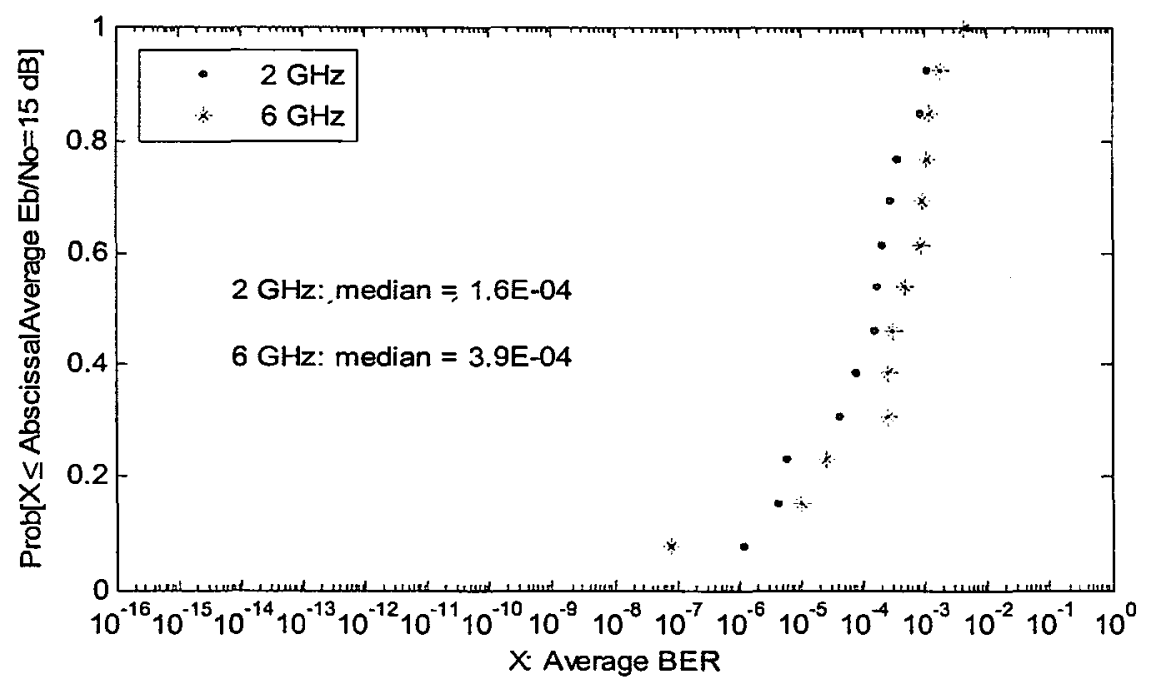

(a) BER for 14 LOS street sections.

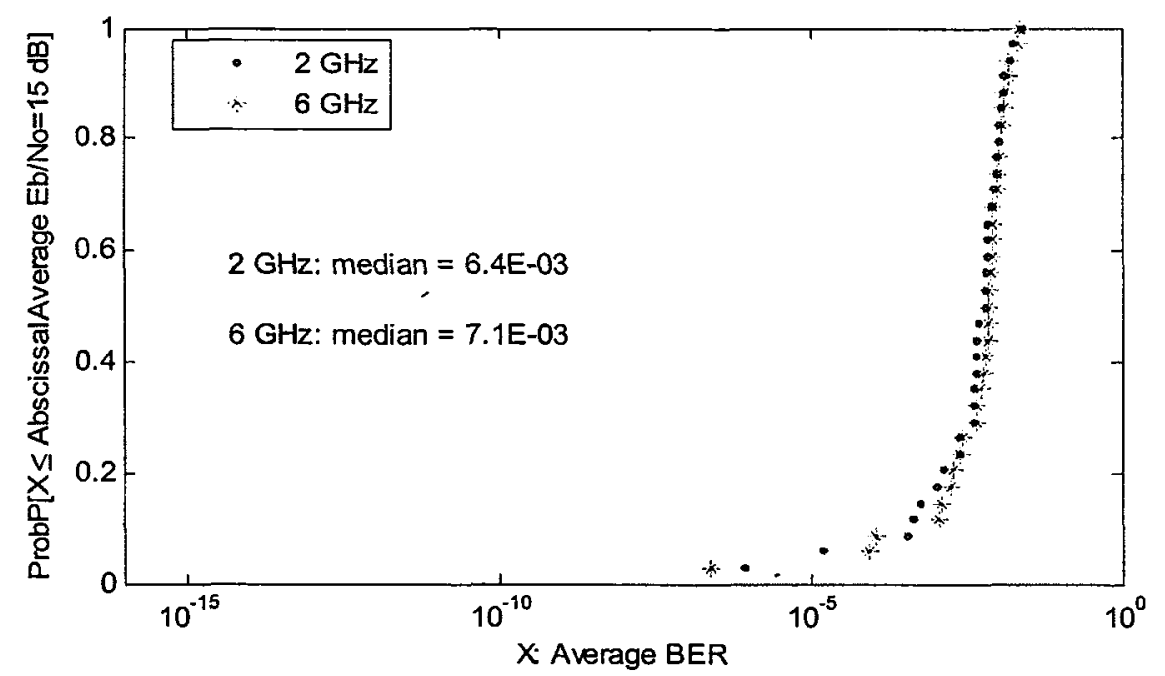

(b) BER for 34 NLOS street sections.

Fig.4.4.3: ECDFs of BER at 2 and $6 \mathrm{GHz}$ if an average $E_{b} / N_{o}$ of $15 \mathrm{~dB}$ is available at both frequencies with Tx on Kent Street. 


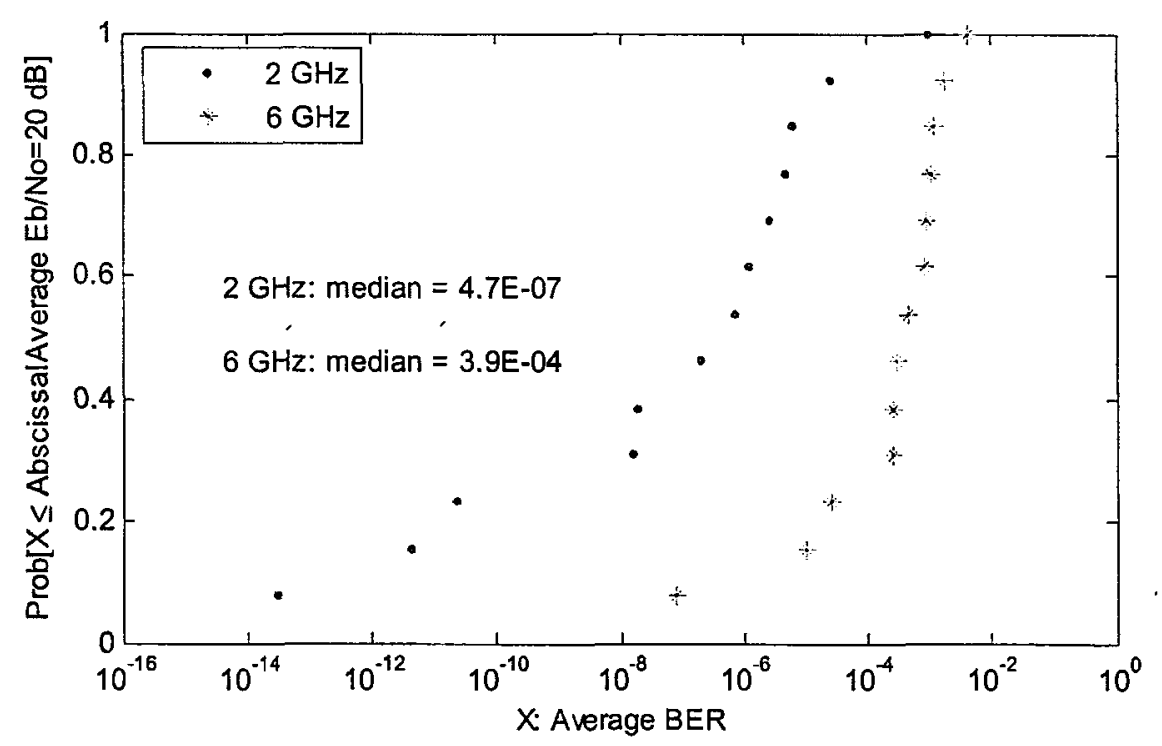

(a) BER for 14 LOS street sections.

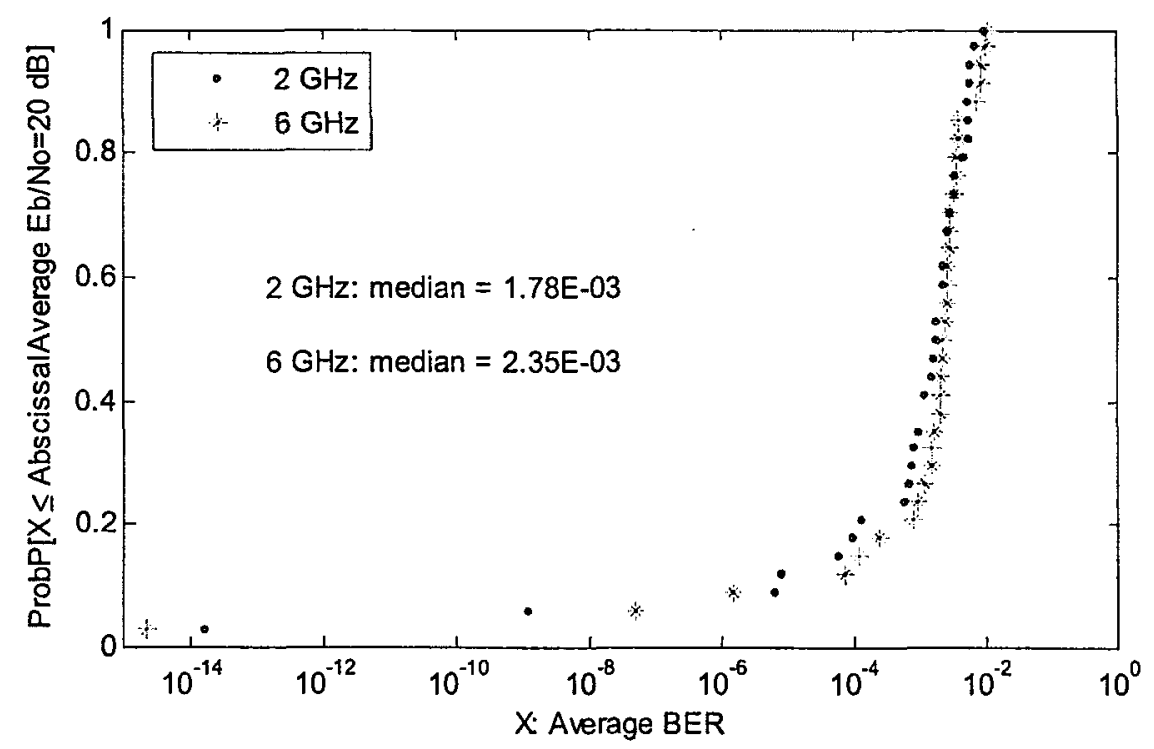

(b) BER for 34 NLOS street sections.

Fig.4.4.4: ECDFs of BER at 2 and $6 \mathrm{GHz}$ if an average $E_{b} / N_{o}$ of $20 \mathrm{~dB}$ is available at both frequencies with Tx on Kent Street. 
From the text in Fig.4.4.4, one can see that on LOS street sections, when the average $E_{b} / N_{o}$ is $20 \mathrm{~dB}$, the median of BER values at $2 \mathrm{GHz}$ is $4.7 \mathrm{E}-07$, while that at $6 \mathrm{GHz}$ is 3.9E-04. On NLOS street sections, the median of BER values at $2 \mathrm{GHz}$ is $1.8 \mathrm{E}-03$, while that at $6 \mathrm{GHz}$ is $2.3 \mathrm{E}-03$. The figure shows that if the average $E_{b} / N_{o}$ is $20 \mathrm{~dB}$, on LOS street sections, a BER of $10^{-3}$ could be achieved on nearly all street sections at $2 \mathrm{GHz}$, whereas this BER would be achievable on only $77 \%$ of the street sections at $6 \mathrm{GHz}$.. On NLOS street sections, conditions on about $35 \%$ of the street sections at $2 \mathrm{GHz}$ would lead to the achievement of a BER of $10^{-3}$, while those on about $26 \%$ of the street sections would result in the same performance at $6 \mathrm{GHz}$.

\subsubsection{BER predictions from data recorded with $\mathrm{Tx}$ at site $\mathrm{Tx} 1$}

Average BER values were estimated from 1-sec-long time data files recorded on 147 street sections in downtown Ottawa. Observations and statistics concerning these results are reported here. Fig. 4.4.5 shows a typical average BER result for transmission with a high spreading factor of 128 . The associated SRE time series was recorded when the receiver was on the intersection between Bank Street and Slater Street. 


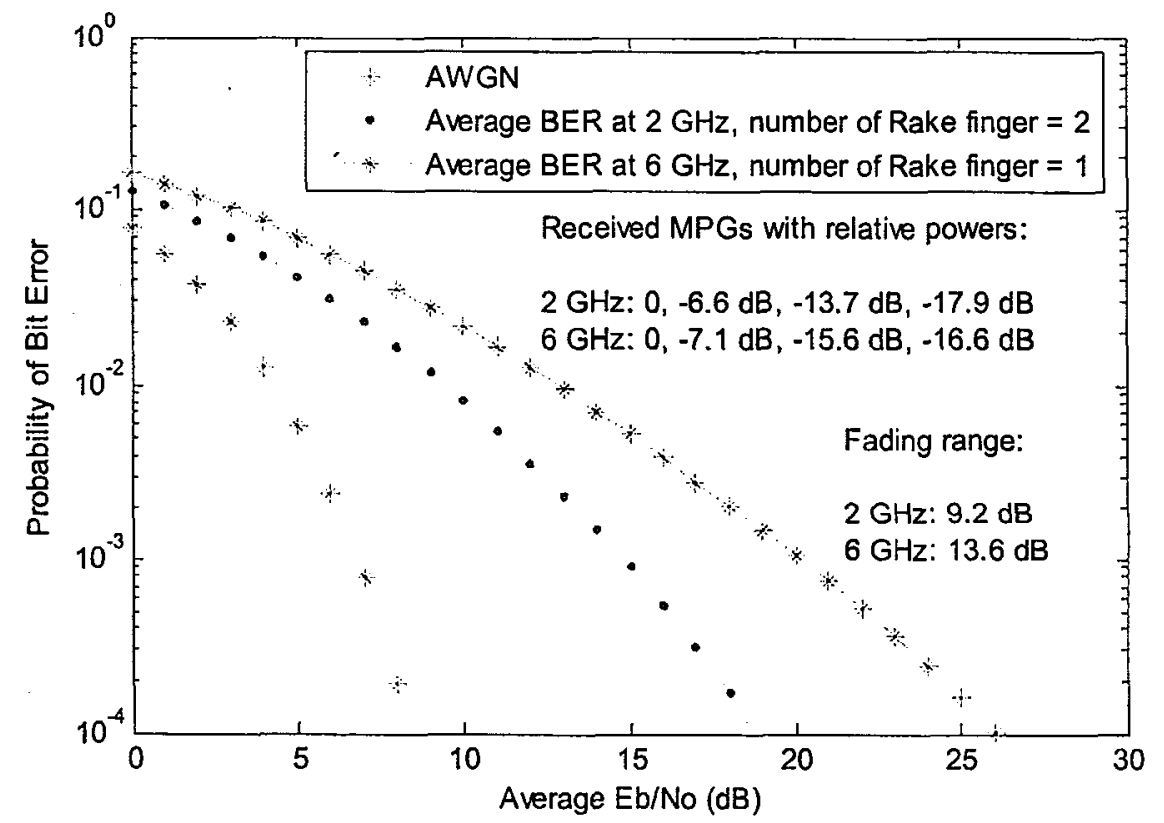

Fig.4.4.5: BER vs. Average $E_{b} / N_{o}$ results based on time series measurements on the intersection between Bank Street and Slater Street with $N_{d}=128$.

From the text in Fig.4.4.5, one can note that above the threshold $-20 \mathrm{~dB}$, there were 4 received MPGs at $2 \mathrm{GHz}$ with relative powers $0,-6.6,-13.7$ and $-17.9 \mathrm{~dB}$. There were 4 received MPGs at $6 \mathrm{GHz}$ with relative powers $0,-7.1,-15.6$, and $-16.6 \mathrm{~dB}$. Two Rake fingers were used for the prediction of BER performance at $2 \mathrm{GHz}$, and one Rake finger at $6 \mathrm{GHz}$. The fading range of the signal presented to the Rake decision device was 9.2 $\mathrm{dB}$ at $2 \mathrm{GHz}$, and $13.6 \mathrm{~dB}$ at $6 \mathrm{GHz}$. This greater range of fading at $6 \mathrm{GHz}$ signal, led to the shallower slope of $6 \mathrm{GHz}$ BER curve.

The value of average $E_{b} / N_{o}$ that is required to achieve a specified BER performance $\left(10^{-3}\right)$ is studied for LOS and NLOS street sections separately, and the results are shown in Fig.4.4.6. 


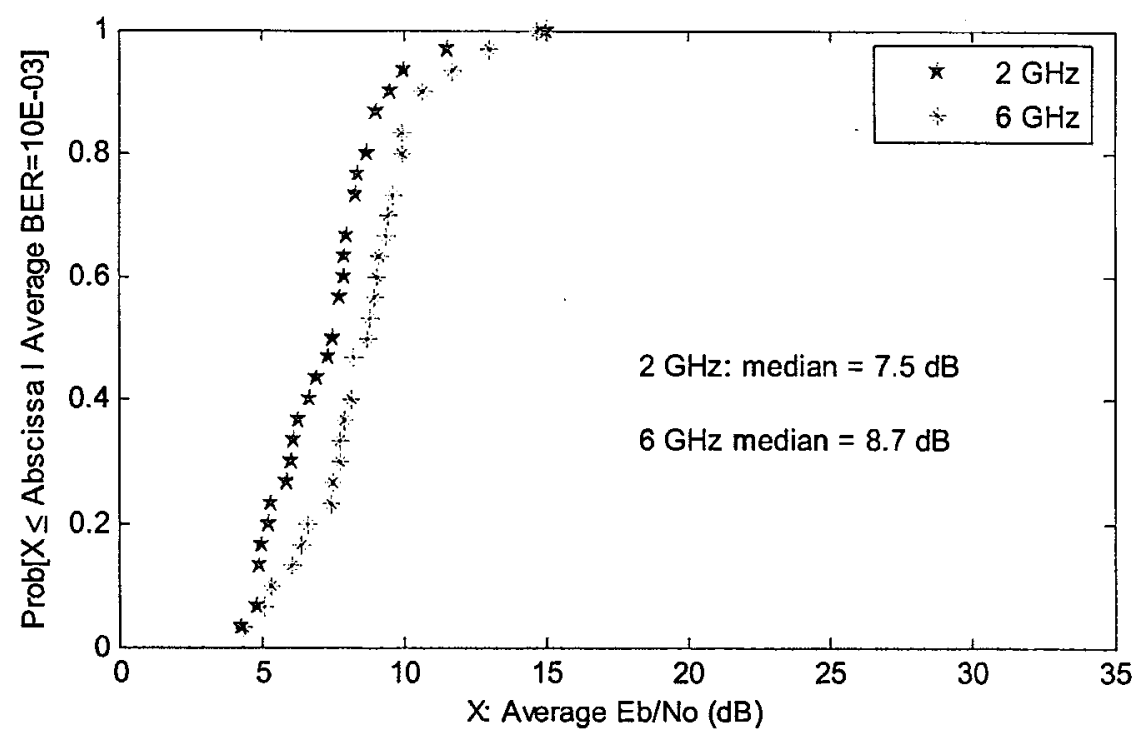

(a) Required $E_{b} / N_{o}$ for 30 LOS street sections.

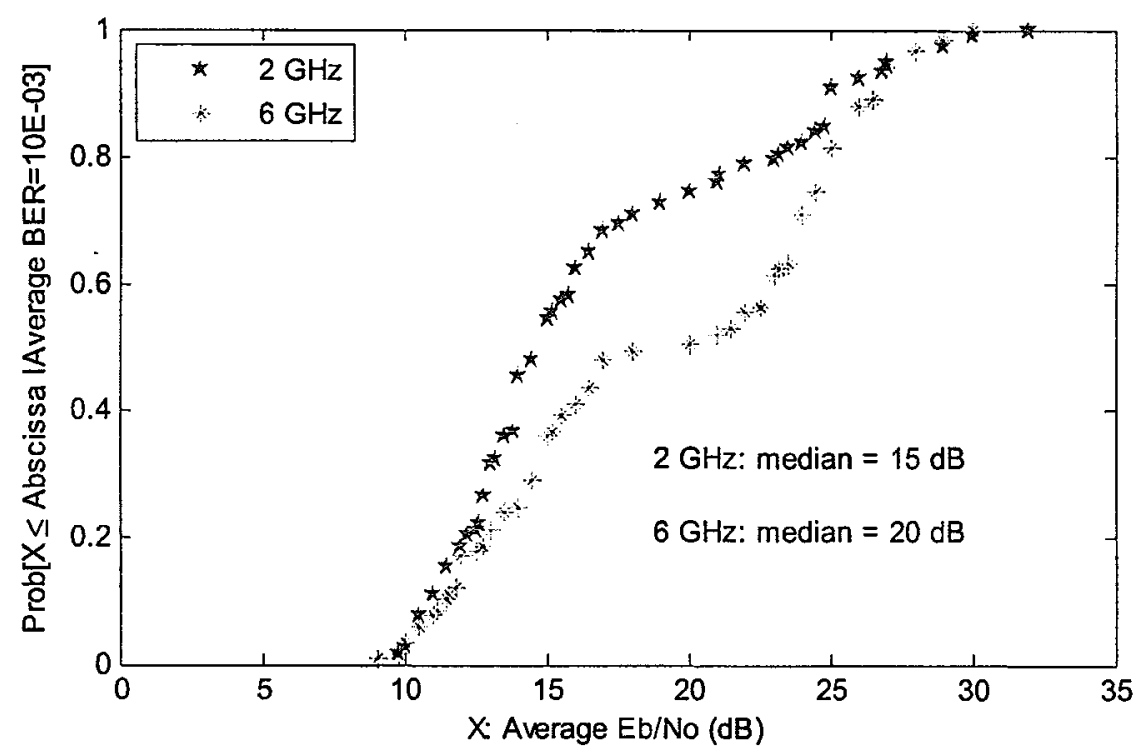

(b) Required $E_{b} / N_{o}$ for 117 NLOS street sections.

Fig.4.4.6: ECDFs for the average value of $E_{b} / N_{o}$ required achieving a BER of $10^{-3}$ at 2 and $6 \mathrm{GHz}$ with $\mathrm{Tx}$ on Laurier Street. 
From the text in Fig.4.4.6, it can be seen that on LOS street sections in order to achieve a BER of $10^{-3}$ without channel protection, the median of average $E_{b} / N_{o}$ values at $2 \mathrm{GHz}$ is $7.5 \mathrm{~dB}$, and that at $6 \mathrm{GHz}$ is $8.7 \mathrm{~dB}$. On NLOS street sections in order to achieve the same BER performance, the median of average $E_{b} / N_{o}$ at $2 \mathrm{GHz}$ is $15 \mathrm{~dB}$, and that at $6 \mathrm{GHz}$ is $20 \mathrm{~dB}$. The figure shows it would be possible to achieve a BER of $10^{-3}$ without channel protection for all street sections with the $\mathrm{Tx}$ at site $\mathrm{Tx} 1$, on Laurier Ave. Fig.4.4.7 and Fig.4.4.8 show the achievable BERs on unprotected channels if the average value of $E_{b} / N_{o}$ were $15 \mathrm{~dB}$ and $20 \mathrm{~dB}$, respectively.

On LOS street sections, when the average $E_{b} / N_{o}$ is $15 \mathrm{~dB}$, the median of BER values at $2 \mathrm{GHz}$ is $1 \mathrm{E}-04$, while that at $6 \mathrm{GHz}$ is $5.1 \mathrm{E}-04$. On NLOS street sections, the median of BER values at $2 \mathrm{GHz}$ is $1 \mathrm{E}-03$, while that at $6 \mathrm{GHz}$ is $3.6 \mathrm{E}-03$. The figure also shows that when the average $E_{b} / N_{0}$ is $15 \mathrm{~dB}$, on LOS street sections, a BER of $10^{-3}$ would be achievable on $87 \%$ of the street sections at $2 \mathrm{GHz}$, and on $67 \%$ of the street sections at $6 \mathrm{GHz}$. In NLOS cases, this performance could be achieved on only $50 \%$ of street sections at $2 \mathrm{GHz}$ and $33 \%$ of street sections at $6 \mathrm{GHz}$. These results show that BER performance is slightly better compared with those estimated with the SAT for a spreading factor of 7 , in which case a BER of $10^{-3}$ would be achievable on $30 \%$ of the street sections at $2 \mathrm{GHz}$ and only $15 \%$ of the street sections at $6 \mathrm{GHz}$ when average $E_{b} / N_{o}$ is $15 \mathrm{~dB}$. Channel protection in NLOS cases would still be required even with a spreading factor of 128 (i.e. a low data rate of $30 \mathrm{kbps}$ with IMT2000 bandwidths) in both frequency bands. 


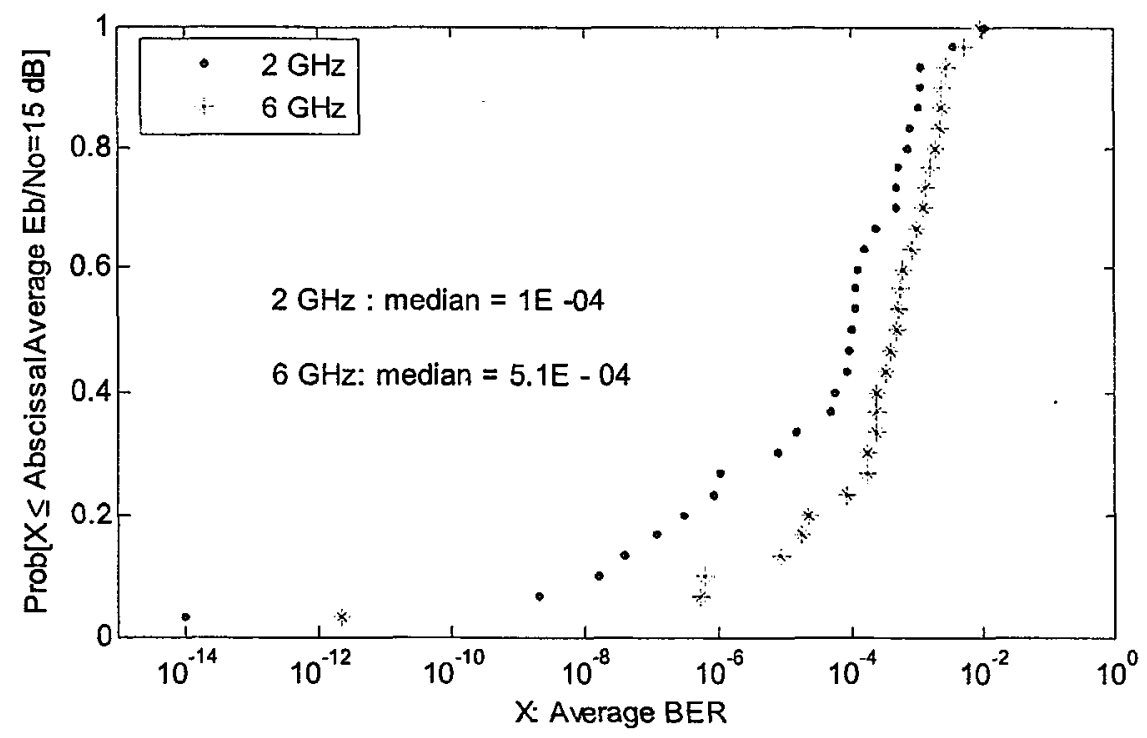

(a) BER for 30 LOS street sections.

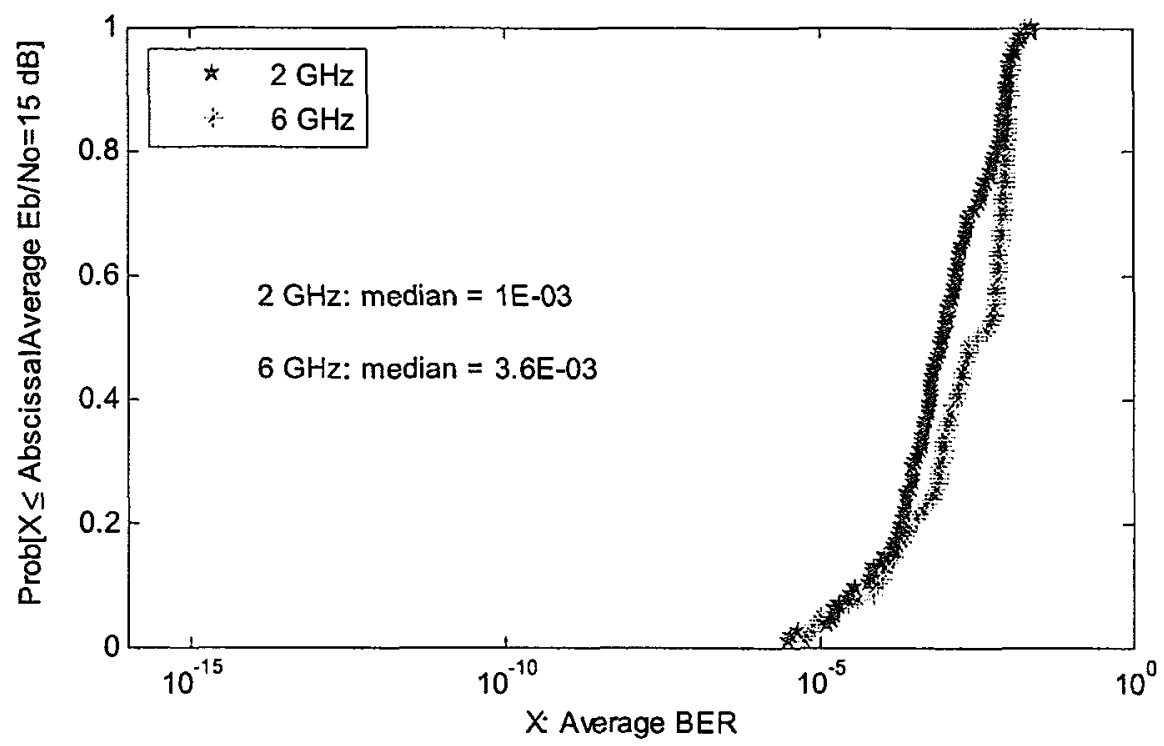

(b) BER for 117 NLOS street sections

Fig.4.4.7: ECDFs of BER if an average $E_{b} / N_{o}$ of $15 \mathrm{~dB}$ is available at both frequencies with the Tx on Laurier Avenue. 


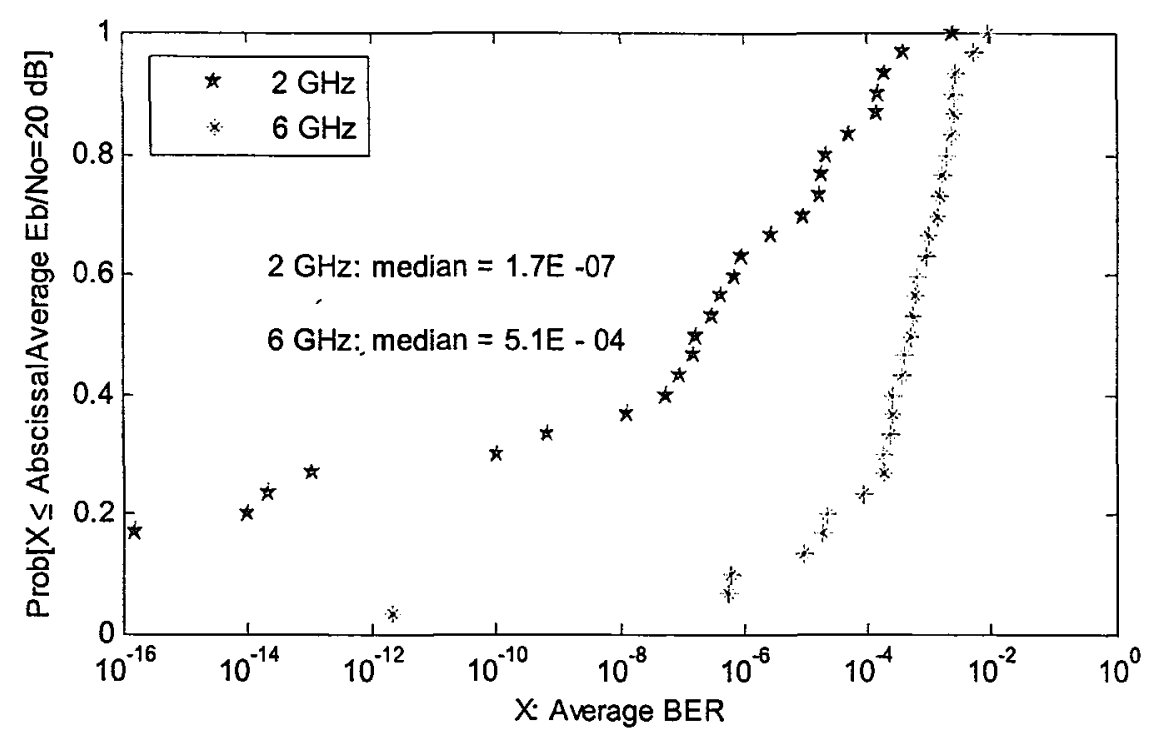

(a) BER for 30 LOS street sections.

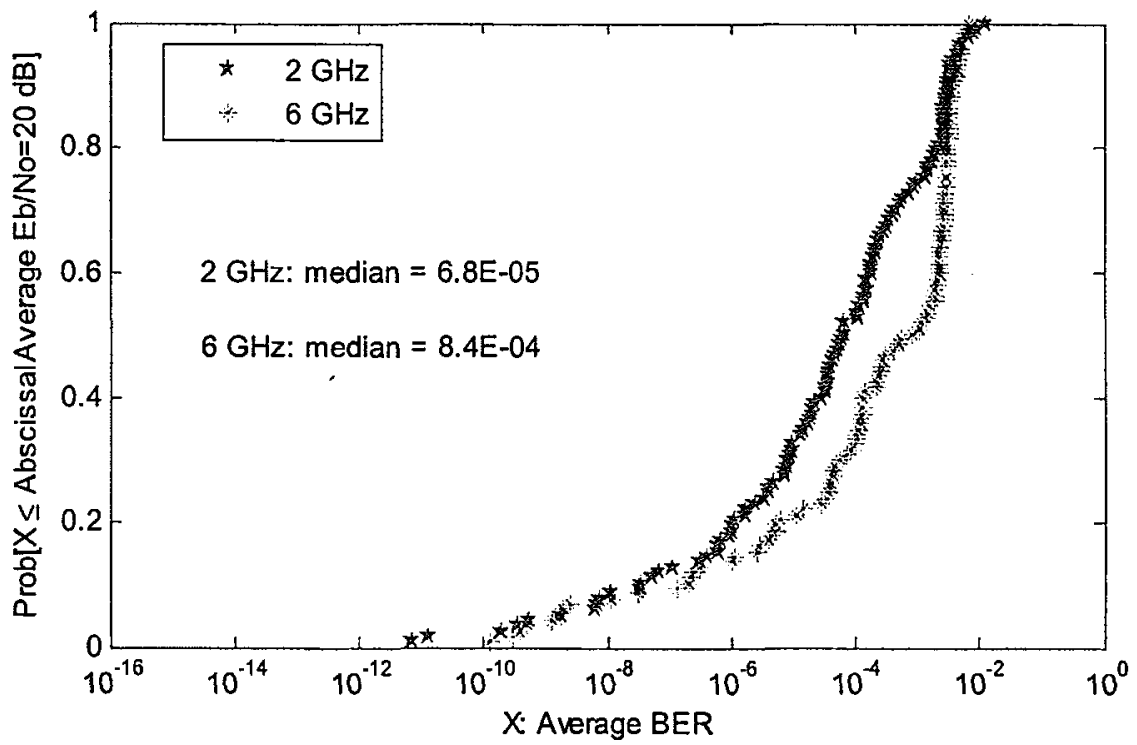

(b) BER for 117 NLOS street sections

Fig.4.4.8: ECDFs of BER if an average $E_{b} / N_{o}$ of $20 \mathrm{~dB}$ is available at both frequencies with the Tx on Laurier Avenue. 


\subsection{Conclusions}

The SAT reported by Adnani, which accurately accounts for SI, and avoids the use of simplified fading channel models through time series analysis of measured radio channel data was used for the prediction of bit-error performance for transmission with a low spreading factor of 7 (high data rate) on DSSS radio links with IMT2000 bandwidths in both the 2 and $6 \mathrm{GHz}$ frequency bands. The objective was to determine if additional technology would be needed if IMTAdvanced (i.e. higher data rate) services were to be provided by simple changes in the operating parameters (spreading factor) and centre frequency of current IMT2000 systems.

Results show that due to the onset of error rate floors caused by SI, a BER of $10^{-3}$ could not be achieved on $33 \%$ of the measured street sections at $2 \mathrm{GHz}$, and on 15 $\%$ of the measured street sections at $6 \mathrm{GHz}$. This number was greater at $2 \mathrm{GHz}$ because greater temporal dispersion in that band resulted in more significant levels of SI. Given reasonable average values of $E_{b} / N_{o}$, such as $15 \mathrm{~dB}$ or $20 \mathrm{~dB}$, could be maintained throughout a microcell, a BER of $10^{-3}$ could be achieved at $2 \mathrm{GHz}$ on $30 \%$ and $55 \%$, respectively, of the measured street sections, and only on $15 \%$ and $40 \%$, respectively, of the measured street sections at $6 \mathrm{GHz}$. It appears, therefore that, without any channel protection, the BER performance would be slightly better at $2 \mathrm{GHz}$. This indicates that channel protection would be required in both bands for adequate performance with a spreading factor of 7 (i.e. a data rate of $550 \mathrm{kbps}$ with IMT2000 bandwidths). It can also can be speculated that channel protection, needed to achieve acceptable coverage at a BER of $10^{-3}$ would be more costly at $6 \mathrm{GHz}$, since error performance on unprotected channels is worse in that band. 
Low data rate cases, of interest in the study of whether current IMT2000 systems can simply be transplanted to higher frequency bands to achieve greater capacities where there is spectral overcrowding were also studied. Average BER performance in IMT2000 bandwidths with a high spreading factor of 128 , giving a data rate of $30 \mathrm{kbps}$ was evaluated. The analyses showed that the performance was slightly worse at $6 \mathrm{GHz}$, and the results can be significantly different for different base station locations. Given a reasonable average $E_{b} / N_{b}$ of $15 \mathrm{~dB}$ or $20 \mathrm{~dB}$ throughout a microcell, the details on percentages of street sections where a BER of $10^{-3}$ could achieve with different location of Tx are listed in Table 4.1.

Table 4.1: Percentage of street sections where the achievement of a BER of $10^{-3}$ would be possible with the different $T x$ locations in downtown Ottawa.

\begin{tabular}{|c|c|c|c|c|c|}
\hline \multirow{2}{*}{$\begin{array}{l}\text { Average } \\
\text { BER }\end{array}$} & \multirow[b]{2}{*}{ Frequency } & \multicolumn{2}{|c|}{ Average $\mathrm{Eb} / \mathrm{No}=15 \mathrm{~dB}$} & \multicolumn{2}{|c|}{ Average $\mathrm{Eb} / \mathrm{No}=20 \mathrm{~dB}$} \\
\hline & & LOS & NLOS & LOS & NLOS \\
\hline \multirow{2}{*}{$10^{-3}$} & $2 \mathrm{GHz}$ & $92 \%$ & $18 \%$ & $100 \%$ & $35 \%$ \\
\hline & $6 \mathrm{GHz}$ & $77 \%$ & $12 \%$ & $78 \%$ & $26 \%$ \\
\hline
\end{tabular}

(a) Percentage of 47 street sections with the $\mathrm{Tx}$ at site $\mathrm{Tx} 1$ on Kent St.

\begin{tabular}{|l|l|l|l|l|l|}
\hline \multirow{2}{*}{$\begin{array}{l}\text { Average } \\
\text { BER }\end{array}$} & \multirow{2}{*}{ Frequency } & \multicolumn{2}{|l|}{ Average Eb/No $=15 \mathrm{~dB}$} & \multicolumn{2}{l|}{ Average Eb/No = 20 dB } \\
\cline { 3 - 6 } & LOS & NLOS & LOS & NLOS \\
\hline \multirow{2}{*}{$10^{-3}$} & $2 \mathrm{GHz}$ & $87 \%$ & $50 \%$ & $97 \%$ & $74 \%$ \\
\cline { 2 - 6 } & $6 \mathrm{GHz}$ & $67 \%$ & $33 \%$ & $67 \%$ & $51 \%$ \\
\hline
\end{tabular}

(b) Percentage of 147 street sections with the Tx at site $\mathrm{Tx} 2$ on Laurier Ave.

Generally, BER performance is better at $2 \mathrm{GHz}$ than that at $6 \mathrm{GHz}$ because at high frequency, the phase of signal changes more rapidly, leading to frequent more fading at 6 GHz. The BER performance would be different with different base station locations, also with the same base station location, BER performance for low data rate services (high 
spreading factors) would be better compared with those achievable with high data rates (low spreading factors) . Channel protection in NLOS would still be required even with a low data rate of $30 \mathrm{kbps}$ at both frequencies and the costs associated with such protection could be greater at $6 \mathrm{GHz}$, since error performance on unprotected channels is worse in that band. 


\section{Chapter 5}

\section{Summary, conclusions, and recommendations}

\section{for further work}

Two subprojects were carried out in the course of this thesis. The first involved the comparison of channel parameters at $2 \mathrm{GHz}$ and $6 \mathrm{GHz}$. Quasi-simultaneous wideband propagation measurements at two frequencies, one in each of these bands, were conducted. Measurement equipment, measurements and data analyses methods were described in Chapter 3. Channel parameters and characteristics, such as average power delay profiles, fading statistics of multipath groups (MPGs), each comprised of multipath signal components received at excess delays separated by less than the resolution time of the measurement system and RMS delay spreads were estimated from data measured in both frequency bands during three sets of experiments in downtown Ottawa. Interest in the dynamics of MPGs arises because the energy in MPGs would constitute that received at different finger positions in a Rake receiver. Results showed that the fading distributions of MPGs vary significantly with the location of a microcellular Base Station when the density and height of buildings is not uniform. Envelope fading among MPGs having powers within $7 \mathrm{~dB}$ of the strongest MPG exhibited low cross correlations, showing potential advantages in Rake combining to achieve better BER performance. Typically up to three MPGs were available for Rake combining. In the 1-sec-long time 
series analysed during the work of this thesis, ECDFs of fading ranges for MPGs after Rake combining showed that the range between the tenth and ninetieth percentiles of envelope fading distributions is greater at $6 \mathrm{GHz}$ compared with that at $2 \mathrm{GHz}$. This is considered to be the result of the fact that wavelength is shorter at $6 \mathrm{GHz}$ and thus, within any given time and space interval, phase relationships that result in a fade are more probable.

RMS delay spreads were also estimated. Results showed that these channel parameters can also vary significantly with base station location. Experiments that were conducted with the simulated base station transmitter at Kent Street, between Gloucester St. and Laurier Ave. showed that, under LOS conditions, RMS delay spreads at $2 \mathrm{GHz}$ are approximately the same as those at $6 \mathrm{GHz}$. The median at $2 \mathrm{GHz}$ was $118 \mathrm{~ns}$, and that at $6 \mathrm{GHz}$ was $116 \mathrm{~ns}$. However, RMS delay spreads were found to be greater at $2 \mathrm{GHz}$ under NLOS conditions, with the median being $200 \mathrm{~ns}$ at $2 \mathrm{GHz}$ and $160 \mathrm{~ns}$ at $6 \mathrm{GHz}$, respectively. Experiments that were conducted with the transmitter at a simulated base station at Laurier Avenue, between Kent St. and Bank St resulted in RMS delay spreads at $2 \mathrm{GHz}$ that are greater under both LOS and NLOS. The medians at $2 \mathrm{GHz}$ were $202 \mathrm{~ns}$ and $329 \mathrm{~ns}$ for LOS and NLOS, and those at $6 \mathrm{GHz}$ were $173 \mathrm{~ns}$ and $295 \mathrm{~ns}$.

The second subproject entailed application of a semi-analytical technique (SAT) for Rake receiver BER prediction on DSSS radio links, as reported by Adnani [3]. This technique accurately accounts for SI, and avoids the use of simplified fading channel models through time series analysis of measured radio channel data. This method was used for the prediction of bit-error performance achievable on the measured street sections. For the assessment of radio links in IMTAdvanced systems with high data rates, 
a low spreading factor $\left(N_{d}=7\right)$ was assumed for operation within IMT2000 bandwidths (3-5 MHz) in both the 2 and $6 \mathrm{GHz}$ frequency bands. The objective was to determine if additional technology would be needed if IMTAdvanced services were to be provided by simple changes in the operating parameters and centre frequency of current IMT2000 systems.

\section{Conclusions}

Results from the analysis of error performance showed that, in conformance with the observation of greater RMS delay spreads, $2 \mathrm{GHz}$ operation would suffer more significant SI than operations at $6 \mathrm{GHz}$. However, as a result of the lower probability of fades at $2 \mathrm{GHz}$, given reasonable average values of $E_{b} / N_{o}$, without any channel protection, the BER performance would be slightly better at $2 \mathrm{GHz}$. Under NLOS conditions, when the transmitter was on Laurier Avenue, between Kent St. and Bank St., with any practical average $E_{b} / N_{o}$ up to $30 \mathrm{~dB}$, the analysis results showed that the error performance of $10^{-3}$ could not be achieved on $33 \%$ of the measured street sections at 2 $\mathrm{GHz}$, and on $15 \%$ at $6 \mathrm{GHz}$. The number was greater at $2 \mathrm{GHz}$ because there was more significant SI at $2 \mathrm{GHz}$. For the remaining street sections, given the average $E_{b} / N_{o}$ of 15 $\mathrm{dB}$, the BER of $10^{-3}$ could be achieved on $30 \%$ at $2 \mathrm{GHz}$, and on $15 \%$ at $6 \mathrm{GHz}$. If the average $E_{b} / N_{o}$ was increased to $20 \mathrm{~dB}$, the same performance could be achieved on 55 $\%$ of the measured street sections at $2 \mathrm{GHz}$ and $40 \%$ at $6 \mathrm{GHz}$. Results indicated that channel protection would be required in both bands to achieve bit error rates of $10^{-3}$ or 
better with a spreading factor of 7 (i.e. a data rate of $550 \mathrm{kbps}$ with IMT2000 bandwidths). It is speculated, however, that channel protection, needed to achieve acceptable coverage would be more costly at $6 \mathrm{GHz}$, since error performance on unprotected channels is worse in that band for any given average value of $E_{b} / N_{o}$. In addition, based on previous work [2], approximately $10 \mathrm{~dB}$ more transmit power would be required in the 6 $\mathrm{GHz}$ band to achieve these average values of $E_{b} / N_{0}$.

Low data rate cases, of interest in the study of whether current IMT2000 systems can simply be transplanted to higher frequency bands to achieve greater capacities where there is spectral overcrowding were also studied. Average BER performance in IMT2000 bandwidths with a high spreading factor of 128 , giving a data rate of $30 \mathrm{kbps}$ was evaluated. Results again showed that error performance would be slightly poorer in the higher frequency band. Error performance changed significantly with different simulated base station (transmitter) locations. Under NLOS conditions, when the transmitter was on Kent Street, between Gloucester St. and Laurier St., given an average $E_{b} / N_{\circ}$ of $15 \mathrm{~dB}$, a BER of $10^{-3}$ would be achievable only on $18 \%$ of the measured street sections at $2 \mathrm{GHz}$, and $12 \%$ at $6 \mathrm{GHz}$. Increasing the average $E_{b} / N_{0}$ to $20 \mathrm{~dB}$, the same performance would be achievable on $35 \%$ and $26 \%$ of the measured street sections at $2 \mathrm{GHz}$ and $6 \mathrm{GHz}$, respectively. When the transmitter was on Laurier Avenue, between Kent St. and Bank St., with the average $E_{b} / N_{o}$ of $15 \mathrm{~dB}$, the performance of BER of $10^{-3}$ would be achievable on $50 \%$ at $2 \mathrm{GHz}$, and $33 \%$ at $6 \mathrm{GHz}$. With an average $E_{b} / N_{o}$ of $20 \mathrm{~dB}$, the same performance would be achievable on $74 \%$ and $51 \%$ of the measured street sections at 2 and $6 \mathrm{GHz}$, respectively. It can be concluded that with any practical average $E_{b} / N_{o}$, 
channel protection in NLOS conditions would still be required to achieve a BER of $10^{-3}$ on all street sections, even with a high spreading factor of 128 (i.e. a low data rate of $30 \mathrm{kbps}$ with IMT2000 bandwidths) at both frequencies. It can therefore be concluded that, in addition to the requirement for more transmit powers at $6 \mathrm{GHz}$, there would be increased costs associated with realising the same performance on DSSS microcellular mobile radio links at $6 \mathrm{GHz}$ as that achievable on the same links at $2 \mathrm{GHz}$. It is therefore considered to be probable that proportionate performance degradation, with respect to the performance achievable at $2 \mathrm{GHz}$, would be suffered as a result of operation in any other frequency band between 2 and $6 \mathrm{GHz}$.

\section{Recommendations for further work}

There are other issues, related to the objectives of the project that could be considered in refining conclusions that result from this thesis:

- Channel estimation was assumed to be error free in the work of this thesis. Improvements could be made by realistically accounting for channel estimation errors

- Data from only 27 street sections were analysed using the SAT for predictions with low spreading factor (high data rates) because of lengthy processing times. If the associated MATLAB codes can be made more efficient, or coupled with a "C" program, more data should be analysed for greater statistical significance of results.

- Application of the SAT to analyse channel data appropriate to different RF bandwidths is a logical next step. The channel sounder used for work reported in 
this thesis was also, as part of another project, used to make similar quasisimultaneous 2 and $6 \mathrm{GHz}$ band microcell measurements with an RF bandwidth of $100 \mathrm{MHz}$ (50Mchips/s chip rate). Resulting data could be filtered to represent channel conditions over different RF bandwidths, and analysed, as in this thesis, to predict DSSS link performance to investigate tradeoffs between RF bandwidth and spreading factor in the achievement of higher data rate transmission.

- Methods, such as channel coding and equalisation to mitigate SI when lower spreading factors are used could be conducted, followed by a cost/benefit analysis including consideration of bandwidth/spreading factor tradeoffs;

- Since frequencies in the range $400 \mathrm{MHz}$ - to - $6 \mathrm{GHz}$ are being considered for the expansion of mobile services, the study reported here could be repeated with quasi simultaneous measurements at $2 \mathrm{GHz}$ and a lower frequency (say between 400 and $600 \mathrm{MHz})$. 


\section{References:}

[1] J. G. Proakis, "Digital Communications". Book. 4th edition McGraw-Hill 2000.

[2] R.J.C. Bultitude, T. C.W. Schenk, et. al., "A Propagation -Measurement-Based Evaluation of Channel Characteristics and Models Pertinent to the Expansion of Mobile Radio Systems to Frequencies beyond $2 \mathrm{GHz}$ ". Accepted for publication in IEEE Trans, Veh, Technol, spring 2007.

[3] N. Adnani, "Rake receiver performance in Direct Sequence Spread Spectrum Systems Operating at High Data Rates." Ph.D. Thesis. Carleton University, Ottawa, Canada, May 2004.

[4] T. S. Rappaport, "Wireless Communications: Principles and Practice." Prentice-Hall, NJ, 1996.

[5] G.L. Stuber, "Principles of Mobile Communication." Kluwer Academic Publishers, MA, 2nd ed. Feb. 2001.

[6] J. S. Lehnert and M.B. Pursley, "Error Probabilities for Binary Direct-Sequence Spread-Spectrum Communications with Random Signature Sequences." IEEE Trans. On Commun., vol. com-35, no.1, Jan. 1987.

[7] M. Pukkila, "Channel Estimation Modeling.” Nokia Research Center, Dec. 2000.

[8] S. Jo, J. Choi, "Modified Leaky LMS Algorithm for Channel Estimation in DSCDMA Systems." IEEE Comm Letters. Vol. 6, No. 5, May 2002.

[9] S. Coleri, M. Ergen, et. al., "A Study of Channel Estimation in OFDM Systems." IEEE 2002. 
[10] D. C. Cox, "Delay Doppler Characteristics of Multipath Propagation at $910 \mathrm{MHz}$ in a Suburban Mobile Radio Environment," IEEE Trans ., Antennas and Propagation, pp.625-635, Sep.1972.

[11] D. C. Cox, "910 MHz Urban Mobile Radio Propagation: Multipath Characteristics in New York City," IEEE Trans., vol. com-21, no.11, pp.1188-1194, Nov. 1973.

[12] D. C. Cox, "Distributions of Multipath Delay Spread and Average Excess Delay for $910 \mathrm{MHz}$ Urban Mobile Radio Paths," Oct. 1974.

[13] R. J. C. Bultitude, G. K. Bedal, "Propagation Characteristics on Microcellular Urban Mobile Radio Channels at $910 \mathrm{MHz}$." IEEE Journal on Selected Areas in Communs. vol.7, no.1, January 1989.

[14] G. Durgin, T. S. Rappaport, et. al., "Radio Path Loss and Penetration Loss Measurements in and around Homes and Trees at 5.85 GHz." IEEE, 1998.

[15] K. L. Blackard, T. S. Rappaport, et. al., "Path Loss and Delay Spread Models as Functions of Antenna Height for Microcellular System Design." IEEE, 1992.

[16] K. Kalliola, P. Vainikainen et. al., "Directional Radio Channel Measurements at Mobile Station in Different Radio Environments at $2.15 \mathrm{GHz}$." EPMCC, Vienna, 20th -22nd, February 2001.

[17] H. Boujemaa, M. Siala, "On the Rake Receiver Performance." IEEE Veh. Tech. Conf., vol.3 pp. 1483-1488, Sep. 2000.

[18] D.J. Torrieri, "Performance of Direct Sequence Systems with long pseudo noise sequences." IEEE J. Sel. Areas Commun., vol. 10, pp. 770-78, May 1992.

[19] K. Cheun, "Performance of Direct-Sequence Spread-Spectrum RAKE Receivers with Random Spreading Sequences." IEEE Trans. Commun., vol 45 No. 9 Sep.1997. 
[20] K. C. Hwang and K. B. Lee, "Performance of DS/CDMA Systems for High Data Rate Services wit Low Processing Gain." IEEE 49th Veh. Technol. Conf., vol 3 pp. 2264-2267, 1999.

[21] M. Guenach and L. Vandendorps, "Downlink Performance Analysis of a BPSKbased WCDMA using conventional Rake Receivers with Channel Estimation." IEEE J. Sel. Areas Commun., vol.19 (11), pp.2165-2176, Nov, 2001.

[22] R.F. Linfield, R.W. Hubbard, L.E. Pratt, "Transmission channel characterization by impulse response measurements". NTIS Report. Aug. 1976.

[23] W. J. Dixon and F. J. Massey, "Introduction to Statistical Analysis." $3^{\text {rd }}$ ed. New York, McGraw-Hill, 1969. 


\section{Appendix:}

\section{Semi - Analytical Technique (SAT) Review}




\section{The PDF of $I_{2,1}$}

Recall from the equation (4.1.5) in Chapter 4, the cross correlation of two randomly shifted binary sequences is presented as:

$$
W_{2,1}=\int_{\tau_{1}}^{T_{d}+\tau_{1}} b\left(t-\tau_{2}\right) a\left(t-\tau_{2}\right) a\left(t-\tau_{1}\right) d t
$$

Following [3], the above equation can be written as

$$
W_{2,1}=b_{\left\lfloor/ T_{d}\right\rfloor\left\lfloor\tau_{2,1 / 1} / T_{d}\right\rfloor-1} R_{a}\left(\tau_{2,1}-\left\lfloor\tau_{2,1} / T_{d}\right\rfloor T_{d}\right)+b_{\left\lfloor/ / T_{d}\right\rfloor\left\lfloor\tau_{2,1} / T_{d}\right\rfloor} \hat{R}_{a}\left(\tau_{2,1}-\left\lfloor\tau_{2,1} / T_{d}\right\rfloor T_{d}\right) \quad \text { (app.1) }
$$

where $\tau_{2,1}=\tau_{2}-\tau_{1}$. The first term in this equation represents the SI due to the previous symbol and the second term represents the interference due to the current symbol. Also, $R_{a}(\tau)$ and $\hat{R}_{a}(\tau)$ are the continuous-time partial cross-correlation functions of the time-delayed and reference sequences, and are given for $0 \leq \tau \leq T_{d}$ :

$$
\begin{aligned}
& R_{a}(\tau)=\int_{b}^{\tau} a(t-\tau) a(t) d t \\
& \hat{R}_{a}(\tau)=\int_{\tau}^{\tau_{d}} a(t-\tau) a(t) d t
\end{aligned}
$$

Theses integrals can be expressed in terms of the discrete aperiodic autocorrelation function $C_{a}(\zeta)$ of the spreading sequence and the continuous-time autocorrelation functions $R_{P_{T_{c}}}(\tau)$ and $\widehat{R}_{P_{T_{c}}}(\tau)$ of the chip waveform. The function $C_{a}(\zeta)$ is dependent upon the signature sequence and is given by:

$$
C_{a}(\zeta)=\left\{\begin{array}{cc}
\sum_{r=t^{\prime}}^{t^{\prime}+N_{d}-1+\zeta} a_{r} a_{r+\zeta} & 1-N_{d} \leq \zeta \leq 0 \\
\sum_{r=t^{\prime}}^{t^{\prime}+N_{r}-\zeta} a_{r+\zeta} a_{r+\zeta} & 0 \leq \zeta \leq N_{d}-1 \\
0 & |\zeta| \geq N_{d}
\end{array}\right.
$$

where $t^{\prime}=\left\lfloor t / T_{c}\right\rfloor$ 
The functions $R_{P_{T_{c}}}(\tau)$ and $\hat{R}_{P_{T_{c}}}(\tau)$ are the continuous-time partial autocorrelation functions of the binary spreading sequences, and are given for $0 \leq \tau \leq T_{c}$ :

$$
\begin{aligned}
& R_{P_{T_{c}}}(\tau)=\int_{b}^{\tau} P_{T_{c}}(t) P_{T_{c}}\left(t+T_{c}-\tau\right) d t \\
& \widehat{R}_{P_{T_{c}}}(\tau)=\int_{t}^{T_{c}} P_{T_{c}}(t) P_{T_{c}}(t-\tau) d t
\end{aligned}
$$

Through comprehensive analysis, the equation (app.1) can be written as [3]:

$W_{2,1}=w f\left(S_{2}\right)+z g\left(S_{2}\right)$

where $w, z$ are binomial distributions that are independent, and

$$
\begin{aligned}
& f\left(S_{2}\right)=\widehat{R}_{P_{T_{c}}}\left(S_{2}\right)+R_{P_{T_{c}}}\left(S_{2}\right) \\
& g\left(S_{2}\right)=\hat{R}_{P_{T_{c}}}\left(S_{2}\right)-R_{P_{T_{c}}}\left(S_{2}\right)
\end{aligned}
$$

For a rectangular chip waveform, $f\left(S_{2}\right)=T_{c}$ and $\left|g\left(S_{2}\right)\right| \leq T_{c} . \quad S_{2}=\tau_{2}-\tau_{1}-\gamma_{2} T_{c}$ and $\gamma_{2}=\left\lfloor\left(\tau_{2}-\tau_{1}\right) / T_{c}\right\rfloor$. Assuming $S_{2}$ is uniformly distributed on $\left(0, T_{c}\right)$, $\left|g\left(S_{2}\right)\right| \leq T_{c}$ is uniformly distributed in [-1, 1], seeing Fig. app.1.

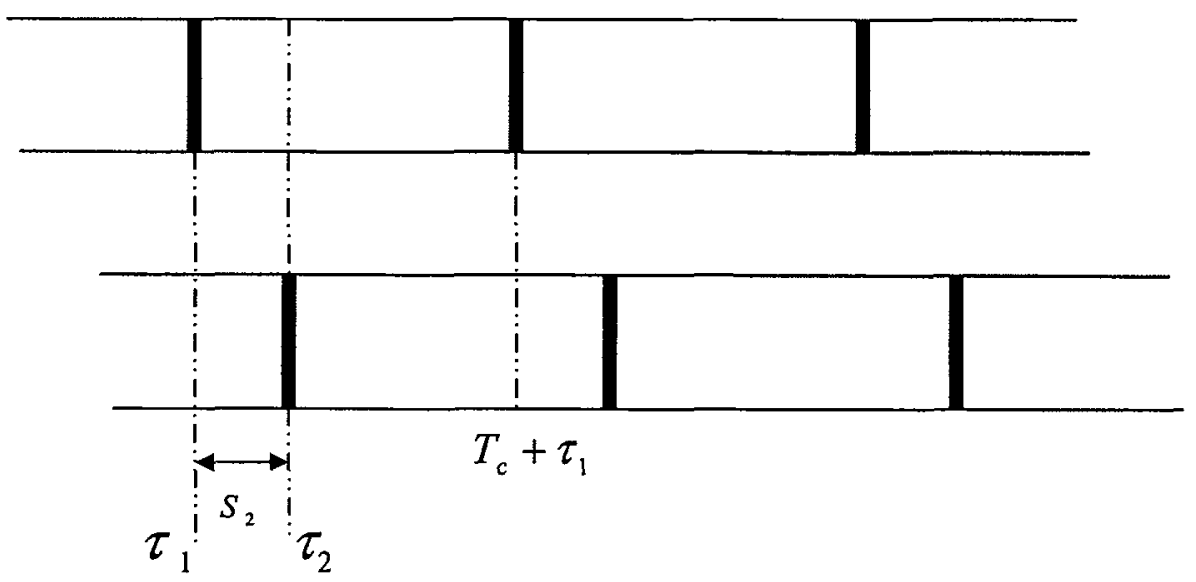

Fig.app.1: Time-delayed chip sequences that spread a data signal cause SI.

Therefore, for a rectangular chip waveform,

$$
W_{2,1}=w T_{c}+z g\left(S_{2}\right)
$$


$I_{2,1}=w+\frac{z g\left(S_{2}\right)}{T_{c}}$

Define $d=\frac{z g\left(S_{2}\right)}{T_{c}}$, then

$I_{2,1}=w+d$

Based on $z=0$ and $z \neq 0$ two cases exist leading to a PDF for $I_{2,1}$ conditioned on $C_{a}(1)=c$ :

$f_{I_{2,1}}(i \mid c)=f_{w}(i \mid c) * f_{d}(i \mid c)$

where "*" indicates convolution. $C_{a}(\zeta)$ is the discrete aperiodic autocorrelation function of the spreading signal $a(t)$, and

$$
C_{s}(1)=\sum_{r=0}^{N_{d}-1-1} a_{r} a_{r+1}=\sum_{r=0}^{N_{d}-2} c_{r}
$$

follows binomial distribution:

$$
f_{C_{o}(1)}(j)=\left\{\begin{array}{cc}
\left(\begin{array}{c}
N_{d}-1 \\
j+N_{d}-1 \\
2
\end{array}\right)\left(\frac{1}{2}\right)^{N_{d}-1} & j=1-N_{d,} 3-N_{d}, \cdots, N_{d}-3, N_{d}-1 \\
0 & \text { otherwise }
\end{array}\right.
$$

(app.12)

\section{The PDF of $H_{2,1}$ conditioned on $I_{2,1}$ when either uniformly distributed or a constant value}

Recall from equation (4.1.1) in Chapter 4, the SI term for a Rake receiver with one Rake finger and two MPGs:

$$
H_{2,1}=h_{2,1} \frac{\cos \theta_{2,1}}{T_{c}} W_{2,1}=\left|h_{1}\right|\left|h_{2}\right| I_{2,1} \cos \theta_{2,1}
$$

Assuming $I_{2,1}$ is uniformly distributed on an interval $(m, n)$ or a constant $k$ conditioned on $C_{a}(1)=c$, using the Jacobian, from the above equation, the PDF 
of $H_{2,1}$, i.e. the PDF of the SI for the cases when $I_{2,1}$ is either uniformly distributed or a constant $k$ can be written [3] as:

$$
\begin{aligned}
& f_{H_{2,1}}\left(x \mid i=\frac{(m+n)}{2} ; c\right)=\left\{\begin{array}{cc}
\frac{1}{\pi h_{2,1}(n-m)} \ln \left\{\frac{n}{m} \frac{\left(1+\sqrt{1-x^{2} /\left(n h_{2,1}\right)^{2}}\right)}{\left(1+\sqrt{1-x^{2} /\left(m h_{2,1}\right)^{2}}\right)}\right\} & 0 \leq|x| \leq m h_{2,1} \\
\frac{1}{\pi h_{2,1}(n-m)} \ln \left\{\frac{\left.n h_{2,1}\left(1+\sqrt{1-x^{2} /\left(n h_{2,1}\right)^{2}}\right)\right\}}{|x|}\right. & m h_{2,1} \leq|x| \leq n h_{2,1} \\
0 & |x| \geq n h_{2,1}
\end{array}\right. \\
& f_{H_{2,1}}(x \mid i=k ; c)=\left\{\begin{array}{cc}
\frac{1}{k \pi h_{2,1} \sqrt{1-x^{2} /\left(k h_{2,1}\right)^{2}}} & |x| \leq k h_{2,1} \\
0 & |x| \geq k h_{2,1}
\end{array}\right.
\end{aligned}
$$

Figure app.2 is a plot of this result, conditioned on $I_{2,1}$ being uniformly distributed $(5,7)$, denoted as $f_{I_{2,1}}(x \mid i ; c)$. From the curve of $f_{I I_{2,1}}(x \mid i ; c)$, it is easy to obtain the upper and lower bounds on the PDF of $H_{2.1}$ for each integer interval with the range of $x$, denoted by $u_{j}^{2,1}(i ; c)$ and $v_{j}^{2,1}(i ; c)$ respectively. The upper bound on the PDF over an integer interval corresponds to the maximum value of the PDF over that interval, while the lower bound on the PDF over an integer interval corresponds to the minimum value of the PDF over the interval.

Tighter bounds can be defined by further dividing each integer interval into smaller subintervals. If each discrete interval is divided into $N_{s}$ subintervals, the vectors $u_{j}^{2,1}(i ; c), i=-N_{d}, \cdots, N_{d}$ and $v_{j}^{2,1}(i ; c), i=-N_{d}, \cdots, N_{d}$, form the upper and lower bounds on the PDF of $H_{2,1}, i=-N_{d}, \cdots, N_{d}$, evaluated at $2 N_{d} N_{s}+1$ subintervals. 


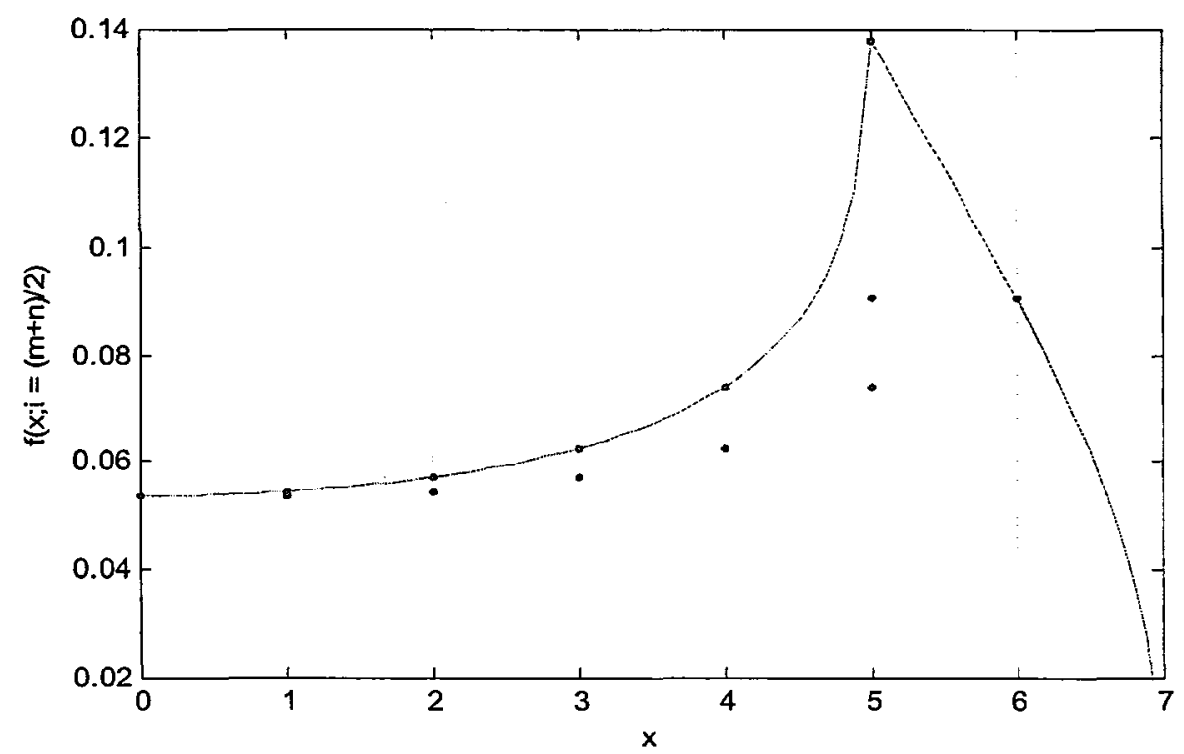

Fig.app.2: The PDF of $H_{2,1}$ conditioned on $I_{2,1}$ being uniformly distributed on (5, 7).

\section{The upper and lower bounds on the PDF of $H_{2,1}$}

Based on equations (app.10), (app.12) and (app.13) the upper and lower bounds on the PDF of $H_{2,1}$, i.e. the upper and lower bounds of SI for one rake finger with 2 MPGs, can be written as:

$$
\begin{aligned}
& u_{j}^{2,1}=\sum_{c=-N_{d} N_{s}}^{N_{d} N_{s}} u_{j}^{2,1}(c) f_{C_{a}(1)}(c) \\
& v_{j}^{2,1}=\sum_{c=-N_{d} N_{s}}^{N_{d} N_{s}} v_{j}^{2,1}(c) f_{C_{a}(1)}(c)
\end{aligned}
$$

where $u_{j}^{2,1}(c)=u_{j}^{2,1}(i ; c) f_{I_{2,1}}(i \mid c)$ and $v_{j}^{2,1}(c)=v_{j}^{2,1}(i ; c) f_{I_{2,1}}(i \mid c)$

If there are $\mathrm{K}>2$ received uncorrelated MPGs, the Rake decision variable will be:

$$
D_{1, \text { norm }}=N_{d} b\left|h_{1}\right|^{2}+\sum_{k=2}^{K} h_{k, 1} \frac{\cos \theta_{k, 1}}{T_{c}} W_{k, 1}+\frac{1}{T_{c}} \sqrt{\frac{T_{d}}{2 E_{b}}} \eta_{1}
$$


Due the fact that the cross correlations between each time-delayed sequence and the reference sequence are independent, $u_{j}^{2.1}, u_{j}^{3.1}, u_{j}^{4,1}, \ldots, u_{j}^{K, 1}$ are independent, also $v_{j}^{2,1}, v_{j}^{3,1}, v_{j}^{4,1}, \ldots, v_{j}^{K, 1}$ are independent. Thus, the upper and lower bounds on the PDF of SI due to the K-1 interferences for a correlation receiver can be written as:

$$
\begin{aligned}
& u_{j}=u_{j}^{2,1} * u_{j}^{3,1} * u_{j}^{4,1} * \ldots * u_{j}^{K, 1} \\
& v_{j}=v_{j}^{2,1} * v_{j}^{3,1} * v_{j}^{4,1} * \ldots * v_{j}^{K, 1}
\end{aligned}
$$

In a similar development, one can obtain the upper and lower bounds on the PDF of SI for a rake receiver with $L$ rake fingers.

\section{Upper and lower bounds of BER}

In the condition when SI is constant, there is only one random variable $\eta_{\text {Rake }}$ in equation (4.1.1), which is Gaussian with zero mean and variance $\sigma_{\text {Rake }}^{2}$. The BER for a Rake receiver with L Rake fingers in non-fading channel can be written in terms of the Gaussian $Q(\cdot)$ function, as:

$$
\begin{aligned}
& P_{E}\left(N_{d} \sum_{i=1}^{L}\left|h_{i}(n)\right|^{2}, \beta\right)=Q\left(\frac{\left(N_{d} \sum_{i=1}^{L}\left|h_{i}(n)\right|^{2}+\beta\right)}{\sigma_{\text {Rake }}}\right) \\
& =\frac{1}{\sqrt{2 \pi}} \int_{\frac{N_{d} \sum_{i=1}^{L}\left|h_{i}(n)\right|^{2}+\beta}{\sigma_{\text {Rake }}}}^{\infty} e^{-\frac{w^{2}}{2} d w}
\end{aligned}
$$

where $\beta$ is constant SI, $\sigma_{\text {Rake }}^{2}=\frac{N_{d}^{2} N_{0} \sum_{i=1}^{L}\left|h_{i}(n)\right|^{2}}{2 E_{b}}$.

On a fading channel, SI is a continuous random variable. In order to accurately 
model this interference, the upper and lower bounds on the PDF of SI can be used. The PDF of SI is nonzero only on an interval $[-L(K-1), L(K-1)]$. Considering normalized version of this PDF and partition of the interval $\left[-N_{d} L(K-1), N_{d} L(K-1)\right]$ into a number, $N_{s}$ of subintervals, there are total $2 L(K-1) N_{d} N_{s}$ subintervals.

To obtain bounds on the average probability of error of the $\mathrm{Rx}$, one can assume that with probability $u_{j}$ the SI is equal to the value in the interval $\left[j N_{s}^{-1},(j+1) N_{s}^{-1}\right]$ that yields the largest probability of error. Since the function $Q(\cdot)$ is a strictly decreasing function, for the correlation receiver, this value is $j N_{s}^{-1}$. Then the upper bound on BER performance at time instant $\mathrm{n}$ can be written as:

$$
p_{E}^{U}(n)=\sum_{j=-L(K-1) N_{d} N_{s}}^{L(K-1) N_{d} N_{s}} u_{j} P_{E}\left(N_{d} \sum_{i=1}^{L}\left|h_{i}(n)\right|^{2}, \frac{j}{N_{s}}\right)
$$

and the lower bound on BER performance at time instant time $\mathrm{n}$ is given by:

$$
p_{E}^{L}(n)=\sum_{j=-L(K-1) N_{d} N_{s}}^{L(K-1) N_{d} N_{s}} v_{j} P_{E}\left(N_{d} \sum_{i=1}^{L}\left|h_{i}(n)\right|^{2}, \frac{j+1}{N_{s}}\right)
$$

where $h_{i}(n)$ represents the amplitude of the MPG corresponding to time delay $\tau_{i}$ from the $n^{\text {th }}$ set of MPGs in a time series of SREs.

Then the average upper and lower bounds on BER can be written as:

$$
\bar{p}_{E}^{U}=\frac{1}{N} \sum_{n=1}^{N} P_{E}^{U}(n), \bar{P}_{E}^{L}=\frac{1}{N} \sum_{n=1}^{N} P_{E}^{L}(n)
$$

